\title{
The evolution of foresight: What is mental time travel, and is it unique to
} humans?

\author{
Thomas Suddendorf \\ School of Psychology, University of Queensland, Brisbane, Queensland 4072, \\ Australia \\ t.suddendorf@psy.uq.edu.au \\ Michael C. Corballis \\ Department of Psychology, University of Auckland, Private Bag 92019, \\ Auckland 1142, New Zealand \\ m.corballis@auckland.ac.nz
}

\begin{abstract}
In a dynamic world, mechanisms allowing prediction of future situations can provide a selective advantage. We suggest that memory systems differ in the degree of flexibility they offer for anticipatory behavior and put forward a corresponding taxonomy of prospection. The adaptive advantage of any memory system can only lie in what it contributes for future survival. The most flexible is episodic memory, which we suggest is part of a more general faculty of mental time travel that allows us not only to go back in time, but also to foresee, plan, and shape virtually any specific future event. We review comparative studies and find that, in spite of increased research in the area, there is as yet no convincing evidence for mental time travel in nonhuman animals. We submit that mental time travel is not an encapsulated cognitive system, but instead comprises several subsidiary mechanisms. A theater metaphor serves as an analogy for the kind of mechanisms required for effective mental time travel. We propose that future research should consider these mechanisms in addition to direct evidence of future-directed action. We maintain that the emergence of mental time travel in evolution was a crucial step towards our current success.
\end{abstract}

Keywords: animal cognition; cognitive evolution; comparative psychology; episodic memory; memory systems; mental time travel; planning; prospection

He said "What's time? Now is for dogs and apes! Man has Forever!"

— Robert Browning, (1896) A Grammarian's Funeral, p. 425.

\section{Introduction}

Time travel may never be physically possible (Holden 2005). For now at least, humans can travel in time only in their minds. Mental time travel is a term we coined to refer to the faculty that allows humans to mentally project themselves backwards in time to re-live, or forwards to pre-live, events (Suddendorf \& Corballis 1997). Past and future travels share phenomenological characteristics and activate similar parts of the brain. Mentally reliving past events is also known as episodic memory in the literature and has been the topic of intense research efforts (e.g., Tulving 1984; 2005). By contrast, mental construction of potential future episodes has only very recently begun to draw attention. Nevertheless, there is growing recognition that mental time travel into the past and future are related, and that the ultimate evolutionary advantage must lie with the capacity to access the future (Dudai \& Carruthers 2005a; Suddendorf \& Busby 2003b; 2005; Suddendorf \& Corballis 1997; Tulving 2005). Though we may often get it wrong, humans have in general been extraordinarily successful in foreseeing, planning, and shaping the future, and indeed allowing us to influence the earth itself in extraordinary but not always benevolent ways (Dawkins 2000).

Since present behavior can increase or decrease an individual's future survival chances, one might expect many species to have evolved anticipatory capacities. The world is dynamic, and organisms that can pick up on significant regularities (e.g., fluctuations in food availability) and act in tune with them (e.g., being in the right place at the right time) have an advantage over those that do not. Many organisms actively influence their own futures by creating an environment that suits their needs (socalled niche-construction, Odling-Smee et al. 2003), such as when a beaver dams a stream. However, futureoriented mechanisms vary in flexibility, and relatively inflexible mechanisms will often suffice.

Through natural selection, some species have evolved behavioral predispositions to exploit significant long-term regularities (e.g., seasonal variations). A hibernator, for example, may hoard food for an impending winter even if individually it has never experienced a winter. Such instinctual future-directed behavior serves well, as long as the environmental pattern persists. But even longterm regularities may at times change drastically (e.g., climate change), and organisms fixed to a pattern that no 
longer prevails are disadvantaged relative to those that have more flexibility. This can be achieved by individual fine-tuning mechanisms, such as critical periods for parameter setting, imprinting, and other forms of learning. Indeed, learning and memory in general may be regarded as future-oriented adaptations that allow an individual, rather than a population, to adjust to local change and track short-term regularities.

Here we propose a taxonomy of how memory systems differ in what they provide for the future. We argue that mental time travel is the most flexible of those memorybased systems, and the most recently evolved. We then review evidence for mental time travel in nonhuman animals, and suggest a framework that identifies subsidiary mechanisms of mental time travel that nonhuman species may or may not possess.

First, though, it is useful to distinguish perceptual systems that detect and track relevant information, from action systems that control behavior itself. Many animal species, as well as human neurological patients and children, show dissociations between what they know in the perceptual domain and what knowledge they can use to control action (Hauser 2003; Sterelny 2003). Perceptual systems differ in the robustness of their tracking (e.g., use of single versus multiple perceptual channels to track significant aspects of the environment) and storage of information (e.g., knowing what is currently where).

Thomas Suddendorf was born and raised in Germany but has spent most of his adult life further South. He completed both his Masters (1994) and Ph.D. (1998) theses under supervision of Michael Corballis in New Zealand, before taking up a position at The University of Queensland, Australia, where he is currently Associate Professor of Psychology. He has published extensively on the evolution of mental time travel and the representational capacities of apes and young children. His work has attracted several recognitions, including most recently the Early Career Award of the Academy of the Social Sciences in Australia and the Frank A. Beach Award of the American Psychological Association Division 6 (Behavioral Neuroscience and Comparative Psychology).

Michael Corballis received his Ph.D. from McGill University in 1965, and served on the faculty there until 1977, when he took up his present position as Professor of Psychology at the University of Auckland. He has published on many aspects of cognitive neuroscience, including cerebral asymmetry, memory, imagery, and the evolution of language. He is a Fellow of the AAAS, the American Psychological Association, the American Psychological Society, and the Royal Society of New Zealand, and in 2002 was appointed Officer of the New Zealand Order of Merit (ONZM) for services to psychological science. He was recently elected President of the International Neuropsychological Society.
Action systems differ in the flexibility or response breadth they provide (Sterelny 2003); for example, they may include relatively narrow options such as hibernating or storing food, or highly flexible options like cooking and preserving food in diverse ways. Organisms may have sophisticated mechanisms to track temporal information and yet inflexible action systems, and vice versa. Further, the link between the two can be direct, or bottom-up, when perception of a stimulus triggers a response, or they can be top-down, mediated by internal representations (i.e., declarative memory). Top-down mediation offers the opportunity for representations decoupled from the immediate input to drive action flexibly and independently.

\section{A taxonomy of future-oriented cognition}

Figure 1 shows a widely accepted taxonomy of human memory systems (e.g., Miyashita 2004; Squire 1992), and illustrates how this can serve as a basis for a parallel taxonomy of adaptation to the future.

Non-declarative or implicit memory systems are so called because, in humans, their content cannot be declared or verbalized (Tulving 1985). They allow stimulus-driven prediction of regularities. For example, through association, a conditioned stimulus (e.g., a sound) predicts the future arrival of an unconditioned stimulus (e.g., food) and triggers a future-directed response (e.g., salivation). In operant conditioning a behavioral response predicts a certain outcome (reward). Learning theory has described how organisms use associations to predict the near future, and a growing literature is mapping its neurophysiological basis (e.g., O’Doherty 2004; Schultz 2006). Non-associative changes in behavior, such as habituation, also can be understood in terms of expectations (e.g., that the situation stays unchanged). All these non-declarative memory systems allow behavior to be modulated by experience such that the organism gains a future advantage. We may call the resultant future-directed mechanisms "procedural" because flexibility extends only to learning to respond to current indicators of upcoming events. The behavior is stimulus-bound, or better, bound to the perceptual tracking of stimuli.

Declarative or explicit memories provide greater flexibility because they can also be voluntarily triggered topdown from the frontal lobes, rather than bottom-up through perception (Miyashita 2004). They may be regarded as decoupled representations that are no longer directly tied to the perceptual system. In humans, these memories are conscious and at least partly verbalizable (Tulving 1985; 2005). Declarative memory can be subdivided into semantic memory and episodic memory. Semantic memory contains general knowledge, allowing learning in one context to be voluntarily transferred to another. This capacity provides the basis for inferential and analogical reasoning. Semantic memory may thus enable semantic prospection that is voluntary and not stimulus-bound. Nevertheless, such prospection is restricted in that it builds on a knowledge base that is impervious to particularities of the learning event itself. It is the second component of declarative memory, namely, episodic memory, that gives rise to the notion of mental time travel. 


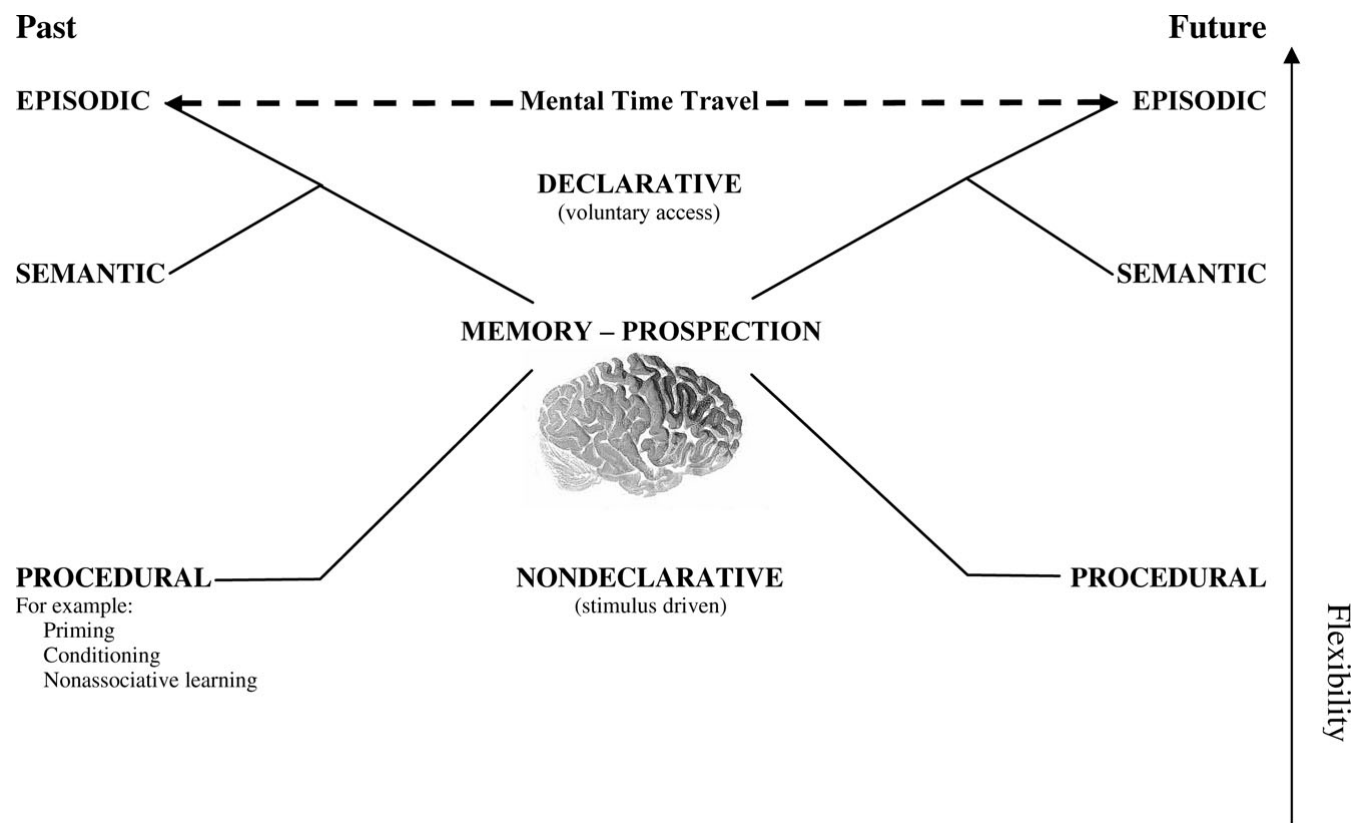

Figure 1. Memory and prospection systems. The common taxonomy of memory systems (left), after Squire (1992), and its proposed prospective counterpart (right).

\subsection{Toward a definition of mental time travel}

Episodic memory, in contrast to semantic memory, provides access to the personally experienced event, rather than just the knowledge extracted from the event. The phenomenological experience of remembering, or what Tulving (1985) calls autonoetic or self-knowing consciousness, such as recollecting where and when one learned that Wellington is the capital of New Zealand, is different from merely knowing that fact. This distinction is supported by experimental manipulations and imaging studies, and by dissociations in impairments following brain injury (Gardiner et al. 2002; Henson et al. 1999; Klein et al. 2002b; Tulving 2005). Thus, amnesic patients, such as K.C., may know facts (e.g., the difference between stalagmites and stalactites) and procedures (e.g., how to play chess), without being able to recall a single personally experienced event leading to knowing them (Tulving 2005). Episodic memory is not about regularities, but about reconstructing particularities of specific events that have happened to the individual.

In effect, then, episodic memory implies a mental reconstruction of some earlier event, including at least some of the particularities of that event, such as the principal characters involved, the actions that took place, the setting, and the emotional reactions. Metaphorically speaking, it might be regarded as the result of a mental journey into the past. This idea is readily extended to the future. Based on previous experiences, we can imagine specific events in the future, including the sorts of particularities that have characterized events in the past. Mental time travel into the future might include the planning of some specific event, such as a dinner party, or it might involve the mental anticipation of some event that we know to be scheduled for some future date, such as a job interview. Again, though, there is a distinction between merely knowing that some event will occur, such as that the sun will set, and mentally creating an event, such as a sunset actually experienced, with gradual fading of light, and the blue flash on the horizon as the last image of the sun disappears.

The mental reconstruction of past events and construction of future ones may have been responsible for the concept of time itself, and the understanding of a continuity between past and future. Having a concept of time allows us to understand that past and future are on the same dimension, and what was the future eventually becomes the past (that is, unless the universe comes to an end). Mental time travel allows us to imagine events at different points along this continuum, even at points prior to birth or after death. This means that mental time travel is a generative process, incorporating known elements but arranged in particular ways to create the experience of events that are actually occurring. Even episodic memory may not be a faithful recreation of a past event. False memories have been widely documented (e.g., Loftus \& Ketcham 1994) and are readily created in the laboratory (e.g., Roediger \& McDermott 1995). This means that mental time travel cannot be defined in terms of the veracity of the content. We know what mental time travel is because we can introspectively observe ourselves doing it and because people spend so much time talking about their recollections and anticipations.

A major challenge, though, is to establish a definition, or set of criteria, that might identify mental time travel in nonhuman animals that cannot express their experiences in words. This problem is akin to the decades-long search for behavioral criteria for theory of mind (see sect. 4.3) in nonhuman animals (Heyes 1998; Povinelli \& Vonk 2003; Premack \& Woodruff 1978; Suddendorf \& Whiten 2003; Tomasello et al. 2005; Whiten \& Byrne 1988). Tulving (1972) originally defined episodic memory in terms of the kind of information it appears to store: namely, what happened where and when. This is 
the so-called www criterion; that is, demonstrated understanding of what, where, and when might be considered sufficient evidence that an imagined event is an instance of mental time travel. For example, if an animal that caches food can be considered to demonstrate understanding of what was cached, where it was cached, and when it was cached, then it has episodic memory for the caching event itself. Similarly, if its behavior indicates specific knowledge of a future event, such as what food it will retrieve, where it will find it, and when it will retrieve it, then it may be said to have travelled mentally into the future to a retrieval event itself. However, although useful, the www criterion is neither necessary nor sufficient for mental time travel. Personal experience, as well as research on false memories, show that one can mentally revisit an event without accurate information as to what, where, and when, and conversely one can know what, where, and when something happened (e.g., one's birth) without remembering the event itself (Suddendorf \& Busby 2003b). Thus, Tulving changed his definition to emphasize the phenomenological experience (autonoetic consciousness) of episodic retrieval (Tulving 1985; 2005), and www memory in animals has been more cautiously termed "episodic-like memory" (Clayton \& Dickinson 1998).

Phenomenological experience, though, is a private mental capacity, and one may rightfully wonder if one could ever know of another organism's mental world. It is conceivable that neurophysiological criteria may eventually suffice, but there is as yet no agreement as to the conditions defining phenomenological awareness, even in humans. Nevertheless, for mental time travel to have evolved, there must have been something natural selection could work on, that is, some effect on survival or reproduction (unless it was merely an accidental side effect of some other adaptation). The answer we propose is that mental time travel provides increased behavioral flexibility to act in the present to increase future survival chances (Suddendorf \& Busby 2003b; 2005). For example, one might prepare for a forthcoming job interview based on past experiences of interviews, and on imagining the questions one may be asked and on one's possible replies to them.

If this argument is correct, then the crux of mental time travel lies in its role in enhancing biological fitness in the future, so that mental time travel into the past is subsidiary to our ability to imagine future scenarios. Episodic memory may also inform semantic prospection by, for example, providing boundary conditions for the scope of generalizations. Indeed, memories for particular contradicting episodes are primed when people retrieve semantic generalizations (Klein et al. 2002a). But we argue that the primary role of mental time travel into the past is to provide raw material from which to construct and imagine possible futures. In establishing whether a given behavior is indicative of mental time travel, then, we should consider application of that behavior to the future rather than to the past (Suddendorf \& Busby 2005). We therefore advance the following description of conditions that, if met, may entitle us to invoke the construct of mental time travel to explain it.

Mental time travel is evident in voluntary behavior that solves a problem that the organism will encounter at a future point in time, where "future" entails that the problem is not already manifest (as when acting to satisfy a current hunger, for example). To ascertain that a given behavior was driven by mental time travel, it is necessary to rule out chance, innate predispositions, procedural and semantic prospection, or any combination of these. We postulate that the crucial selective advantage mental time travel provides is flexibility in novel situations and the versatility to develop and adopt strategic longterm plans to suit individual selected goals. Thus, paradigms that use transfer tests and cross different domains would be strongest in making cases for mental time travel. Such tests provide evidence for mental time travel into the past only by virtue of the evidence linking past and future mental time travel, to which we turn next.

\subsection{Evidence for continuity of past and future mental time travel}

Since past is fact and future is fiction, common sense might suggest that different cognitive mechanisms underlie recollection of past events and construction of future ones. There is a fundamental causal asymmetry, and one simply cannot know the future as one knows the past. However, various lines of evidence suggest that mental time travel into the past shares cognitive resources with mental construction of potential future episodes (Suddendorf \& Corballis 1997). Normal adults report a decrease in phenomenological richness of both past and future episodes with increased distance from the present (D’Argembeau \& Van der Linden 2004). The temporal distribution of past events people envisage follows the same power function as the temporal distribution of anticipated future events (Spreng \& Levine 2006). Amnesic patients who are unable to answer simple questions about yesterday's events have been found to be equally unable to say what might happen tomorrow (Klein et al. 2002b; Tulving 1985), and it is not until around age 4 that children are able to accurately answer both such questions (Busby \& Suddendorf 2005). Patients with depression who have trouble retrieving specific memories from their past also have trouble imagining specific future episodes (Williams et al. 1996). Finally, brain imaging has shown that both remembering the past and imagining the future are associated with frontal and temporal lobe activity, although there are specific areas in the frontal pole and medial temporal lobes that are more involved with the future than with the past (Okuda et al. 2003).

The constructions of future and past episodes both depend in part on semantic memory, since one must construct events that are consistent with one's general knowledge of the world. Nevertheless, it is the episodic component that provides the particularities that can fit one's future plans precisely to the occasion - the seating arrangement, the clothes one might wear, the menu, the topics of conversation. In allowing us to foresee unique events, mental time travel offers the ultimate step in adaptation to the future. Like human language, it is openended and generative, so there is no end to the number of potential future scenarios one might envisage.

Although episodic memory preserves something of the particularities of individual events, it is often unreliable and subject to distortion, as we have seen. In cases of amnesia, moreover, it is episodic memories that are the most vulnerable (Wheeler et al. 1997). The fact that episodic memory is fragmentary and fragile suggests that 
its adaptiveness may derive less from its role as an accurate record of personal history than from providing a "vocabulary" from which to construct planned future events (and perhaps to embellish events of the past). It may be part of a more general toolbox that allowed us to escape from the present and develop foresight (Suddendorf \& Corballis 1997), and perhaps create a sense of personal identity (Schacter 1996). Indeed, our ability to revisit the past may be only a design feature of our ability to conceive of the future (Suddendorf \& Busby 2003b).

\subsection{Neurophysiological evidence}

We have suggested that neurophysiological evidence may eventually serve as a proxy for the phenomenology of mental time travel. There is increasing evidence concerning the neurophysiology of mental time travel in humans, but little from nonhuman animals. Some of this evidence suggests, in fact, that mental time travel may be uniquely human, since the brain areas involved have apparently undergone changes that are not evident in other primates. For example, the key to mental time travel may lie in part in the expansion of the brain, and the prefrontal cortex in particular, in human evolution. Brain size varies between species partly as a function of body size, and an appropriate comparative measure is the encephalization quotient (EQ), devised by Jerison (1973), which is based on the regression of brain weight on body weight. The human $\mathrm{EQ}$ is about three times that of the chimpanzee. Further, it has been claimed that the increase is larger in the prefrontal cortex, known to be critically involved in episodic memory (see review by Wheeler et al. 1997) than in other brain areas (Deacon 1997). Although this disproportionate increase has been disputed (e.g., Semendeferi et al. 1997; Uylings 1990), Deacon (1997) argues that other studies have failed to measure prefrontal cortex independently of motor and premotor areas. A more recent study confirms the disproportionate enlargement of the prefrontal cortex in humans, but indicates that it is restricted to white matter, and does not apply to grey matter (Schoenemann et al. 2005).

Regardless of the question of size, there is also evidence that the prefrontal cortex has been substantially reorganized in the course of human evolution. For example, Area 13, which appears to be a subdivision of Area 11, is only about half that expected on the basis of brain size, and Semendeferi et al. (1998) suggest that this diminution occurs because other regions of Area 11, along with regions of Area 47, have been enlarged to accommodate a large number of specialized subdivisions. The frontal pole is also considerably enlarged relative to that in apes, especially in the right hemisphere (Semendeferi et al. 2001). Summarizing this evidence, Flinn et al. (2005) suggest that these changes to Area 11 and the right prefrontal cortex appear to be involved in "self awareness, social problem solving, the ability to recall personal experiences, and the ability to project oneself into the future" (pp. 30-31).

The involvement of the prefrontal cortex may depend on neural loops that also encompass the basal ganglia and perhaps other regions as well. In one functional magnetic resonance imaging (fMRI) study, for example, brain activity was recorded while human subjects learned actions in a Markov decision task that would bring either immediate or future rewards (Tanaka et al. 2004). In both cases significant activity was seen in the lateral orbitofrontal cortex and striatum, but for actions concerning future rewards, there was additional activation in the dorsolateral prefrontal cortex, inferior parietal cortex, dorsal raphe nucleus, and cerebellum. Regression analysis also suggested graded maps of time scale within the insula and striatum, with ventroanterior regions involved in predicting immediate rewards and dorsoposterior regions in predicting future rewards. In another study involving appetitive conditioning in humans, fMRI recordings showed activity in the orbitofrontal cortex and striatum to be dependent on the time between a present conditioned stimulus and an anticipated reward (O'Doherty et al. 2002; see also O’Doherty 2004).

\section{Mental time travel in nonhuman animals?}

Nonhuman species commonly display behaviors that depend on non-declarative future-oriented systems, and there is strong evidence that some of them can also deploy semantic memory for future-directed action. For example, the chimpanzee Panzee declared where food was hidden outside her enclosure by touching a lexigram denoting the food and directing a naïve human to it by pointing to its location (Menzel 2005). Although this shows that she knew where the food was, it does not prove that she remembered the hiding event itself, just as one can know where the car keys are without remembering the event of putting them there. The "linguistic" outputs of trained apes may demonstrate declarative (i.e., semantic) memory, such as the knowledge of which symbols go with which objects or actions, but they do not include reports of travels down memory lane. They have not provided evidence of mental time travel. There is so far no use of tense, nor any sense that the animals are telling stories about previous or anticipated episodes. In marked contrast, human conversation is replete with references to past and planned future episodes (e.g., Szagun 1978).

\subsection{Mental time travel into the past}

Despite our earlier reservations, the www criterion for episodic memory has featured prominently in recent comparative research. Though one might expect to find precursors of mental time travel in our closest living relatives, the most vigorously argued case has come, not from apes, but from birds that cache food in various locations and later retrieve it. Scrub jays can select food locations not only according to the type of food, but also according to how long it has been stored. For example, they will recover recently cached worms in preference to nuts, since fresh worms are more palatable; but if the worms have been cached for too long, they will retrieve nuts, because the worms will have decayed and become unpalatable (Clayton et al. 2003). Scrub jays seem to know what has been cached, where it was cached, and when it was cached, thus apparently meeting the www criterion. As noted earlier, however, investigators have used the term "episodic-like memory" in acknowledgment that the www criterion need not imply true episodic memory, in part because there need not be any implications 
about autonoetic consciousness (Clayton et al. 2003; Emery \& Clayton 2004).

When humans talk about past events, they typically exchange information about even more "w"s, such as "who did what to whom, and when, and where, and why, and what happened next" (Suddendorf \& Corballis 1997, p. 159). Recent evidence indicates that jays may indeed also store information about who observes them cache (Dally et al. 2006b). The birds were more likely to move the food to new locations if a more dominant bird observed the caching than if a less dominant bird did so. The outcome of this re-caching behavior was presumably a reduction in the likelihood of the food being pilfered in future.

This innovative research program on scrub jays has inspired similar work on other species, with varying success (see Table 1 for a summary). Rhesus monkeys, for example, seemed unable to learn that a preferred food was available only after a short interval, but not after a long one (Hampton et al. 2005), whereas rats were able to learn that a particular arm of a maze contained chocolate pellets after a long delay but not after a short delay (Babb \& Crystal 2005).

The cases listed in Table 1 explicitly link their results to one of the "w"s of episodic-like memory. Numerous earlier studies might be included when re-categorized in line with this interpretation (see, e.g., Schwartz \& Evans 2001). No doubt this table will be expanded and altered further as more comparative research is conducted with the explicit aim of uncovering evidence for episodic-like memory. We believe that this approach may provide one of the first areas in which research systematically maps the types of mental content in different species. Although we look forward to a more comprehensive table, we remain unconvinced that any of these cases shows true mental time travel (Hampton \& Schwartz 2004; Roberts 2002; Suddendorf \& Busby 2003b).

Suddendorf and Busby (2003b) put forward various reasons why the data from jays and other species are not yet conclusive. In the present article, we have noted earlier the distinction between the type of information stored and the experience of mentally revisiting an episode that led Tulving to change his definition of episodic memory. The information listed in Table 1 may be known rather than remembered. There is a difference between remembering having put something somewhere, some time ago, and simply knowing right now what is where, and how fresh it is. In fact, episodic-like memory may result from feed-forward mechanisms that need not be about the past at all. Episode A may cause cognitive change $\mathrm{B}$, which may in turn affect later behavior $\mathrm{C}$, without B carrying any information about A itself (Dretske 1982). For example, performance that depends on time since an event may rely on the strengths of memory traces, which fade in time, and may therefore provide a direct cue without implying mental travel through time. Trace strength or some other timedependent process may act as a clock to determine a "use-by" date for consumption.

One argument against this specific idea that www memory may depend on trace strength is that rats, at least, still show the effects of trace strength in the accuracy of recognition after hippocampal lesions, yet lose the ability to discriminate temporal order (Eichenbaum et al. 2005), a finding that is also consistent with the hippocampus being a primary site for episodic memory in humans. Even so, the hippocampus may be involved in access to trace strength as a discriminative cue, so failure to make temporal discriminations after hippocampal damage need not rule out the trace-strength hypothesis. Whether or not this specific hypothesis is correct, it should be clear that there are viable alternatives to the notion that evidence for www, or even wwww, memories is evidence for mental time travel. Indeed, even the original proponents of this approach concede that these memories may exist without the jays mentally reconstructing the past (Dally et al. 2006b).

A few researchers have tried to tackle the question with different sets of innovative methodologies (Zentall 2006). For example Zentall et al. (2001) taught pigeons to press one or the other of two new keys depending on whether they had previously responded or not responded on another earlier key - which suggests an ability to refer to a previous behavioral episode. Similarly, dolphins appear to remember their own previous behavior, as shown by an ability to respond to commands equivalent to sentences like "do something not recently done," or "repeat the most recent response" (Mercado et al. 1998). Rats show evidence for temporal order. When exposed to sequences of four different odors in distinct locations, rats later

Table 1. Recent studies suggesting knowledge of different "w" information in various species that form part of "episodic-like" memory

\begin{tabular}{|c|c|c|c|c|c|}
\hline Species & What & Where & When & Who & Example Studies \\
\hline Chimpanzees & Yes & Yes & $?$ & Yes & (Menzel 2005) \\
\hline Crested Gibbons & Yes & Yes & $?$ & $?$ & (Scheumann \& Call 2006) \\
\hline Gorillas & Yes & Yes & $?$ & Yes & $\begin{array}{l}\text { (Schwartz et al. 2002; Schwartz et al. } \\
\text { 2005) }\end{array}$ \\
\hline Hummingbirds & $?$ & Yes & Yes & $?$ & (Henderson et al. 2006) \\
\hline Mice & Yes & Yes & Yes & $?$ & (Dere et al. 2005) \\
\hline Orangutans & Yes & Yes & $?$ & $?$ & (Scheumann \& Call 2006) \\
\hline Rats & Yes & Yes & Yes & $?$ & $\begin{array}{l}\text { (Babb \& Crystal 2005; Eacott et al. } \\
\text { 2005) }\end{array}$ \\
\hline Rhesus monkeys & Yes & Yes & No & $?$ & (Hampton et al. 2005) \\
\hline Scrub jays & Yes & Yes & Yes & Yes & $\begin{array}{l}\text { (Clayton \& Dickinson 1998; Clayton } \\
\text { et al. 2001; Dally et al. 2006b) }\end{array}$ \\
\hline
\end{tabular}


showed above-chance performance on tests in which they were to choose which of two odors or locations had occurred earlier in the sequence (Eichenbaum et al. 2005). However, all of these approaches run into problems of interpretation similar to those that afflict the "www" approach. As we suggested earlier here and elsewhere (Suddendorf \& Busby 2005), the solution may be to focus on future-directed behavior instead of on episodic memory. We argue that it is action with the future in mind that provides increased selective advantage and is hence visible to evolution. If we are right, it might also be visible to clever researchers (if nonhuman animals have it).

\subsection{Mental time travel into the future}

Animals meet significant recurrent problems such as seasonal food shortages in different ways (e.g., through migration, torpor, increased fat storage). Caching food is a solution that has evolved among some mammals and birds that provide for their young (Smith \& Reichman 1984). This strategy rests on some memory capacities and suggests orientation to future consumption, but it need not imply that the animal actually envisages that future, or even explicitly plans for it. For example, one study showed that rats continued to cache food in locations in a maze where food was later repeatedly degraded or pilfered, even though they avoided those locations in retrieving the food (McKenzie et al. 2005). This suggests that they had little sense of what would happen in the future. Similarly, young scrub jays vigorously cache all kinds of objects well before they develop the behavior of retrieving them. When caching and retrieval develop in such predictable fashion, it appears to be based on instinctive or implicitly learned predispositions that may be modulated by semantic memory but need not involve mental time travel (Suddendorf \& Busby 2003b).

A possible test of "episodic-like" prospection might run as follows. The birds would first be taught that if they select a cache location under one condition (say, a green light) they will be allowed to retrieve from that location at any time, but if they select under another condition (say, a red light) they must wait at least a day before being allowed to retrieve. Assuming that they could learn this, they are then given the choice of worms or nuts, and the question is whether they would select fresh worms when allowed immediate access, but would anticipate decayed worms when access is delayed, and so choose nuts. If such anticipation were possible, it might indicate an understanding that how long food has been cached projects into the future as well as into the past. However, the same difficulties as with "episodic-like" memory apply. The birds might seem to understand the state of the world tomorrow (rotten worms) only through a combination of predispositions and specific learning algorithms that have evolved in the caching context (Dally et al. 2006b; Suddendorf \& Busby 2003b).

Whatever the outcome of such an experiment, adult jays have been shown to modulate caching behavior in ways that seem to take the possibility of future pilfering into account. Earlier, we alluded to evidence that they recache food if observed by a more dominant bird (Dally et al. 2006b). Another study has shown that, when observed caching food, jays re-cache food only if they themselves had previously stolen the food of others. Birds with experience of theft seem to anticipate a future in which their own food might be stolen, and act accordingly (Emery \& Clayton 2001). It takes a thief to know a thief.

Prevention of pilfering is a common adaptive problem for species that store food, and various strategies have evolved to deal with it (for a review, see Dally et al. 2006a). Both scatter-hoarders (e.g., ravens) and larderhoarders (e.g., acorn woodpeckers) may carefully disguise their caches or actively defend their stores (e.g., Bugnyar \& Heinrich 2006). Strategies of pilferage avoidance are increasingly being documented. For example, Eastern grey squirrels space caches further apart when conspecifics are present and preferentially dig oriented away from them (Leaver et al., in press). Theft is not the only future problem that hoarders may address. Gray squirrels bite out the seeds of white oak (but not red oak) acorns before storage, which prevents germination (Steele et al. 2001). Treating food before storage (e.g., pine squirrels dry mushrooms in the sun) or keeping it alive but unable to escape (e.g. moles biting worms) are strategies that reduce spoilage and hence increase future survival chances (e.g., Smith \& Reichman 1984).

Are these future-oriented behaviors fundamentally different from non-behavioral species-specific adaptations to the same problem, such as seasonally storing food as blubber or body fat? Perhaps not. Advantageous behavior can lead to evolutionary change (Bateson 2004) and can become instinctive as descendants of animals displaying the behavior face selection pressure for reliable, less resource-intensive shortcuts. New Caledonian crows, for example, manufacture and use tools (Hunt 1996). Although there is evidence for social transmission (Hunt \& Gray 2003), the birds appear to have inherited a predisposition for tool use. They show such behavior without any experience or opportunity for observation (Kenward et al. 2005). Adaptations for certain types of memory and learning have also been reported in regent honeyeaters. In line with ecological factors (i.e., replenishment rates), these birds easily learn to avoid recently rewarding food locations and to return after long periods, but do poorly when the contingency is the other way around (Burke \& Fulham 2003). Though this learning is appropriately future-oriented to the contingencies of its habitat, such constraints distinguish it from the flexible future-oriented behavior characteristic of human mental time travel.

\subsection{The Bischof-Köhler hypothesis}

We argued earlier that the selective advantage of mental time travel is the increased flexibility in acting in the present to secure future needs. First, therefore, one has to be capable of conceiving having different future needs, such as imagining being thirsty when currently quenched. One hypothesis, proposed by Bischof-Köhler (1985) and Bischof (1985), is that it is in fact an inability of nonhuman animals to flexibly entertain future need or drive states that limits their capacity for mental time travel. That is, nonhuman animals are unable to differentiate future states from present ones (Bischof-Köhler 1985; Bischof 1985; Suddendorf \& Corballis 1997). Superficially, at least, this hypothesis cannot be correct, since many species act to secure future needs, as illustrated by 
the caching of food. As noted, though, such behaviors may typically be largely instinctive, and experience may only allow changes within specific domain parameters. The Bischof-Köhler hypothesis may still apply to more individual, flexible situations involving non-instinctive behaviors. One study suggests that children anticipate future needs by around age 4 (Suddendorf \& Busby 2005), and the challenge is now to document its development in more detail and to test nonhuman species.

Nonverbal tests of the Bischof-Köhler hypothesis have been proposed (Suddendorf 1994; Suddendorf \& Busby 2005; Tulving 2005), but have yet to be carried out. They involve controlling a current need state, such as ensuring that the subject is no longer thirsty, and then providing an opportunity to secure a future need, such as obtaining a drink for a future situation that will induce thirst. There are various caveats to consider in order to rule out alternative explanations based on instinct or associative learning (Suddendorf \& Busby 2005). For example, instinctual explanations can be ruled out if the experimental scenario does not involve species-typical behavior and if similar problems can be solved in a variety of domains. If the same animal were to pass variations of such tests across different needs (e.g., hunger, thirst, temperature) and contexts (locations, time-intervals), the Bischof-Köhler hypothesis would become increasingly untenable.

Mulcahy and Call (2006) recently came closest to implementing such a test. They trained bonobos and orangutans to obtain grapes from an apparatus using a tool. Access to the apparatus was then blocked and the animals were presented with a selection of two suitable and six unsuitable tools which they could take into a waiting room from where the apparatus was still visible. An hour later, they were allowed back into the testing room and given access to the apparatus. In 7 out of 16 trials, on average, the apes carried a suitable tool into the waiting room and returned with it to obtain grapes an hour later. There were strong individual differences in performance, with one orangutan achieving 15 out of 16 correct. This orangutan and the best performing bonobo were then tested again, but with an overnight delay between tool selection and return. They still returned with a suitable tool in more cases than expected by chance. A third experiment showed that the apes could pass the task even when they could not see the apparatus during tool selection. The final control study investigated whether the animals merely associated the tool with the reward. Subjects again received a grape reward if they returned with the right tool, but were not actually given an opportunity to use the tool. Performance in this condition was poorer, suggesting that they did plan ahead in the other studies (Mulcahy \& Call 2006).

Nevertheless, there are some concerns about this conclusion (Suddendorf 2006). The same tools were appropriate over trials, so apes could have just learned to always return with these same tools. This highlights the importance of the final control condition designed to rule out explanations based on associations. However, this control condition was not given to the successful animals of Experiments 1 to 3 , but to a new group of four animals. Two of these never brought the tool back and hence could never have experienced the reward that may have facilitated performance in the previous studies. Thus, their data do not inform us about the power of the reward. The other two performed identically to two animals in Experiment 1. Thus, contrary to what the authors claimed, association cannot be ruled out (Suddendorf 2006).

But even if subsequent studies confirm that great apes can select and keep a different tool for a specific future use, it does not show anticipation of future needs as proposed by the Bischof-Köhler hypothesis. The "future need" potentially anticipated in these studies refers to the "need" for a tool to satisfy a current desire for the treat, not anticipation of a different internal drive or need state (e.g., such as a future desire that is different from present). The studies did not control or manipulate the drive or need state of the subjects, and it is not unreasonable to assume that they all had a desire for grape rewards throughout testing (Suddendorf 2006). Animals that are not capable of conceiving of future drive and need states would have little reason to concern themselves with a remote future, as all they would care about is satisfaction of current needs. More research is required to determine the extent of animal foresight, but at present the limit proposed by the Bischof-Köhler hypothesis has not been falsified.

We are not making these killjoy arguments because of any preconceived notion about the way the world should be. We would be delighted if it could be established that other species have mental time travel. It would be a serious challenge to many humans' anthropocentric worldview and would have profound moral implications (Suddendorf \& Busby 2003a). Those species that can be shown to ponder what has happened, and to speculate about what might happen in the future, would require fundamentally different welfare considerations. For example, they would suffer not just from current pain, but also from revisiting past and anticipating future pain (Lea 2001). At present, there is no need to abandon the null hypothesis, but neither are we in a position to firmly conclude that other animals do not possess this capacity. We admit that a substantial body of comparative data is required before it can be concluded that any trait is uniquely human (Hauser et al. 2002).

Given the selective importance of future-oriented behavior, species are likely to have evolved a wealth of different mechanisms. The growing research interest in the question of animal mental time travel may help identify and describe these mechanisms. Our closest surviving relatives, the great apes, may be expected to have precursors of the human ability (e.g., foresight limited to serving current needs). Mulcahy et al. (2005), for example, have shown that gorillas and orangutans can select an appropriate tool in one location to solve a problem at another location that was not simultaneously visible. There is a range of evidence that great apes can imagine alternative states of the world (see sect. 4.1) and may hence offer a link between animal and human minds (for a review, see Suddendorf \& Whiten 2001). It is possible, then, that antecedents of mental time travel had evolved in the common great ape ancestor. It has been argued that living in fissionfusion societies, as modern chimpanzees do, may have selected for an increased capacity to monitor across time and space the comings and goings of other group members (Barrett et al. 2003). Alternatively, ecological pressures on large arboreal apes, like modern orangutans, feeding on spatially and temporally variable food sources 
(e.g., fruit) might have led to selection for increased tracking of these patterns and planning of travel routes in our common ancestor. But it remains to be established what exactly the nature and extent of modern apes' foresight (and that of other animals) is. We maintain that the data so far continue to suggest that mental time travel is unique to humans. Next we make the case that future research should focus not only on purported instances of mental time travel, but also on subsidiary mechanisms that are typical of human mental time travel and that may be necessary to sustain it.

\section{Components of human mental time travel}

One reason to question whether mental time travel is possible in nonhuman animals is that flexible anticipation of particular events is no easy feat and may involve a suite of cognitive abilities that may not be available to animals or to young children (Suddendorf \& Busby 2003b; Suddendorf \& Corballis 1997). Failures in any one of these may lead to disappointed expectations (a source of much human misery and, we may add, many a punch line) or to no anticipation at all. To evolve a flexible anticipation system, many cognitive components may need to be in place to achieve a level of accuracy that provides a selective advantage sufficient to compensate for the enormous expense of cognitive resources. An appropriate metaphor might be a theater production, which also requires many subsidiary activities apart from the production itself. In the words of Shakespeare, "All the world's a stage," and this, we suggest, may include the mental world.

In likening mental time travel to a theater production, we are not making any claims about a Cartesian theater of the mind, as discussed by Dennett (e.g., 1995), nor about simulation or any other mechanisms by which this may be instantiated in the brain. Mental simulation of perception and action is of course one way of thinking about conscious thought in general (Hesslow 2002) and mental time travel in particular (Suddendorf 1994), but the point of the analogy here is simply to draw attention to the range of mental capacities that might be considered necessary to engage successfully in mental time travel. We discuss the main theater components and their cognitive analogs in the following sections.

\subsection{The stage}

To entertain a future event one needs imagination - some kind of representational space in our mind for the imaginary performance. In cognitive psychology the concept of working memory is usually conceived of as such a space (or workbench) where information is temporally combined and manipulated (Baddeley 1992). Human working memory appears to comprise, in addition to a central executive, two distinct subsystems: a phonological loop and a visuospatial sketchpad. Although non-lingual species may not have anything like the inner voice and inner ear (i.e., the phonological loop), they may very well have an image-based system (i.e., the visuospatial sketchpad).

To represent the future, the stage has to allow combination not only of recently presented material, but also of information stored in long-term memory. It must allow offline processing. Dreaming is an example of offline processing, and mammals appear to have dreams. Offline replay during sleep may be involved in consolidation of declarative memories in the hippocampus (Kali \& Dayan 2004; Wilson \& McNaughton 1994). In fact, dreaming or day-dreaming may be regarded as a form of "scenario building" that social mammals may have evolved to allow practice/play without suffering consequences (Alexander 1989).

To make such offline processing, or mental play, directly relevant to situations, however, one also has to be able to collate it with perceptions of the present real world. So-called secondary representations are thought to allow just that (Perner 1991; Suddendorf 1999). In addition to a primary representation of current reality, a secondary representation of a goal constellation, for example, allows an individual to test potential moves by mental rather than physical trial and error. This capacity is also evident in pretend play (where one entertains an imaginary world while maintaining awareness of the real), in Piagetian invisible displacement tests (where one needs to reason about an invisible past trajectory of a target), and in various other basic cognitive tasks. During the second year of life, human children begin to display evidence for secondary representation in each of these contexts (Perner 1991; Suddendorf \& Whiten 2001). A review of the literature found that only our closest relatives, the great apes, have so far provided some evidence for secondary representation in all of these areas (Suddendorf \& Whiten 2001; 2003; Whiten \& Suddendorf, in press) and may therefore have the mental stage to imagine past or future events. Though convergent evolution may have produced similar skills in other species (e.g., corvids; see Emery \& Clayton 2004), our own capacity for secondary representation appears to have evolved in the common great ape ancestor some 14 million years ago (Suddendorf \& Whiten 2001).

\subsection{The playwright}

To generate content, imaginary events need a script or narrative. This requires access to a declarative database - that is, stored information that is not stimulus bound, but can be made available top-down. As noted earlier, some nonhuman species appear to have declarative memory that could serve this function. To generate new content, however, one further needs to be able to combine and recombine existing elements. Like language, imaginary narratives may operate according to the principle of "discrete infinity" (Hauser et al. 2002), involving the recursive application of rules to create an unlimited set of potential future scenarios (Suddendorf \& Corballis 1997).

Recursion is a computational procedure that calls itself, or calls an equivalent kind of procedure - as exemplified in sentences with embedded clauses, such as "The malt that the rat ate lay in the house that Jack built." Recursion lies at the heart of grammar, and enables us to create, from a finite set of elements, a potential infinity of sentences that convey a potential infinity of meanings. Recursion may also be said to underlie other aspects of human thought, including perhaps music, manufacture, navigation, social relationships, and numbering systems (Corballis 1991; Hauser et al. 2002). Theory of mind, which is the 
understanding that others have beliefs, desires, or intentions, for example, can involve several levels of recursion, as in such propositions as I think that you think I think you're stupid, which involves double recursion. Counting, as distinct from subitization or number estimation, involves principles that can be used recursively, enabling us to enumerate indefinitely. We humans, at least, use the concept of time in recursive ways, as in our understanding of such sentences like "Next Thursday he will have arrived in Mexico." The future perfect is but one of some 30 different tenses in English, and reflects the close relation between language and mental time travel. Children begin to show a recursive, generative capacity in the early preschool years.

There is as yet little, if any, evidence for recursion in any nonhuman species (Corballis 2003). Many birds sing songs of considerable complexity, and individual birds may have repertoires of many songs, involving different sequences of notes, but individual songs tend to be fixed and are repeated with remarkable consistency (e.g., Kroodsma \& Momose 1991). There has been at least one claim that birds can generate songs using grammatical principles: Hailman and Ficken (1986) reported that the songs of chickadees follow rules governing the selection and sequencing of notes, but those rules appear to conform to a simple finite-state grammar rather than a contextfree grammar like that underlying human language (Corballis 1991, p. 140). There is no evidence of recursion.

A recent claim that starlings can learn to recognize recursive patterns of sounds (Gentner et al. 2006) is, in our opinion, without foundation. The experimenters generated recursive sequences of up to eight sounds by embedding pairs of sounds, each consisting of a rattle and a warble, into another pair in recursive fashion. For example, if $A_{i}$ denotes a rattle and $B_{i}$ a warble, two levels of embedding would create the sequence $A_{3}\left(A_{2}\left(A_{1} B_{1}\right)\right.$ $\left.B_{2}\right) B_{3}$. Exact sequences were seldom repeated, since the actual sounds were randomly selected from a population of eight rattles and eight warbles. The birds were able to discriminate sequences based on this recursive embedding from sequences not obeying the rule. This need not mean, though, that they understood recursion, since each sequence could be regarded as simply comprising a sequence of rattles followed by a sequence of warbles (e.g., $A_{3} A_{2} A_{1}$ followed by $B_{1} B_{2} B_{3}$ ). That is, the birds may simply have established the number of sequential rattles and the number of sequential warbles, and responded positively if the two were equal. Number perception for auditory sequences in birds may actually surpass that in humans (Thompson 1969), so a strategy of "estimateand-match" may well have been within the capacity of the starlings. An earlier study showed that tamarins were unable to perform an analogous discrimination task (Fitch \& Hauser 2004), but even if they had been able to make the discrimination, it need not have implied that they recognized recursion itself (see Corballis [in press] for more detailed discussion).

\subsection{The actors}

Events typically involve characters, and mental time travel might well have evolved in order to predict (and aid or thwart) the behaviors of others (discussed further on in this section). To represent self and others realistically requires declarative knowledge of individuals and some folk psychology (i.e., theory of mind) to predict how they act (e.g., knowing that they typically act on the basis of their beliefs to fulfill their desires). With such knowledge, one can engage in more sophisticated social competition and cooperation, and one of the most effective ways to do so is to act like an actor: "to see ourselves as others see us, so as to cause them to see us as we would like them to rather than as they would like to" (Alexander 1989, p. 491). Self-awareness is difficult to define and measure. Alexander (1989) argues that one important aspect of self-awareness springs directly from an ability to consider one's self in various alternative versions of future events. This is the notion of "free will," implying that one can deliberately choose to pursue one of several entertained paths.

It is established that great apes, like 2-year-old children and unlike many other animals tested, can at least recognize themselves in mirrors (e.g., Bard et al. 2006; Gallup 1970; Patterson \& Cohen 1994; Suarez \& Gallup 1981). They have an expectation of what they look like from the outside, and this expectation is rapidly updatable (Nielsen et al. 2006). Together with evidence for some understanding of what others can see (Call et al. 1998; Hare et al. 2000 ) or did see (Hare et al. 2001), this strongly suggests that they can "see themselves as others see them" at least in the literal sense. But it is not so clear whether they care about manipulating how others see them (even in this literal sense, only humans regularly adorn themselves with jewellery and clothes).

Nevertheless, there is a sizable body of evidence to suggest that primates do engage in various forms of tactical deception in social interactions (Whiten \& Byrne 1988). Primate social knowledge is impressive. Many primates recognize other individuals, as well as their social rank and relations (Seyfarth et al. 2005). Great apes and 2-year-old children also appear to have some limited folk psychology, but the precise nature of their capacities remains to be established (e.g., Suddendorf \& Whiten 2003; Tomasello et al. 2003). Currently, the available data suggest that they do not have a representational theory of mind which includes the capacity to metarepresent that others may hold representations contradicting their own (Call 2001; Whiten \& Suddendorf 2001). It is only between ages 3 and 4 that humans pass tests (e.g., false-belief tasks) that demonstrate such understanding (e.g., Perner 1991; Wellman et al. 2001). We have argued that it may require this level of folk psychology to be able to identify with one's future self, understand that this future self may have mental states that differ from one's current states, and care about them (Suddendorf \& Corballis 1997). As Hazlitt (1805) observed: "The imagination ... must carry me out of myself into the feelings of others by one and the same process by which I am thrown forward as it were into my future being, and interested in it" (p. 1).

\subsection{The set}

The mental play also needs a physical context that operates according to real-world principles. This requires some "folk physics." Although one prominent researcher recently concluded that not even apes can mentally represent unobservable causal factors such as gravity and 
force (Povinelli 2000), many other researchers are not convinced that their folk physics is quite so limited (Allen 2002; Hauser 2001; Whiten 2001). Chimpanzees, for example, have passed double invisible displacement (object-permanence) tasks that require reasoning about the movement of objects that are no longer perceptible (Collier-Baker \& Suddendorf 2006).

Especially important to mental time travel is some appreciation of the time dimension itself. Some fundamental timing processes (e.g., circadian rhythms) that link behavior to regular environmental changes are clearly widespread in the animal kingdom and are fairly well understood (e.g., Albrecht \& Eichele 2003). Other, more cognitive processes are less well understood. There appear to be various different timing mechanisms in the human brain that track key information about temporal distance, order, and location within time patterns and humans appear to draw on different combinations of those (Friedman 2005).

Proposals for time measurement mechanisms range from discrete accumulating neural pacemakers (e.g., in scalar expectancy theory) to models involving continuous decay strength of memory traces. As noted earlier, fading of memory may itself provide temporal information that could account for the when component of www memory in scrub jays. In humans, cognitively controlled, rather than automatic, tracking of the passage of time typically activates the right hemispheric dorsolateral prefrontal cortex, an area also implicated in working memory (Lewis \& Miall 2006). Lewis and Miall argue that both working memory and judging temporal distance may depend on the same dopamine-modulated neuronal system. Children begin to accurately discriminate distances of past events from around age 4 onwards (Friedman \& Kemp 1998).

Mental time travel may itself make such timing based on memory-decay functions less reliable. Since mentally constructing and reconstructing episodes reactivates memory traces, it may strengthen these memory traces and make them appear to be "fresher" in memory than warranted by age alone. Other timing mechanisms may therefore have evolved for the construction of a more accurate chronology. Indeed, chronology appears not to be a basic property of human memory, but rather, depends on active repeated construction (Friedman 1993). Some information about the order in which events occurred (e.g., before and after) may, however, be stored in memory. As we saw earlier, even rats show evidence of tracking temporal order (Eichenbaum et al. 2005).

How internal clocks, order codes, or other processes give rise to adult human concepts of the time dimension remains unclear. Human cultures have developed different semantic representations of time patterns (e.g., hours, days, weeks, months, etc.) that enable people to locate the time of events. Actively reconstructing locations of events in such explicit time patterns is probably the most common human approach to timing (Friedman 1993). Location-based processes are evident in scale effects where judgment of time of day of a past event may be more accurate than judgment of the month in which the event occurred - distance-based mechanisms cannot explain such phenomena. Friedman (2005) recently reviewed the evidence and concluded that humans use both verbal and image-based processes to reason about the temporal location of events and that children use image codes to differentiate daily events by around age 5, and verbal lists, such as days of the week, by age 7. Children's relatively late acquisition of cultural time concepts suggests that they are not easy to acquire.

Once established, such time patterns offer the opportunity to place future events at particular points in time. Of course, neither order codes nor distance information in memory can explain the development of a temporally differentiated sense of the future. Children begin to show a rough temporal differentiation of future events by around age 5 (for a recent review, see Friedman 2005). Nonhuman animals seem severely limited in learning temporal information, and performance appears to be based on non-declarative mechanisms, rather than any such explicit concepts of time (Roberts 2002). Nonetheless, the work on scrub jays reviewed earlier suggests temporaltracking mechanisms more sophisticated than previously thought to exist in any nonhuman species.

\subsection{The director}

Mental time travel is of course not to be mistaken for clairvoyance. The future contains many possibilities, and hence mental time travel involves entertaining different versions of scenarios and evaluating their likelihood and desirability. Like a director trying out alternative ways of presenting a scene, effective mental time travel requires rehearsals and evaluations. This entails some level of dissociation and metacognition (i.e., thinking about thinking), which first emerges in children around age 4 (Perner 1991; Suddendorf 1999; Suddendorf \& Corballis 1997). There is at best limited evidence for very basic metacognitive capacities in nonhuman animals (Hampton 2001; Shettleworth \& Sutton 2006).

In studies involving extensive training on a simple task, such as determining which of two stimuli is the larger, some monkeys given the option of not taking a trial eventually learn to avoid trials they are likely to fail (Smith et al. 2003; Son \& Kornell 2005). Such uncertainty monitoring arguably implies metacognition, in that the monkeys may know that they do not know, and may even imply selfreflective consciousness. However, Son and Kornell concede that their results need not mean that monkeys are aware of their judgments. It remains unclear to us how any such performance is "meta" over and above other undisputable and common confidence judgments that animals may hold about their own ability. For example, judging how far it can jump is surely essential for any monkey traversing the canopy. The nature of what is supposed to be (meta-) represented may play a role in comparative cognition. Great apes have shown some signs of competence in regard to basic mental states. Call and colleagues (Call 2004; Call \& Carpenter 2001), for example, recently provided some evidence that great apes know something about what they have or have not seen, as reflected in whether they do or do not seek further information before acting. But Call (2005) notes that it is not known whether any nonhuman species attribute more complex states such as knowledge or beliefs to themselves.

A key role of a director is to orchestrate rehearsals. Humans rehearse significant future events and practice their own performance not only in their minds, but also in action. In fact, this trait would seem essential to any 
account of the enormous diversity of human expertise. Humans choose to learn and practice things they want to get better at. A function of play in many species may be that it prepares the young for the future (e.g., fighting skills). However, unlike species-typical play, humans choose what to practice and often do so in anticipation of specific future events (e.g., rehearsal for a theater performance). We know of no evidence that nonhuman animals deliberately practice for specific anticipated events (Suddendorf \& Busby 2003b).

\subsection{The executive producer}

Enacting a planned event requires voluntary control, including executive functions such as the ability to inhibit other stimulus-driven responses in favor of one that suits the anticipated events best. Impaired mental time travel capacity following frontal-lobe damage has been associated with impairment of such self-regulation (Levine 2004). Even inhibiting a simple response in order to increase one's total future reward is a difficult task for young children, as research on delay of gratification amply illustrates (Mischel \& Mischel 1983; Moore et al. 1998). Adult humans, on the other hand, regularly forego instant rewards because of a multitude of anticipated long-term gains (e.g., weight loss, longevity, afterlife).

To override current drives in favor of acting to secure some anticipated future outcome, it would help to overestimate the positive or negative effects of future reward or punishment. In fact, humans show a systematic bias towards overestimating the intensity of future affective responses to future events (e.g., the dreaded interview with the bank manager often turns out to be more benign than expected). The nature of this biased "affective forecasting" is currently a topic of investigation in social psychology (e.g., Gilbert 2006; Wilson \& Gilbert 2005). Accurately anticipating one's own future mental states is no easy feat and is often influenced by one's current states - which is why one buys more treats when shopping while hungry (Gilbert et al. 2002). Even children show this effect (Atance \& Meltzoff 2006).

Plans further require prospective memory - remembering to perform a future action at the appropriate time. Purported evidence for prospective memory in nonhuman animals has been disputed (Thorpe et al. 2004). Executive control may be required not only to implement strategic action plans, but also to manage the motivational and goal system itself (Conway et al. 2004; Suddendorf \& Busby 2005). For mental time travel to become the flexible and effective future-oriented strategy that it is, cognition had to truly take the driver's seat of behavior (Suddendorf 1999). Modern humans often juggle a multitude of different goals and must decide when to do what to achieve which aims. The development of these sophisticated, resource-intensive, and error-prone mechanisms continues well into adulthood, and we know of no evidence for a comparable capacity in nonhuman animals.

\subsection{The broadcaster}

Finally, temporally displaced events are not only entertained in private but are often communicated. A play is not just a metaphor for mental time travel, it is often also a public expression of it. More generally, humans use language to exchange and complement their mental travels into the past and their ideas about future events, as well as to cooperatively coordinate plans and strategies (Suddendorf \& Corballis 1997). There is as yet no evidence that nonhuman animals communicate such mental travels. Such communication does not require language, as pantomime and dance can demonstrate. Chimpanzees appear to have some capacity to imitate (Whiten 1998) and to recognize when someone else is copying them (Nielsen et al. 2005). Animals with such abilities might in principle also be able to re-enact past episodes, using mime, if they wanted to communicate those to others.

Although language is not necessary for mental time travel, it provides the clearest evidence of it. Indeed, it may not be too far-fetched to suppose that mental time travel was a prerequisite to the evolution of language itself, since, as we saw earlier, language is exquisitely equipped to express events that are distant in both space and time from the present. If we are correct in supposing that mental time travel is uniquely human, then this may explain why language itself is unique to our species, at least in the strong sense defined by Hauser et al. (2002). The adaptive utility of the capacity to transcend the present in an open-ended, flexible manner may have driven these same properties in language (for a discussion, see Corballis \& Suddendorf, in press).

\subsection{Components of successful mental time travel}

In summary, the theater metaphor implies that mental time travel requires a constellation of skills and is not simply an isolated capacity. Adult humans may fail to act now for a particular future event because of a deficiency in any one of these components (e.g., one may fail to predict one's own future mental states, or one may fail to override more immediate impulses). Young children may also fail to do so because one or the other of these components is not yet fully developed. Further study of how these requisite capacities emerge in human development may hold clues to the relationship between them.

Some of the foundations, such as imagination, selfrecognition, and semantic memory, begin to emerge early in childhood; whereas other capacities, such as recursive thought and representational theory of mind, develop first in children between ages 3 and 4 . It is around this latter time that unequivocal evidence for episodic memory emerges, childhood amnesia ceases, and implicated prefrontal regions mature (e.g., Levine 2004). Between ages 3 and 5 , children start to reason about future and past states (Atance \& Meltzoff 2005; Gopnik \& Slaughter 1991; Suddendorf \& Busby 2005), make plans (Atance \& O’Neill 2005; Hudson et al. 1995), delay gratification (Mischel \& Mischel 1983; Thompson et al. 1997), accurately report events of yesterday and tomorrow (Busby \& Suddendorf 2005; Harner 1975), and temporally differentiate events in the past (Friedman \& Kemp 1998) and in the future (Friedman 2000)

Nonhuman animals may also be limited because of deficiencies in any of these components. Although there is evidence in some species (especially from our closest relatives) for basic components, such as imagination and self-recognition, support for most others is very slim. Should a component be present only weakly, if at all, in 
other species, mental time travel would be severely limited, at best. For example, without a representational theory of mind, foresight might be restricted to serving satisfaction of current needs, as the Bischof-Köhler hypothesis proposes. Our suggestion, then, is that when studying the possibility of mental time travel in other species, the research agenda should include work on these components to human mental time travel. Determining to what extent specific components are necessary and sufficient for what aspects of foresight to emerge will be an important task.

\section{Some qualifications}

Our theater metaphor and our reference to constructing particular events, as well as the notion of www memory, have connotations that may be misleading. We are not suggesting that humans typically re- or pre-construct full-fledged episodes that are played out like a video recording. As pointed out earlier, episodes are actively constructed and are prone to error. Also, mental time travel often involves only the smallest snippets of events. In fact, the snippets do not have to come from the same place and time; snippets from very different times can be strung together. That is, humans can relate events from different times and identify patterns that help us make sense of the present (e.g., "he did X, and then two days later Y, only to now do Z") and to predict the future (e.g., "I am going to do A tomorrow, which will deal with his action Y, so I can achieve B next week").

Rats, on the other hand, even after thousands of trials fail to learn the link between a stimulus and a reward if they are a few seconds apart, and there is no secondary reinforcer (Grice 1946, cited in Roberts 2002). Relating events across time may be one of the key advantages provided by mental time travel into the past, as it offers humans additional information for prediction of events to follow. In other species, solutions to the need to bridge temporally distant events are typically highly specific and lack the flexibility of mental time travel. Whereas most learning links events that occur within a few seconds of each other, rats famously can link taste with nausea even when the illness develops hours after consumption (Etscorn \& Stephens 1973; Garcia et al. 1966). This linkage, however, appears to be highly specific, and is limited to the linking of sickness to taste, and not to auditory or visual stimuli (Garcia \& Koelling 1966). This temporally extended learning mechanism thus appears to have evolved in response to a recurring problem with strong selective force (those who eat too much of the wrong food die). Humans appear to be less constrained and can (though may not) link virtually anything to anything else across time.

Although we have highlighted the similarities between mental time travel past and future, there are of course also some notable differences. Obviously, the past has in fact happened, whereas the future harbors a lot more uncertainty. For example, the weather may change, or the car may break down. Another potential difference lies in the temporal distribution of the number of events people recall and anticipate. Although there are similarities in the distributions (Spreng \& Levine 2006), the past features the so-called reminiscence bump - by the age of around 70, for example, people tend to recall more events from their twenties than events from their forties and fifties (Rubin \& Schulkind 1997). Our view of mental time travel may provide an answer as to why that may be so. If the key function of mental time travel into the past is indeed to inform and allow the anticipation of the future, then it is in the formative years that people have to retain as much event detail as possible in order to abstract facts and make specific predictions. Accumulating a vast pool of episodes of varied experiences provides one with an arsenal of information for future prediction.

As one matures, one also accumulates more semantic facts, and fewer things are surprising. Predictions can increasingly be made by abstract rules (i.e., using semantic prospection) rather than through imagination of individual future events. Retaining details of individual events becomes less important the more over-arching principles have been deduced. Good prediction depends on the right balance of semantic and episodic information, so that one does not get bogged down in too much episodic clutter (e.g., Anderson \& Schooler 1991). Episodic memory may decline as a consequence of the increase in the extraction of facts and rules, or what some would call crystallized intelligence. Thus, although we have argued that semantic memory and prospection are phylogenetically older than episodic memory and prospection, mental time travel may vastly increase the nature and capacity of semantic future-oriented cognition. Just as semantic and episodic memory interact (Tulving 2005), so may semantic and episodic prospection.

\section{Evolutionary considerations}

Because behavior that increases future survival has a selective advantage, mechanisms for future-directed behavior are ubiquitous. The now-standard taxonomy of human memory, we suggest, can be extended to incorporate variations in the degree of flexibility each system provides for the future. Mental time travel offers the ultimate level of flexibility, allowing voluntary anticipation of any particular event. This foresight requires several sophisticated cognitive abilities, is resource-intensive and error-prone, and introduces new kinds of mental stress, not least of which is the knowledge of inevitable death.

This is a high price to pay for a system for anticipating the future, which of course can never be known with perfect precision, especially considering the fact that much can be achieved with simpler prospection systems (instincts, procedural and semantic prospection). But mental time travel offers an additional degree of flexibility that, even if the adaptive advantage was in the first instance small, was sufficient to ensure selection and fixation in the population. Haldane (1927) computed that a variant resulting in a mere $1 \%$ in fitness would increase in population frequency from $0.1 \%$ to $99.9 \%$ in just over 4,000 generations, a time span that fits easily into hominin evolution, or even into the evolution of our own genus, Homo. Even if the initial adaptive advantage may have been small, it is clear that today humans base much of their actions on anticipation of future events, from preparing this weekend's escape to building wealth. 
The immense flexibility this foresight provides may have allowed us to successfully adapt to and colonize most habitats on the planet (Suddendorf \& Busby 2005). Our futuredirected behavior can even go beyond merely individual needs and may carry a concern for the planet itself, along with its inhabitants. That concern may also extend beyond the life span of the individual. As perhaps the only species with such foresight, humans alone may be driven to consciously guide the planet into the future (Dawkins 2000) and thus be burdened with the responsibility of getting it right. We can identify future threats to our world, be they of our own doing (e.g., the consequences of human-induced global warming) or of external origin (e.g., an asteroid on collision course with Earth). Humans can forecast the outcomes and choose to act now to secure future needs. Cultures have evolved complex moral systems that judge actions as right or wrong partly based on what the actor could or could not have reasonably foreseen to be the future consequences of the act. Law, education, religion, and many other fundamental aspects of human culture are deeply dependant on our shared ability to reconstruct past and imagine future events.

So why might humans, but not other animals, have the capacity for mental time travel? Are there plausible accounts as to how it may have evolved since the split from the last common ancestor of Homo and Pan some 5 or 6 million years ago? The possibility of a gap between the human and animal mind need not contradict Darwinian principles of natural selection. With the global shift to cooler climate after 2.5 million years ago, much of southern and eastern Africa probably became more open and sparsely wooded, exposing the hominins to greater danger from predators and forcing them into a "cognitive niche" (Tooby \& DeVore 1987), with heavy reliance on social cooperation and communication for survival. With the emergence of the genus Homo, brain size increased rapidly from around 2 million years ago (Wood \& Collard 1999), possibly reflecting selection for such interrelated attributes as theory of mind, language, and, we propose, mental time travel.

The earliest hard evidence, quite literally, for foresight comprises stone tools that appear to have been transported for repeated use. Reconstruction of knapping routines (using refit data) suggests that at least by the Middle Pleistocene hominins produced stone tools in one site to use them later at another (Hallos 2005). Bipedal hominins in the deforested savannah may have relied increasingly on throwing stones at predators (e.g., Calvin 1982), as well as using them to bring down prey and cut up carcasses. Great apes do hurl objects, but with little precision. Carrying rocks for use as missiles at some future point may have been vital, and a capacity to plan for this might have been strongly selected for. By 400,000 years ago some hominins had clearly developed aimed throwing, as revealed by wooden spears uncovered in Germany together with butchered horses (Thieme 1997). One possibility, then, is that extensive foresight evolved first in the context of throwing, as hominins faced different predators and prey in the savannah than they did in the trees. Osvath and Gardenfors (2005), for example, marshal evidence for such a scenario leading from the Oldowan tool culture to the evolution of mental time travel.
Similar arguments could be made for other ways in which hominins may have first adapted their environment to suit their future needs. The fact that stone tools provide us with an archaeological record that other behaviors do not may skew our evaluation of their significance. Consider, for example, the use of fire, which is also evident by 400,000 years ago (e.g., Boaz et al. 2004) and may well have been used much earlier. Although it is difficult to identify archaeological evidence for mastery of fire, one needs little imagination to envisage the huge selective advantage that it might have bestowed, in defense, attack, cooking, provision of warmth, night-time vision, and so on. Planning capacities could have been selected for as the incidental use of fire gave way to maintenance of fire and, finally, to the making of fire for more controlled and deliberate purposes. Perhaps the ancient Greeks were right: They believed that Prometheus stole fire from heaven to give humans the powers of the gods that set them apart from animals - Prometheus means foresight.

Yet, these victories in gaining control of the natural world may only have been side effects of a quite different evolutionary arms race. Technological advances may have been secondary to abilities that evolved primarily in the social arena. Modern humans certainly use their planning capacities extensively to cooperate with, deceive, and manipulate conspecifics. As noted earlier, much of what humans recall and foresee has to do with "who did what to whom, when, where, and why" (Pinker 2003, p. 27). It has been suggested that social rather than ecological pressures drove the evolution of intelligence, not only in humans, but in primates more generally (Humphrey 1976). This hypothesis has attracted some strong support in recent years (e.g., Dunbar 2003; Seyfarth et al. 2005; Whiten \& Byrne 1988).

One problem for such an account is to explain why the cognitive arms race seems to have persisted longer in humans than in other primates, resulting in apparently unique cognitive skills, including, perhaps, mental time travel. A potential explanation, suggested by Alexander and colleagues (Alexander 1989; Flinn et al. 2005), is that once early hominins obtained a certain level of "ecological dominance," perhaps partly through technological advances as discussed earlier, they were faced with increased competition from their own species - "humans uniquely became their own principal hostile force of nature" (Alexander 1989). This may have resulted in a runaway social competition between (and within) groups towards greater intelligence, and enhanced abilities for both cooperation and deception. These may have included the ability to entertain alternative future scenarios (mental time travel), to read others' minds (theory of mind), and to communicate (language).

This "coalitionary arms race" (Flinn et al. 2005) also offers an answer to one of the greatest mysteries of human evolution: Why have all the other hominin species become extinct? One only needs to extend the proposal from "intra-species" competition to "intra-genus" competition to see a solution. Our ancestors may have competed with an array of other bipedal species that once graced this planet, and perhaps simply outsmarted them, contributing to their demise (Suddendorf 2004). One of the final steps that may have given us a decisive edge over one of the last nonhuman hominins, the Neanderthals, may have been 
autonomous speech (Corballis 2004). This suggestion that humans played a role in displacing other hominins is in line with recorded human history in that human groups perpetually compete with each other and often violently displace other groups, as well as with various other human characteristics, such as our unique obsessions with inter-group competitive play (e.g., sports). This intra-genus competition may have ultimately been won by human groups because of continued advances in foresight, language, culture, and coordinated aggression, leaving us as the sole survivors of an extraordinary evolutionary arms race. We may be the only species capable of mental time travel because the others competing in our niche have become (or have been made) extinct.

Like the "just-so stories" about throwing or fire, an account emphasizing intra-species or intra-genus competition may exaggerate the importance of a single factor. Claiming that the hostile forces of nature turned into "relative trivialities" (Alexander 1989) probably underestimates the selective pressure of catastrophic environmental changes such as ice ages, volcanic eruptions, and meteorite impacts on life in general. Such events may well have produced bottlenecks in evolutionary history where social competition was of far less import than ecological factors and cooperation. Rather than endorsing one or the other scenario, we present them here merely to make the point that plausible accounts exist that can explain why humans may have capacities, such as mental time travel, that other species do not have. Darwinian continuity need not demand greater mental powers in nonhuman animals than is currently evident.

\section{ACKNOWLEDGMENTS}

Thomas Suddendorf was supported by Australian Research Council Discovery grants (DP0557424 and DP0770113). We are thankful to the eight anonymous reviewers for providing useful comments on an earlier version of this manuscript.

\section{Open Peer Commentary}

\section{Foresight has to pay off in the present moment $^{1}$}

DOI: $10.1017 /$ S0140525X07001987

\section{George Ainslie \\ Veterans Affairs Medical Center, Coatesville, PA 19320. \\ George.Ainslie@va.gov picoeconomics.com}

\begin{abstract}
Foresight requires not only scenarios constructed from memories, but also adequate incentive to let these scenarios compete with current rewards. This incentive probably comes from the efficacy of the scenarios in occasioning present emotions, which depends not on their accuracy per se but on their uniqueness as compared with other possible occasions for emotion.
\end{abstract}

The theater analogy provided by Suddendorf \& Corballis (S\&C) in the target article is an apt one. Whereas a species is selected for adaptation to its environment over generations, the behaviors of an individual are selected for their reward (or entertainment) value in the here-and-now. Any policies that extend over time must compete for acceptance in the present moment, and the process that represents them in the present moment could well be described as either dramatization or time travel. ${ }^{2}$ For nonhumans, or at least non-primates, the entertainment is of a concrete nature - mating, the hunt for food, the urge for rage - and the times traveled are on the order of seconds. As the authors note (sect. 1), longer policies have had to be genetically hardwired, in the form of urges to hoard or migrate, so as to demand no more time travel than that. The time travel process is still necessary for those species, however, and is visible in the behaviors that used to be called vicarious trial and error (Tolman 1939). Conversely, time travel is conspicuously absent in hungry animals with ablations of the ventral striatum, which will eat food that is next to them but not walk even a few inches to get it (Berridge \& Robinson 1998). Even a rat needs some form of imagination.

As scenario-writing extends further into the future, it encounters a design problem: how to interest the present agent in longdeferred prospects. The single-digit annual discount rates that are adequate to sell people secure financial investments clearly apply only to surplus wealth - that is, wealth beyond what is needed to sustain current hedonic tone. Four-year-old children, who can metarepresent others' beliefs (sect. 4.3) and tell distances to past events (sect. 4.4) still have difficulty waiting a few minutes to get two marshmallows instead of one (Mischel \& Mischel 1983). Even adults have little tolerance for the boredom of a bad lecture or getting stuck in traffic, times when our usual supply of entertainment is interrupted. Volunteer subjects will often not wait two minutes to quadruple their access to a video game (Millar \& Navarick 1984) and are similarly impatient to get relief from unpleasant noise (Navarick 1982). Playwrights notoriously have to design not just a plot that develops over two hours or so, but smart dialogue that provides payoffs from minute to minute (cf. the "flip value" required of novelists). Figuratively and literally, the S\&C theater model of foresight is missing a key element - the audience, the present self that chooses the most rewarding time travel available. The author, producer, and director cannot impose their scenarios on the paying public, but must compete on the basis of entertainment value. This means that a future scenario must compete with current comfort, and at a substantial discount. At the future discount rates implied by people's patience for actual discomfort, the conventional exponential formula makes the value of an experience that is even a few days away infinitesimal. The relatively high tail of hyperbolic discount curves raises the value of distant events, but still not enough for events that will happen after days to compete with events that will happen after minutes (Ainslie 2006). Time travel has to bring into the present not only the picture of future events, but also a significant share of their likely emotional impact.

In effect, the discounted value of distant scenarios has to be amplified if they are to compete with scenarios that are at hand. The most likely mechanism is emotion. Strong feelings can be occasioned by stories, sometimes by mere symbols; and, as social constructionists have long pointed out, such "texts" are highly manipulable. However, the very flexibility of emotion creates the problem of separating useful amplification from noise. To say that actively constructed "episodes" are prone to error (sect. 5) is a great understatement. When evolution gives individuals arbitrary access to reward, it creates a design problem even in nonhuman animals: Dogs copulate with knees, for instance, and monkeys masturbate copiously, diverting sexual reward from its adaptive function. In imaginative humans the potential scope of the self-reward problem is illustrated by the powers of the fantasy-prone, the $1 \%$ or $2 \%$ of the population who are reported to enjoy imagining food as much as eating it, and to reach orgasm without physical stimulation (Rhue \& Lynn 1987). Lacking the usual habituation of their fantasies, these people report great difficulty in pursuing tasks without distraction. They demonstrate a factor that may have limited the evolution of intelligence: the concomitant ability to 
suborn reward from its adaptive purposes. ${ }^{3}$ For brain power not to mainly produce more efficient autists, something has to make emotions at least roughly model the distant scenarios that they are to amplify.

I have argued elsewhere that the crucial factor is the uniqueness of the occasions presented by these scenarios (Ainslie 2001, pp. 175-86). Trying to maximize prospective reward in distant scenarios becomes a game for present entertainment, which is how winning immediate coupons for merchandise to be delivered later can excite the brain as if it were a visceral reward (see, e.g., McClure et al. 2004). Scenarios that have unique criteria for winning or losing - determination by someone else, reality testing that stands up to varied approaches, a single long-held belief, and so on - become selected because they occasion reward more effectively, that is, because they make the present game better. It does not matter whether the scenario is realistic per se - someone else's novel has more power than your own daydream - but the personal rules that constrain predictions to be realistic are a major source of unique scenarios. Again, it does not matter if the rules are wrong, as long as they yield unique results. A shared cultural belief about what heaven is like and who is apt to go there may generate time travel that is as competitive as science. However, sources of unique occasions other than objective predictions will obviously reduce a person's adaptiveness.

Two factors that seem to help focus the amplification process are the habituation of emotion and the preparedness to have vicarious experience. The random imaginings of fantasy-prone people habituate too little, as noted earlier. The lack of empathic readiness in autistic people not only reduces social effectiveness but may interfere with time travel to their own futures, as the authors imply. They make a suggestion for future time travel that was also made by Julian Simon (1995), and which they were able to find in Hazlitt (1805; see target article, sect. 4.3): that a person "identify with one's future self," that is, vicariously construct the experience of future selves as if of other people.

It is true that "mental time travel cannot be defined in terms of the veracity of the content" (sect. 2.1), but veracity trades off with evocativeness in the contest for audience. Just as canons of believability in theater scripts range from farce (the most evocative but least probable assumptions) through well-made (less improbable but unbelievably neat) through realistic (believable but smoothed out by conventions) to naturalistic (could be mistaken for overhearing real life), people's practices of constructing foresight could be said to fall into hedonic accounts, comparable to the budgetary "mental accounts" described by Shefrin and Thaler (1988). Mere wishful thinking can be stiffened by rules for withholding immediate gratification to make daydreaming a robust activity; the possibility of coming true, however remote, promotes a daydream into quasi-planning status - hence, arguably, the attraction of the long-shot fortunes offered by lotteries; highly believable scenarios can make up for low evocativeness by their uniqueness; and certainty commits you to the discipline of "fact," even when an observer would call it delusion. As the authors say, the emergence of mental time travel has been a crucial step in evolution, but the choice among hedonic accounts that it makes possible has introduced new motivational complexities that have only begun to be studied.

\section{NOTES}

1. The author of this commentary is employed by a government agency, and as such this commentary is considered a work of the U.S government and not subject to copyright within the United States.

2. Time travel may seem to be a melodramatic term for the construction of imagination from memories, but it does capture the need for an active, and hence motivated, step, as opposed to passive perception.

3. It could be argued that this factor is still operating, since the smartest people do not have the most surviving offspring - by deliberate choice (Retherford \& Sewell 1989).

\section{How developmental science contributes to theories of future thinking}

\author{
DOI: $10.1017 /$ S0140525X07001999
}

\section{Cristina M. Atance ${ }^{\mathrm{a}}$ and Andrew N. Meltzoff ${ }^{\mathrm{b}}$}

${ }^{a}$ School of Psychology, University of Ottawa, Ottawa, Ontario, K1 N 6N5,

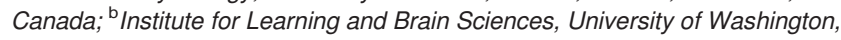
Seattle, WA 98195.

atance@uottawa.ca http://www.sciencessociales.uottawa.ca/ccll/ meltzoff@u.washington.edu http://ilabs.washington.edu/meltzoff/

Abstract: Acting in the present in anticipation of the future is argued to be a behavioral correlate of mental time travel (MTT). Yet, it is important to consider how other future-directed behaviors - including planning, delay of gratification, and acts of prospective memory - figure into a theory of MTT and future thinking more broadly. Developmental science can help in this formulation.

The authors of the target article have done an admirable job of describing the current understanding of the concept of mental time travel (MTT). Especially important is the creation of a taxonomy of future-oriented cognition because it will serve to further guide research in this area. However, the behavioral correlates of MTT (and specifically MTT into the future) require elaboration. Although the authors are clear about the various components of MTT, they are somewhat less clear about the various futureoriented behaviors that MTT undergirds. According to Suddendorf \& Corballis (S\&C), the sine qua non of MTT (into the future) is the ability to act in the present in anticipation of a need or state that is not currently experienced (e.g., imagining being thirsty when one is quenched). We are sympathetic to this view and agree that it is a powerful test of MTT. In fact, some of our recent research has explored this ability from a developmental perspective (Atance \& Meltzoff 2005; 2006). But, there are at least three reasons why neither we nor $\mathrm{S} \& \mathrm{C}$ should fixate on this one example alone.

Other markers of future-oriented behavior. First, as research with both adults (e.g., Gilbert et al. 2002) and children (e.g., Atance \& Meltzoff 2006; Suddendorf \& Busby 2005) demonstrates, this particular variety of MTT is extremely difficult - especially when one must anticipate a future state that directly conflicts with a current one as was the case in Atance and Meltzoff (2006). This might best be viewed as a high-end marker of MTT. But other markers also exist. For instance, it would also seem that the myriad of planning behaviors in which humans engage, along with the capacity to delay gratification and to remember to perform a future intention (i.e., prospective memory), all reflect MTT. Also important is whether there exist future-oriented skills crucial to human functioning that do not involve MTT. For example, the planning required to succeed on the Tower of Hanoi $(\mathrm{ToH})$ task is quite sophisticated, but it is unclear whether it requires the individual to actually mentally project the self into the future. On the other hand, planning what one will have for breakfast the next day seems much less taxing than planning moves on the ToH but may draw heavily on MTT. Developmental studies would provide useful evidence regarding which of these different tasks "cluster together." For instance, is the child who successfully reports what he plans to do tomorrow the same child who delays gratification now in order to obtain a larger reward in the future? Or, is the child who is able to report what she'll do tomorrow the same child who performs well on the ToH? Answers to such questions may aid in determining which behaviors reflect MTT, at which point in development they emerge, and provide hints about the neural systems required.

The importance of current desires for future success. Second, S\&C's criterion that a person acts in the present in anticipation of the future makes sense as applied to such visceral states as hunger and thirst, but it may not fully elucidate the cognitive 
complexities that underlie our strivings for more elusive states such as happiness, or our achievements of long-term goals such as job stability and the like. One of the most adaptive features of future thinking in humans is that we can hold in mind a desire or goal state and work tirelessly to achieve it. Part of the reason that this is possible is precisely because we experience the motivating desire or goal in the present. For example, some instances of prospective memory rely on the ability to translate a current desire into a plan for the future. That is, my (current) desire to take my medicine at 7 p.m. (future) ensures that at 7 a.m., I place my medicine beside my drinking glass. Similarly, when a child or adult is able to delay gratification, the desire for the larger (or end) reward is present. Arguably, it is when we lose sight, or stop experiencing, these drives and emotions that we cease to work towards them in a future-directed manner. Thus, it is important to consider not only how the anticipation of a future state can drive behavior in the present, but also how a current desire can ensure that an action, which brings the organism closer to a future goal, is carried out.

Partial knowledge and development. Third, there is the issue of partial knowledge. Even by age 5, children have not developed all the components that S\&C argue to be necessary for MTT. Are we to infer that prior to this age children are incapable of MTT? Although young children may not be able to consistently act in a manner that takes into account their future states, it seems overly conservative to argue that they are not capable of any form of MTT. True, some instances of MTT may require more advanced imaginative, executive, or theory of mind skills, but not all are required for a legitimate instance of MTT. For example, it seems plausible that a young child's inability to tell you what he's going to do tomorrow is not due to underdeveloped executive function skills but perhaps because of the division of labor in the family - mother does this planning. Careful developmental research would illuminate this and related issues. One could measure individual differences in executive function abilities and determine whether these predict individual children's performance on various MTT tasks.

Developmental science is crucial for constructing a more comprehensive theory of MTT. It will be important to develop tasks that not only test the child's ability to anticipate future states but also measure a number of other future-directed behaviors. In the early days of theory of mind research, a narrow fixation on children's understanding of false belief obscured the importance of many behaviors that form the foundation of a theory of mind, including joint attention, social referencing, and reasoning about desires and intentions. We should not make the same error again in the domain of future thinking.

\section{The continuum of "looking forward," and paradoxical requirements from memory}

\section{DOI: $10.1017 /$ S0140525X07002002}

\section{Moshe Bar \\ Martinos Center at Massachusetts General Hospital, Harvard Medical School, Charlestown, MA 02129. \\ bar@nmr.mgh.harvard.edu http://barlab.mgh.harvard.edu}

Abstract: The claim that nonhuman animals lack foresight is common and intuitive. I propose an alternative whereby foresight is a gradual continuum in that it is present in animals to the extent that it is needed. A second aspect of this commentary points out that the requirements that the memory that mediates foresight be both specific yet flexible seem contradictory.

Two primary issues are emphasized in this commentary. One is my alternative proposal that foresight should not be considered an all-or-none phenomenon, as suggested, but rather as a continuum. Second, I argue that the requirements from the memory that mediates foresight seem contradictory: It has to encode specific experiences, while at the same time it should be flexible enough to be applied to new situations. Keeping both issues in mind as this field advances will help fine-tune the welcome contribution of Suddendorf \& Corballis (S\&C).

The claim that foresight is unique to humans is common, and is based on reasonable intuition, but there is no evidence to support it unequivocally. In fact, as $\mathrm{S} \& \mathrm{C}$ review, evidence suggests that nonhuman animals might actually possess some limited foresight. For example, scrub jays show planning ability with their foodcaching behavior (Raby et al. 2007), fish can learn to anticipate feeding time and place (Reebs \& Lague 2000), rats have shown successful anticipatory behavior in response to complex feeding schedule (Babb \& Crystal 2006), and apes save tools for future use (Mulcahy \& Call 2006). Although these demonstrations do not necessarily reflect foresight as complex as ours, they nevertheless reflect future-related thoughts and actions. Do these mechanisms differ on a qualitative level from human foresight? The evidence does not support a definite positive or negative answer to this question. Nevertheless, given how much more sophisticated and intelligent we are compared with other animals, intuition might dictate that animals are not able to think about the future, as suggested by $\mathrm{S} \& \mathrm{C}$ as well as by others. But foresight is not necessarily an all-or-none phenomenon. Therefore, rather than surrendering to intuition, I propose an alternative whereby foresight is a continuum in that it is present in animals to the extent that it is needed for survival in their own environments. Indeed, if a costly faculty such as foresight developed in humans for survival, it makes sense that nonhumans could benefit from it as well. Until definitive evidence to support the claim that nonhumans lack foresight is potentially reported, this alternative of a continuum is as plausible and thus should be assigned a comparable likelihood, which gives less weight to intuition, during treatments such as this presented by S\&C. In other words, instead of providing complex explanations for behavior that is reminiscent of mental time travel in animals (e.g., "a combination of predispositions and specific learning algorithms"; target article, sect. 3.2, para. 2 ), we can follow the logic of Occam's razor and simply call it foresight of reduced magnitude.

That said, it is easy to see that animals would require foresight that is considerably less complex and rich in scenarios than that required by humans. Consider the range of activities in a life of a nonhuman animal, which is the "pool" of elements that can be combined to create a foresight. This pool includes sleeping, eating, grooming, mating, and usually not much more. In other words, no one but humans is expected to be able to imagine scenarios as complex as sipping a piña colada on the beach while checking e-mail on a laptop connected to the Internet via satellite. As reviewed in the target article, animals nevertheless show meaningful "precursors" of foresight ability. According to the alternative I proposed here, however, instead of a precursor, these manifestations may represent actual foresights, albeit of lower complexity. What might be seen as "limited ability for mental time travel" could indicate that animals have simply evolved as much of this ability as their environment and way of life require.

This issue of an all-or-none versus a continuum is analogous to another important issue. In the context of future-related processes, there is foresight that involves elaborate future-related thought, simulation and planning, and there are the simpler associations (e.g., fire = hot), which can be completely automatic and accomplished outside of awareness (Bar, in press). Are these two processes qualitatively different, relying on independent mechanisms, or do they reflect different extremes on a continuous spectrum of complexity? Although it is not clear if nonhuman animals possess foresight, it is clear that they can use simple predictions that are based on associations (i.e., they learn from 
experience and apply it in the future). Therefore, they might simply lack the presumably other mechanism required for elaborated foresight (Gilbert 2006). On the other hand, one could argue that while simple associations are merely atomic elements, when accumulated, they could serve the basis for phenomena as complex as foresight simulation (Bar et al. 2007). Let us consider a reasonably complex example: You need to get your friend a birthday gift. This does not sound like a simple associative operation. However, when we examine the constituent operations required to achieve this goal, it becomes apparent that experience-based associations may suffice: Your knowledge of this friend's taste as well as needs could be linked in an associative cluster (e.g., a context frame; Bar 2004); the items that correspond to this associative cluster (e.g., your friend likes but does not have an iPod, a vegetarian cookbook, a Toyota FJ Cruiser, 1980s memorabilia) are each associated in your memory with a certain price range and with knowledge of how to get each of these items, and they can be further linked with knowledge about your budget, time constraint, and so on. Therefore, a task that initially seemed quite remote from being based on associations, could be achieved by relying on associations and their appropriate integration. If foresight is indeed derived from associations of varying degree of complexity (which we might not always be aware of), this would support the proposal that foresight could be seen as a continual phenomenon of different levels of complexity and thus possibly present in animals, though to a lower degree than in humans.

The issue of associations is also related to the second part of this commentary. A critical characteristic required from the memory system that subserves foresight is flexibility. This is derived primarily from the notion that in creating foresights we frequently have to rely on past experience while modifying their details to adhere to novel situations. At the same time, it is widely endorsed, and presented logically by S\&C, that episodic memory is the "database" from which foresights are drawn. Episodic memory encodes specific experiences (Tulving 1984; Squire \& Zola 1998) using associative representations (Eichenbaum \& Fortin 2005) and, as S\&C review, contains the particularities of specific episodes (although episodic memory is far from being a perfect depiction of reality; see Schacter \& Addis's commentary in this issue). That episodic memory is crucial for foresight is evident from reports of loss of foresight by patients with a compromised episodic memory, typically in medial-temporal amnesia (Klein et al. 2002b; Hassabis et al. 2007). What begs an explanation is how would a system that is so specific in its representation of relations can at the same time be flexible enough to be applied in novel situations. This paradox emphasizes that more work is needed before we understand the operations done on memory to derive foresight (Bar, in press). Addressing the computational and neural operations used for reconstructing and translating an existing memory into generalized future "memory" (Ingvar 1985) would result in knowledge with implications leading to important clinical, theoretical, and technological advances.

\section{ACKNOWLEDGMENTS}

Thanks to Dan Gilbert. The preparation of this commentary was supported by grants from the NINDS and the McDonnell Foundation.

\section{Is mental time travel a frame-of-reference issue?}

DOI: $10.1017 /$ S0140525X07002014

\author{
Doris Bischof-Köhler and Norbert Bischof
}

Department of Psychology, University of Munich, D-80802 Munich, Germany. bischof@psy.uni-muenchen.de bischof@psy.uni-muenchen.de

http://www.bischof.com

http://www.bischof.com

Abstract: Mental time travel and theory of mind develop, both phyloand ontogenetically, at the same stage. We argue that this synchrony is due to the emergence of a shared competence, namely, the ability to become aware of frames of reference.

As Suddendorf \& Corballis (S\&C) aptly point out, present evidence suggests that mental time travel is a specifically human phenomenon. This holds true, however, for other abilities as well, particularly theory of mind. This presents no problem for the contemporary "modularity" trend, as one can easily imagine any number of competences that are independent and domain-specific but emerge in phylogenetic synchrony, all the same, because they require cooperation in order to become functional. S\&C appear to think along this line as well when they refer to theory of mind among the tools necessary for time travel, although they remain somewhat vague as to exactly how folk psychology contributes to a concept of time. Nonetheless, the assumption that time travel and theory of mind are outcomes of separate mechanisms poses the difficulty that their joint emergence occurs not only in phylogeny but in ontogeny, as well. We were able to show in a sample of 170 children that at around the fourth birthday a set of new competences synchronously develop. They include: a theory of mind as tested in a false-belief paradigm; time comprehension as demonstrated by predicting and comparing the running times of hourglasses; and the abilities to delay gratification, to temporally organize conflicting desires, and to anticipate future needs (Bischof-Köhler 2000). The correlation between these competences is age-independent. It would be difficult, in fact, to identify any vital necessity requiring their joint functioning at such an early age. All this suggests that these competences rest on a common mechanism.

We consider "frame-of-reference awareness" to be that mechanism. The term frame of reference was introduced by Gestalt theorists. Koffka (1935) thoroughly dealt with it under the label "framework" as distinguished from "things." If a feature of a thing is perceived as its absolute property, but is actually relative to its surrounding background, then the latter acts as a frame of reference for the former. Take size, for example. People in an Ames' room appear to grow or shrink when their position is shifted from one corner to the other. This effect is actually due to the background's geometry, but the observer, even if cognizant of the trick, is unable to perceive it this way. Gestaltists have primarily used the frame-of-reference concept to account for perceptual constancy phenomena. Koffka pointed out, though, that it can also be applied to social constructs like "civilization," and Lewin (1946) was among the first to extend it even further to motivational phenomena. Lewin stated that a toy might have a different emotional valence to a child depending on whether or not the mother is present, and explicitly compared this effect to the varying appearance of an object's size or color against different backgrounds. Following this line of thought, we may consider that motivation in general functions as a frame of reference that supplies a mood background organizing the valence profile of life space. Different things become salient, and the world takes on differing physiognomy for a subject when it is hungry, or alarmed, or in love.

Usually, frame-of-reference activity remains inconspicuous or, as Wolfgang Köhler put it, "silent." Only when contradicting frames overlap and cause experiential ambiguities, does one become aware of them. We postulate that the potential for this awareness is beyond the grasp of even the great apes and thus a core constituent of the human condition.

Mental time travel unquestionably requires the ability to reflect upon frames of reference. A shifting of temporal coordinates is necessary in order to conceive of a forthcoming event in the past tense by imagining it from an even further future perspective. Foresight as such, however, does not yet require 
frame-of-reference awareness. What really matters is foresight of need states, that is, of motivational frames of reference other than the ones shaping the present world view. But why should it be necessary to imagine non-present need states in the first place? The answer leads to the core assumption of the Bischof-Köhler hypothesis. It deals with the problem of motive priority management. Animals, when experiencing contradicting needs, cannot but follow the strongest need and suppress the others. Humans may still behave the same way, but they have access to a higher level of behavioral organization. They are able to conceive of a temporal axis analogous to space that provides a buffer on which competing desires, including future ones, may be shifted, postponed, adjourned, delayed, or anticipated, according to their expected consummatory value and success, thus overriding the imperative of instant gratification. The advantage of such an arrangement is obvious. The use of fire, for example, would not have been practicable for our ancestors had they not been able to collect firewood well before commencing to freeze.

Obviously, theory of mind can also be interpreted as frame-ofreference awareness. Understanding false belief, as well as Flavell's level II perspective taking, depend on the ability to simultaneously represent contradicting frames of reference. Rephrasing theory of mind in such a way goes beyond describing the same issue in different theoretical languages. Usually the selective advantage of theory of mind is seen as an improvement of social-cognitive folk psychology. However, if we conceive of it as frame-of-reference awareness instead, we describe it as a multipurpose "module" whose adaptive function is much more general. Looked at in this way, mental time travel and theory of mind need not be externally arranged in a means-end relation, but appear as different outcomes of one and the same competence.

\section{The costs of mental time travel}

\section{DOI: $10.1017 /$ S0140525X07002026}

\section{Martin Brüne and Ute Brüne-Cohrs \\ Centre for Psychiatry, Psychotherapy, Psychosomatics and Preventative Medicine, University of Bochum, 44791 Bochum, Germany. \\ Martin.Bruene@rub.de Ute.Bruene-Cohrs@rub.de}

Abstract: A species like ours, whose life critically depends on the ability to foresee, plan, and shape future events, is vulnerable to dysfunction if any one facet contributing to what Suddendorf \& Corballis $(\mathrm{S} \& \mathrm{C})$ call "mental time travel" (MTT) is affected by disease. Although the authors mention brain pathology as a potential cause of disturbed MTT, they fail to explore psychopathological syndromes as a source to better understand the significance of MTT for normal functioning and adaptive behaviour.

The ability to foresee and shape future events using episodic memory as a source of information that facilitates action planning has been termed "mental time travel" (MTT). Suddendorf \& Corballis $(\mathrm{S} \& \mathrm{C})$ convincingly argue in favour of an adaptive value of such a cognitive capacity compared to fixed or instinctual ways of predicting future scenarios, as in hibernators. It is argued that MTT emerged at some point during human evolution to predict future actions of conspecifics. In other words, MTT probably evolved because selection favoured individuals who had the capacity to flexibly use MTT over others who were less able to create mental models of future events, including social interactions. S\&C reason that MTT could be specific to humans, because there is little evidence for MTT in nonhuman animals. However, the authors also emphasise that testing MTT in nonhuman animals is a difficult if not impossible enterprise, partly due to the absence of verbal reports of episodic memory contents in animals.
In light of the central claim of the target article that MTT is human-specific, it is surprising that the authors fail to make use of insights from psychopathology. The possibility that evolutionary psychology and psychopathology can mutually inform each other has been outlined by one of the leading ethologists of the 20th century, Konrad Lorenz - notably in a footnote of his formerly acclaimed, but nowadays largely disregarded, book Behind the Mirror, first published in German in 1973. (Lorenz developed many of his ideas much earlier, during his time as a prisoner of war in the former Soviet Union, recently rediscovered and published as The Russian Manuscript: Lorenz 1992.) Basically, Lorenz, who was a psychiatrist himself, pointed out that analysing failures of any given behaviour might contribute more to the understanding of its physiology than studying the intact physiology itself. Although not explicitly stated, cognitive and emotional correlates of behaviour could be examined in the same way (Lorenz 1973/1977).

We therefore propose that any attempt to dissect the cognitive architecture of MTT would greatly benefit from a careful consideration of certain psychopathological conditions, where one or more aspect of MTT is arguably affected by malfunctioning.

S\&C define the "ingredients" of human MTT using a theatre production as a metaphor. Accordingly, imagination, self-awareness, recursion, metarepresentation, appreciation of the time dimension (including temporal distance, order, and location within time patterns), executive functioning, and prospective memory are considered key components of MTT, with episodic memory lying at the very core. Hence, mental disorders, which are characterised by the dysfunction of one or more of the key components of MTT, may not only help elucidate the neuropsychology, but also the underlying neural networks involved in MTT.

The most devastating disorders with putative deficits in MTT are probably the various forms of dementia. S\&C briefly mention amnesia and damage to the frontal lobes as potential causes of disrupted MTT, without going into detail, however, about what exactly the deficits could be at both the neurotransmitter level and brain pathology. In Alzheimer's disease (AD), for example, the loss of episodic and prospective memory is profound, even in preclinical stages (Jones et al. 2006), and this functional deterioration is associated with a progressive cholinergic deficit commencing in the nucleus basalis of Meynert. It is well known that the entorhinal cortex and the hippocampus suffer a severe loss of function through the accumulation of neuritic plaques and tangles. In contrast, it is only quite recently that researchers have discovered that the so-called spindle cells - an evolutionarily novel cell type unique to apes and humans, located in the anterior cingulate cortex where the integration of cognition, emotion, and motor control takes place - degenerate early in the course of AD (Nimchinsky et al. 1995).

Similarly, in full-blown Korsakoff's syndrome - which may develop in conditions associated with a severe deficiency of vitamin B1 (in Western societies, most often chronic alcoholism) episodic memory deficits may lead to a complete loss of personal history. Even more pronounced than in AD, patients with Korsakoff's syndrome "make up" their personal history, referred to as producing confabulations. These patients are unaware of the incorrectness of their "memories." A striking feature of this disorder is that patients with Korsakoff's syndrome may invent a totally different story when asked the same question only minutes later. Thus, although the patients' imagination is apparently left intact, they are confined to the present, and this might be one reason for the conspicuousness of Korsakoff's syndrome compared to other forms of dementia (Sacks 1985). The anatomical lesion of Korsakoff's syndrome is primarily located in the corpora mamillaria.

Comparing deficits in episodic memory in AD patients to those in different types of frontotemporal lobe degeneration (FTDL) 
may help address the issue of the relevance of each specific component of MTT. AD patients typically show a temporally graded memory loss with more remote memories being better preserved than more recent ones, whereas patients with semantic dementia (SD) involving the anterior temporal pole show the reversed gradient. However, patients with a frontal variant of FTD ( $\mathrm{fv}-\mathrm{FTD}$ ) do not show any temporal gradient of episodic memory deficits (Piolino et al. 2003). Here, the altered memory retrieval as well as executive dysfunction seem to contribute to deficits in episodic memory, which is in support of the components relevant for MTT.

The behavioural consequences of both AD and Korsakoff's syndrome are that, depending on the severity of the disorder, patients are unable to lead independent lives - partly, because their toolboxes that allow the flexible use of a vocabulary to construct future events and to refer to autobiographical memories in a meaningful personal way are irreversibly destroyed.

Interestingly, another evolutionary cost may result from the hyper-functioning of MTT. Patients with obsessive-compulsive disorder (OCD) suffer from intrusive thoughts to which they often over-ascribe meaning in order to prevent future (negative) events, perhaps as a consequence of the tendency to excessively reflect upon their cognitive processes (Janeck et al. 2003). This often precludes effective future action planning in that patients with OCD are caught in repetitive behaviours to avoid erroneously anticipated threat or danger. Notably, those brain regions involved in future action planning, as well as episodic and prospective memory retrieval, including the ACC, the prefrontal cortex, and the dorsolateral prefrontal cortex, have been found overactive in patients with OCD; and this could arguably be interpreted as an MTT overshoot (Brüne 2006). Here, serotonin and dopamine are probably the key players in the pathology of OCD.

These examples of failures of MTT in mental disorders certainly do not represent an exhaustive list. Schizophrenia, for instance, can be seen as another prime example, where virtually any one of the ingredients of MTT may be disrupted, including metarepresentation or "theory of mind" (Brüne \& Brüne-Cohrs 2006) and time consciousness (Vogeley \& Kupke 2007).

In any event, the purpose of this commentary is to pinpoint the need for more interdisciplinary approaches to the understanding of the evolved psychology of our species, to which the study of psychopathology - particularly of human-specific domains - can contribute a lot more than is currently conceived.

\section{Prospection and the brain}

\section{DOI: $10.1017 /$ S0140525X07002038}

\section{Randy L. Buckner \\ Department of Psychology, Center for Brain Science, Harvard University, Athinoula A Martinos Center, Massachusetts General Hospital, Howard Hughes Medical Institute, Cambridge, MA 02138. \\ rbuckner@wjh.harvard.edu}

\begin{abstract}
Suddendorf \& Corballis $(\mathrm{S} \& \mathrm{C})$ propose that the capacity to flexibly forsee the future was a critical step in human evolution and is accomplished by a set of component processes that can be likened to a theater production. Understanding the brain-bases of these functions may help to clarify the hypothesized component processes, inform us of how and when they are used adaptively, and also provide empirical ways of exploring to what degree these abilities exist and are implemented similarly (or differently) across species.
\end{abstract}

The notion that a major adaptive function of human cognition is our ability to imagine the future has been rumbling for some time. Ideas about brain processes directed toward future thinking - often called prospection - have emerged from early studies using brain imaging (Ingvar 1979; 1985), work on human memory (Tulving 1985; 2005), and theories in social cognition (Gilbert 2006; Klein et al. 2002a). These rumblings have erupted over the past year (for reviews, see Buckner \& Carroll 2007; Schacter \& Addis 2007a). Recent empirical observations using brain imaging reveal that frontal and medial temporal memory systems participate in envisioning the future in ways that parallel their role in remembering the past (Addis et al. 2007; Okuda et al. 2003; Szpunar et al. 2007). Characterization of the deficits in patients with brain lesions shows that amnesia is linked to impairments of prospection and imagination (Klein et al. 2002a; Hassabis et al. 2007).

The central idea emerging from this recent flurry of research activity is that humans possess a brain system, or interaction among brain systems, which functions to construct mental simulations that represent possible upcoming situations - a form of "life simulator." While forms of these abilities exist in other animal species, the capacity for mental exploration of future possibilities is extraordinarily well developed in humans. Planning, social cognition, remembering, moral reasoning, and even daydreaming may depend on these brain systems (Buckner \& Carroll 2007).

Building upon their earlier seminal work (Suddendorf \& Corballis 1997), the target authors Suddendorf \& Corballis $(\mathrm{S} \& \mathrm{C})$ again return to the question of what foresight is and whether it is unique to humans. This is a well-timed review. Much of its value is to renew attention and revisit some of the central ideas raised in their earlier work. A central message is that, as a field, we should consider the adaptive functions of capacities such as episodic memory (mental time travel) in the context of how they aid decisions and future-oriented behavior. A lingering debate is to what degree other animals possess these capacities that are so well developed in humans. In a recent opinion piece, Buckner and Carroll (2007) concluded that (1) certain animal species such as the scrub jay have adapted to overcome similar challenges that prospection has emerged to benefit in human; (2) prospection and related abilities are more developed in humans than in other animals, perhaps with qualitative differences that emerge from our self-awareness; and (3) other animals possess behavioral and neural patterns that might represent proto-forms of prospection and related abilities. I do not repeat the arguments here.

What I do want to address is whether we are beginning to stall in our progress by only exploring the question at the behavioral level - something that can be considered a weakness (or an incompleteness) in S\&C's present argument. They make an explicit separation between their ideas and hypotheses about neural implementation. When introducing the pursuit of their theory of components of mental time travel, they write that no claims are made "about simulation or any other mechanisms by which this may be instantiated in the brain" (sect. 4, para. 2). The target article discusses brain evidence in the section on "neurophysiological evidence" (sect. 2.3), so it is clear that brain data are seen as relevant. Nonetheless, the developed framework proceeds independent of information from studies of the brain.

This orientation has two limitations. The first limitation is that an opportunity is missed by not providing an integrated brainbehavior discussion, because the present theory does not help conceptualize much of the emerging empirical data on the neural implementation of prospection including the important roles of frontal and medial temporal systems. The second, more poignant, limitation is that information about neural architecture will almost certainly help us to understand both how the capabilities are implemented in the human and also to what degree the underlying processes are similar or different across species. The theater metaphor in the target article and reframing of Tulving's models of memory into contemporary futureoriented versions are extremely useful frameworks for generating ideas about the components involved in flexible future-oriented human cognition. But how can we test that these processes really exist? And, relevant to the question of comparative 
cognition, how are we to explore whether similar mechanisms are at work in other animals? I suspect the answer will come in pairing cognitive and behavioral theories with directed exploration of their implementation within the brain.

Specifically, in the target article a novel framework for understanding component processes involved in prospection is proposed using a theater metaphor. Within the theater metaphor a critical component process is the "stage" - a representational space whereby simulations of the future can be constructed and explored. A proposed detail of this constructional space is that it allows a representation of current reality and also secondary representations that explore future possibilities. This intriguing hypothesis implicitly specifies a mechanism of representation that can be informed by underlying neural implementation.

Neural investigation may be particularly relevant to explore similarities and differences in how information is represented across species. For example, it may be possible to identify neural sequences, in particular in the hippocampus of behaving rats, that "preplay" upcoming decision choices (such as left turns in a maze, versus right turns). One could then ask to what degree such representations are adaptive in rats, how they are similar or different from the kinds of representations observed in humans, and what kinds of limitations arise from any observed differences. Rats are mentioned here as an example because paradigms that probe representation in rats are particularly well developed experimentally (e.g., Ferbinteanu \& Shapiro 2003; Foster \& Wilson 2006). The same approach could be applied to other nonhuman species, barring the great apes. It will be particularly interesting to understand neural implementation of convergent behaviors in distant animal species, such the recent work on scrub jays. I suspect we will find significant differences in implementation. From these differences we may learn about the power of natural selection to find convergent paths to complex neural processes much in the same way wings for flight and fins for swimming have evolved on multiple occasions. Put simply, understanding neural implementation will almost certainly help to clarify the component processes used by humans for prospection and their relations to other species.

\section{A unique role for the hippocampus in recollecting the past and remembering the future}

DOI: 10.1017/S0140525X0700204X

\author{
Valerie A. Carr ${ }^{\mathrm{a}}$ and Indre V. Viskontas ${ }^{\mathrm{b}}$ \\ a Interdepartmental Program in Neuroscience, University of California Los \\ Angeles, Los Angeles, CA 90095-1563; ${ }^{\mathrm{b}}$ Memory \& Aging Center, \\ Department of Neurology, University of California San Francisco, San \\ Francisco, CA 94143. \\ valeriec@ucla.edu iviskontas@memory.ucsf.edu
}

Abstract: Suddendorf \& Corballis (S\&C) argue that episodic memory is the most flexible and recently evolved memory system, and point to the reorganization of prefrontal cortex throughout human evolution as the neuroanatomical substrate. Their approach, however, fails to address the unique role that the hippocampus, a primitive brain region, plays in creating and recalling episodic memories, as well as future event construction.

Although Suddendorf \& Corballis (S\&C) provide a thoughtprovoking framework for the cognitive processes involved in remembering the past and imagining the future, their discussion of the neural mechanisms underlying these processes fails to adequately acknowledge the important role of the medial temporal lobe (MTL) in episodic memory. The authors argue that episodic memory is the most flexible of the memory-based neural systems and also the most recently evolved, pointing to the expansion and reorganization of the prefrontal cortex throughout evolution as the driving force behind the emergence of episodic memory in humans. By limiting their discussion of neural mechanisms to the prefrontal cortex, the authors ignore the role that a much more primitive region of the brain plays in episodic memory: the hippocampus. The MTL, and in particular the hippocampus, has been repeatedly implicated in the encoding and retrieval of episodic memories (for review, see Davachi 2006), and recent evidence indicates that this region may also play an important role in future event construction (Addis et al. 2007; Hassabis et al. 2007; Okuda et al. 2003).

As is now well-established, the MTL is anatomically suited to bind information from different sensory cortices into a cohesive trace: the hippocampus, via the entorhinal cortex, receives convergent input from a variety of unimodal and polymodal association cortices in the temporal, frontal, and parietal lobes (Bunsey \& Eichenbaum 1995; Suzuki \& Amaral 1994). Several neurocomputational models demonstrate how the unique anatomy and physiology of the hippocampus enables the creation of specific, detailed memories. O'Reilly and Rudy (2001) proposed the Complementary Learning Systems (CLS) model to differentiate between representations serving episodic recollection and those serving familiarity. According to the CLS model, the hippocampus is specialized for rapidly storing episodic memories, whereas the surrounding neocortex stores information about the statistical regularities in the world. As in other models, the CLS model supposes that the hippocampus uses sparse coding to store distinct, pattern-separated representations of elements, thus avoiding catastrophic interference, or overlapping representations. The neocortex, on the contrary, assigns similar patterns of activation to similar stimuli, thereby representing the shared structure of events via overlapping representations, and generalizing to novel stimuli when they share features of previously encountered stimuli.

During encoding, synapses in the perforant path projecting from the entorhinal cortex to the dentate gyrus, CA3 and CA1 fields, undergo self-organization by creating increasingly sparser representations of inputs. Meanwhile, excitatory synapses in the CA1 and CA3 regions associate these sparse representations, forming memory traces (Hasselmo et al. 1996). During retrieval, a partial pattern of previously studied material is presented to the hippocampal network. Upon reaching CA3, the partial input pattern is completed via strengthened weights in the extensive recurrent collateral network. The complete CA3 representation then activates the associated CA1 representation, which, via the subiculum, activates the original entorhinal pattern (O’Reilly \& McClelland 1994; Rolls \& Kesner 2006). This activation, in turn, serves to trigger reinstatement of activity in the neocortex reflecting the multi-modal content of the original memory.

Functional neuroimaging studies have demonstrated a selective role for the hippocampus in both encoding (Uncapher \& Rugg 2005) and retrieving (Eldridge et al. 2000; Woodruff et al. 2005) episodic memories. In an effort to determine activation patterns at the subregional level, Eldridge et al. (2005) scanned participants using high-resolution functional magnetic resonance imaging (fMRI) of the MTL during both encoding and retrieval. Results show that the dentate gyrus and CA3 field were selectively active during episodic memory formation, whereas the subiculum was active during retrieval of the study episode.

Data from single-unit recordings in humans also support the idea that the hippocampus relies on sparse coding to function efficiently. Viskontas et al. (2006) examined recognition memory in a group of epilepsy patients undergoing pre-surgical mapping. Patients viewed novel images and were asked to make old/new judgments for each presentation. Upon the second presentation of a given stimulus, a large subset of hippocampal neurons showed a decrease from baseline firing, while a 
small subset showed excitation. These excitatory responses have been shown to be specific for particular items (Quiroga et al. 2005). Inhibition of firing to previously seen stimuli may serve to increase signal-to-noise ratio, thereby supporting sparse coding and effective mnemonic processing. Furthermore, preliminary data suggest that some cells in the hippocampus show excitatory responses to a particular item only when that item is encountered alone or in the context in which it was originally encountered (Viskontas 2006).

More recently, evidence has emerged supporting a role for the MTL in imagining future experiences. In a positron emission tomography (PET) study, Okuda et al. (2003) found greater or equivalent levels of activation while subjects spoke about future prospects versus past experiences in the right hippocampus and bilateral parahippocampal cortices. Similar results were reported in a fMRI study comparing past and future event elaboration, in which the left hippocampus and bilateral parahippocampal cortices were commonly engaged by both tasks (Addis et al. 2007). Interestingly, the authors also report that construction of future versus past events uniquely activated the right hippocampus. Neuroimaging results are supported by reports of marked impairments in imagining future events in patients with hippocampal amnesia (Hassabis et al. 2007).

As the aforementioned neurocomputational, single-unit recording, functional MRI, PET, and patient studies suggest, there is a wealth of evidence supporting a role for the hippocampus in both episodic memory and in envisioning future experiences. Although we do not deny that the prefrontal cortex plays an important and complementary role in these processes, we find that $\mathrm{S} \& \mathrm{C}$ rely too heavily on the recent evolution of this region in humans in arguing against mental time travel in other species. In fact, a recent study of scrub jay behavior suggests that these birds are, in fact, capable of future planning without reference to their current motivational state, or fixed action patterns, challenging the notion that future planning requires a prefrontal cortex (Raby et al. 2007). The hippocampus is phylogenetically among the oldest parts of the brain, and may therefore allow nonhuman animals to project both backwards in time and forwards into the future. Thus, as increasingly innovative nonverbal tests of episodic memory develop, we may be surprised to discover the time-traveling capabilities of a variety of species.

\section{Emotional aspects of mental time travel}

DOI: $10.1017 / S 0140525 X 07002051$

\section{Arnaud D'Argembeau ${ }^{\mathrm{a}}$ and Martial Van der Linden ${ }^{\mathrm{b}}$ \\ ${ }^{a}$ Cognitive Psychopathology Unit, University of Liège, 4000 Liège, Belgium; ${ }^{\mathrm{b}}$ Cognitive Psychopathology and Neuropsychology Unit, University of Geneva, 1205 Geneva, Switzerland. \\ a.dargembeau@ulg.ac.be http://www.ppc.ulg.ac.be \\ Martial.VanDerLinden@pse.unige.ch \\ http://www.unige.ch/fapse/PSY/persons/vanderlinden/index.html}

\begin{abstract}
We consider three possible reasons why humans might accord a privileged status to emotional information when mentally traveling backward or forward in time. First, mental simulation of emotional situations helps one to make adaptive decisions. Second, it can serve an emotion regulation function. Third, it helps people to construct and maintain a positive view of the self.
\end{abstract}

Suddendorf \& Corballis (S\&C) convincingly argue that memories of past events and images of future ones are not literal representations of the past and future, but are instead the products of generative, constructive processes that (re)create mental representations by (re)arranging pieces of information retrieved from memory (see also Schacter \& Addis 2007a). The resulting representations can therefore be highly selective, and the pieces of information that are encoded, maintained, and retrieved from memory in priority are those that are most relevant to an individual's goals, beliefs, and concerns (Conway 2005). Emotion plays an important role in this selection process, as it signals the occurrence of information that has potentially important implications with regard to goals (Ellsworth \& Scherer 2003). Consequently, emotional stimuli are typically remembered with more details than neutral stimuli (D'Argembeau \& Van der Linden 2005; Kensinger et al. 2006), and autobiographical memories for emotional events are associated with a higher subjective feeling of mentally reliving the past (D'Argembeau et al. 2003; Talarico et al. 2004). In addition, for both past and future, representations of positive events are associated with a greater feeling of reexperiencing (or pre-experiencing) than representations of negative events (D’Argembeau \& Van der Linden 2004). In this commentary, we would therefore like to suggest that humans accord a privileged status to emotional information when they mentally travel backward or forward in time, and we consider some reasons why this might be the case.

$\mathrm{S} \& \mathrm{C}$ argue that the primary function of mental time travel is to enhance biological fitness in the future: Mentally simulating various versions of the future, and their respective consequences, enables one to act flexibly in the present to increase one's future survival chances. We completely agree, and would add that the affective charge of the generated mental images is a key element in this respect, the driving force that guides our current decisions. Affective states associated with mental simulations of positive and negative outcomes motivate us to engage in certain types of behaviors (e.g., start a diet) and to avoid others (e.g., quit smoking), in order to maximize the probability of attaining our goals. Future rewards and punishments need not be consciously represented to guide decision making (Bechara \& Damasio 2005), but the conscious mental simulation of emotional situations, through mental time travel, undoubtedly provides unique information that promotes successful adaptation to life circumstances. Negative memories remind people of their past errors and provide cues on how to avoid undesired outcomes or minimize their consequences, whereas positive memories remind people of their past accomplishments and provide cues on how to attain success. These pieces of information regarding the personal past can be used to generate representations of the future that specify (a) which situations should be approached or avoided, and (b) how to maximize the probability of attaining/ avoiding them. Mental imagery, especially visual imagery, may play a particularly important role in representing such goal-related information, as it is a sort of "language" of goals (Conway et al. 2004). Interestingly, it has been found that people who have higher visual imagery capacities generate more detailed representations of their personal past and future, and rate their representations of future events as being more emotional and meaningful (D’Argembeau \& Van der Linden 2006). It would be interesting to investigate whether these individual differences are correlated with the ability to make adaptive decisions. Alternatively, the ability to envision detailed emotional past and future events should be explored in individuals who show impairments in judgment and decision-making in real-life settings, such as patients with lesions of the ventromedial prefrontal cortex (see Bechara \& Damasio 2005).

Although the primary function of emotional aspects of mental time travel may be to help one make adaptive decisions, mental representations of emotional episodes probably serve other functions as well. Representations of emotional events induce significant modifications of emotional responses and feelings (Damasio et al. 2000), and may therefore be used to regulate affective states. Sometimes we remember or imagine positive experiences, not so much to help us make decisions or plan future actions, but simply to feel better in the present. There is evidence that people occasionally retrieve positive events in order to repair a negative mood (McFarland \& Buehler 1998). Note that the use of mental 
simulations to regulate mood states is not always straightforward, however; people probably use rather complex strategies that may vary across situations. For example, one may strive to generate or prolong a positive affective state by envisioning negative events (e.g., how things might be worse), which allows one to feel good in comparison (Sanna 2000).

Finally, a third function of the emotional aspects of mental time travel may be to provide material to support representations of the self. Most people hold positive views of themselves and are more willing to consider information that bolsters their selfimage than information that contradicts it (Baumeister 1998). Thus, memory tends to be biased toward confirming positive self-views. For example, as mentioned earlier, positive episodes are subjectively experienced with more details and with stronger feelings of (re)living than negative episodes, for both the past and the future (D’Argembeau \& Van der Linden 2004). In addition, when asked to think about their future, people spontaneously imagine more positive than negative events (Newby-Clark \& Ross 2003) and judge positive events as being more likely to happen (Weinstein 1980). The importance of the balance between positive and negative future thinking is further revealed by its disturbance in certain psychopathological conditions. For example, depressed individuals tend to generate fewer positive future events, while anxious individuals tend to generate more negative future experiences (MacLeod \& Byrne 1996). Positive biases in representing the past and future probably help maintain a positive view of the self and foster optimism concerning one's personal future, which may promote physical and mental health (Taylor \& Brown 1988).

\section{ACKNOWLEDGMENTS}

Arnaud D'Argembeau is a postdoctoral researcher for the Belgian National Fund for Scientific Research (F.N.R.S.). This work was supported by a grant from the French-speaking community of Belgium (ARC, Convention 06/11-340).

\section{Storing events to retell them}

DOI: $10.1017 /$ S0140525X07002063

\section{Jean-Louis Dessalles \\ ParisTech, Ecole Nationale Superieure des Telecommunications, F-75013 Paris, France. \\ dessalles@enst.fr www.enst.fr/ jld}

Abstract: Episodic memory is certainly a unique endowment, but its primary purpose is something other than to provide raw material for creative synthesis of future scenarios. Remembered episodes are exactly those that are worth telling. The function of episodic memory, in my view, is to accumulate stories that are relevant to recount in conversation.

As the authors of the target article suggest, episodic memory (EM) can be seen as a "plug-in" device added to a standard vertebrate brain, and quite an expensive one, as much of our cortical mass seems devoted to it. Suddendorf \& Corballis (S\&C) are certainly right to say that it demands an evolutionary explanation. Their suggestion is that EM serves only the practical purpose of providing raw material for future planning. In a similar attempt to provide an evolutionary account for EM, Brown and Kulik (1977) highlighted the benefit of storing unexpected and highly emotional events, as "a marked departure from the ordinary in a consequential domain would leave [the individual] unprepared to respond adequately and endanger his survival" (p. 97). The problem with such accounts is that EM is badly designed for its alleged function.

Does an optimal use of storage capacity leave room for the memory of instantiated episodes? In machine learning, rote learning is an inefficient strategy. The purpose of any learning task is to make generalization possible. A good way to perform induction is to aggregate experience into structures such as prototypes or clusters. Storing particular instances (e.g., cluster centers) generally makes sense if they are statistically representative. This function is implemented in living beings through semantic and procedural memory.

To delineate categories, it may be useful to remember borderline instances, as with support vectors (Cornuejols \& Miclet 2002). Also, in certain applications in which data are scarce and non-homogeneous, storing actual encountered examples, regardless of their representativeness, may be a viable strategy. The Case-Based Reasoning technique (Kolodner 1993) aims at solving new problems by matching them with memorized known examples. Superficially, episodic memory could be understood as a biological implementation of these principles, but its actual form does not match up to the assignment.

Episodic memory is highly selective. It retains a tiny fraction of all our daily experiences. One may come across dozens of people each day and remember only a few encounters per month. Selected episodes are, however, retained with a significant amount of detail, including what S\&C call the www criterion. From an efficiency perspective, details such as the precise location in space and time, the weather conditions, the persons present, the words exchanged, and so forth, are most often irrelevant and yet are almost systematically remembered, even in the long term in cases when emotion is high (Brown \& Kulik 1977). From a computational perspective, not only do such details represent a waste of storage, they also hinder and mislead retrieval matching.

An alternative view is that EM is an outgrowth of the language faculty (Dessalles 2006). It is not fortuitous that memorized episodes are exactly those which are narratable. People spend one fifth of their waking time in spontaneous conversation (Dunbar 1998), and a significant share of this time is devoted to reporting past events (Tannen 1984, p. 99; Eggins \& Slade 1997, p. 265). Interlocutors draw from their memory relevant episodes that they can relate to the current conversational topic and they systematically try to recount them. However, only a tiny fraction of past experiences may be recounted in this way. One crucial requirement is that reported stories must appear unexpected (Dessalles, in press).

The requirement of unexpectedness provides also a good prediction of the kind of episodes that are preferentially stored in memory. To appear unexpected, a situation must be less complex (i.e., more easily describable) than expected (Dessalles, in press). Witnessing a six-legged cow makes both a memorable event and a good story to tell, just because this cow, thanks to its unique peculiarity, requires a minimal description to be distinguished from all others. If, as we claim, the primary purpose of storing episodes is to offer material for future recounting, then systematically remembering details such as time and space location makes perfect sense. If the six-legged animal lives in the vicinity, interest is raised. Not specifying the location would leave the listener with the idea that that location requires a lengthy description, and interest drops down. By computing complexity differences, one can derive the way interest varies according to location and time, and according to various factors such as the persons involved (Dessalles, in press). For instance, the interest of coincidentally encountering someone increases with the remoteness (and thus complexity) of the place and with the simplicity of that person, if she happens to be a celebrity or a close acquaintance. It is thus crucial, when memorizing an episode, to remember every detail that may affect the cognitive complexity of the situation.

It may seem surprising that the expensive resources devoted to EM serve such a futile purpose as everyday chatter. This is only because one fails to see that casual conversation is an arena where much of our social existence is decided (Dessalles 2007). Eliciting interest through conversational stories is a high-stakes game. Boring participants are rapidly ignored and may lose their friends. When it comes to establishing solidarity bonds, 
individuals prefer those who successfully demonstrate their informational capacity and their experience with unexpected events. In our species, those who know first or who can draw highly relevant events from their past experiences make potentially good allies. Natural selection favored not only this preference, but also the narrative skills that allow any of us to display these qualities (Dessalles 2007). Episodic memory, in this context, is a crucial tool that enables us to produce the most relevant story at the right time. It has been tailored for this purpose, as demonstrated by the fact that the factors that favor memorization, such as unexpectedness and atypicality (Shapiro \& Fox 2002; Stangor \& McMillan 1992; Woll \& Graesser 1982), are exactly the factors which increase tellability.

This account explains why remembered episodes are communicated, instead of remaining private; why they remain coherent in memory (instead of being dismantled for creative synthesis of future scenarios); why they systematically involve various details and precision; why we keep on memorizing episodes throughout our entire life; and why even slight failures in episodic memory (as those that occur with aging or in certain pathologies) have dramatic influence on social relations. It also explains the uniqueness of EM, which was not selected for increasing planning efficiency, but as a tool in support of language performance.

\section{Mental time travel in the rat: Dissociation of recall and familiarity}

DOI: $10.1017 /$ S0140525X07002075

\section{Madeline J. Eacott and Alexander Easton \\ Department of Psychology, University of Durham, Durham DH1 3LE, United Kingdom. \\ m.j.eacott@durham.ac.uk \\ http://www.dur.ac.uk/behavioural.neuroscience/ \\ alexander.easton@durham.ac.uk \\ http://www.dur.ac.uk/behavioural.neuroscience/}

Abstract: We examine and reject the claim that the past-directed aspect of mental time travel (episodic memory) is unique to humans. Recent work in our laboratory with rats has demonstrated behaviours that resemble "remember, know" judgements about past occasions. Similar to human episodic memory, we can also demonstrate a dissociation in the neural basis of recollection and familiarity in nonhumans.

Suddendorf \& Corballis (S\&C) argue that humans are unique in possessing the ability to mentally travel in time. Episodic memory allows us to mentally travel into the past, and so, it is claimed, nonhuman animals also lack episodic memory. In making this claim, it is important to be clear about one's definition of episodic memory. S\&C write that episodic memory is "about reconstructing particularities of specific events that have happened to the individual" (sect 2.1, emphasis in original). But what particularities might be relevant to this claim? S\&C discuss the widespread claim in the literature that at least a precursor of episodic memory may be found nonhuman animal's memory for the what, where, and when of an event.

Yet S\&C reject evidence of such episodic-like memory from nonhuman animals on two bases. First, they criticise examples of episodic-like memory in nonhuman animals that depend on coding when an event happened, which may, they claim, depend on other cues such as trace strength. Second, they claim that this type of memory could be known rather than remembered. We address these claims using examples of work with rats from our laboratory.

First, we consider what "particularities of specific events that have happened to the individual" episodic-like memory might represent. The problematic claim is that nonhuman animals might code when (or, strictly speaking, how long ago) a remembered event occurred. Yet human episodic memory rarely codes when, and even with all the advantages of verbal labels to help code such information (e.g., "last Wednesday," "19th March"), we are extremely poor at such memory (Friedman 1993). Nevertheless there is evidence that even without such labels, nonhuman animals may code this type of information, although as $\mathrm{S} \& \mathrm{C}$ point out, the difficulty remains of ensuring that trace strength itself cannot provide a sufficient cue without recourse to mental time travel. Yet, could this difficulty be overcome if we asked nonhuman animals about when a remembered event happened in a different way? When we code when something happened, we rarely code the exact time point; instead, we often use convenient contextual cues. For example, I may remember that I mislaid my car keys on Wednesday, but only because of the contextual cue that it happened in the lecture theatre in which I teach only on Wednesday mornings; and I may also differentiate this from a similar event that happened in the afternoon in a different room.

Recently in our lab we have used such contextual information to cue animals to one of two recently experienced events (Eacott et al. 2005; Easton et al. 2006). Rats experience two highly similar events, finding two different objects each day hidden within an E-shaped maze. In the first event each day, object A is found in the left arm of the maze, while object B is in the right arm with a particular visuospatial context present in the maze. In the second event each day, the position of these objects is reversed in the presence of a second visuospatial context. The visuospatial context serves as a contextual cue, like the lecture theatre in the previous example. After the rats have experienced the two events, one of the objects (e.g., object A) is overexposed, giving the rats a natural preference for seeking out the other, nonexposed object (in this case, object B). We then ask whether rats can use their memory to seek out a preferred object when returned to the maze with one of the previous contexts present. The context serves in place of a verbal label for the particular occasion being asked about, so this provides a way of asking the rat "where was object B when you saw it in the black context?" Intact rats efficiently "answer" such a question by seeking out object B (Eacott et al. 2005), and it cannot be claimed that such ability can be reduced to a time-dependent process such as trace decay.

However, S\&C cite this work and claim that, like other examples of memory for the peculiarities of events, this type of memory "may be known rather than remembered" (sect. 3.1, para. 5). Yet how can one address such a criticism? The essential difference between remembering and knowing about a past, personally experienced event lies in the subjective feeling associated with a memory, and clearly this cannot be investigated in nonverbal animals. However, in humans the subjective feelings of knowing and remembering are associated with different neural systems and different patterns of performance when analysing receiver operating curve (ROC) curves (Yonelinas 1994; Yonelinas et al. 1998). Recently we have produced direct behavioural evidence (Easton et al. 2006) of this dissociation in rats. In the task described earlier, intact rats were capable of answering a question equivalent to "where was object B when you saw it in the black context?" by seeking out a hidden object in the appropriate context. Because the object was hidden, this ability must rely on recall, and not recognition memory. Moreover, this recall ability is dependent on the fornix, the main efferent of the hippocampus (Easton et al. 2006). Yet the same fornix-transected rats that could not seek out the preferred object on the basis of recall, showed entirely normal recognition memory of the same objects in the same trials of the task. As these animals had impaired recall, we can assume that their intact recognition ability was mediated by intact familiarity processes. Thus, by using behavioural tasks in conjunction with lesion-based evidence, we can dissociate recall ("remembering") from familiarity-based recognition ("knowing"). This behavioural evidence supports other recent work in rats performing a food-rewarded, old-new, odour recognition paradigm in which statistical analysis of ROC curves show recollection, and familiarity can be dissociated from one another with lesions of the hippocampus specifically impairing recollection (Fortin et al. 2004). 
Of course, we cannot say with any confidence that this "recall" in a rat was associated with the phenomenological experience that accompanies recall in humans. Yet, as S\&C point out, there is as yet no agreement as to how to study such subjective phenomena in humans, and so we should not set the evidential bar for demonstrating recall in rats so high that it cannot be satisfied even for other humans. Nevertheless, by showing increasing evidence for similarity between phenomena in rats and in humans, we can at least claim that we have demonstrated a dissociation between "familiarity-like" memory and "recall-like" memory in the rat.

\section{The meaning of "time" in episodic memory and mental time travel}

DOI: $10.1017 / \mathrm{S} 0140525 X 07002087$

\section{William J. Friedman \\ Department of Psychology, Oberlin College, Oberlin, $\mathrm{OH} 44074$. friedman@oberlin.edu}

\begin{abstract}
The role of time in episodic memory and mental time travel is considered in light of findings on humans' temporal memory and anticipation. Time is not integral or uniform in memory for the past or anticipation of the future. The commonalities of episodic memory and anticipation require further study.
\end{abstract}

Temporal information plays a central role in discussions of the nature of episodic memory (EM) (e.g., Tulving 1972; 1984; $2002 \mathrm{~b}$ ) and mental time travel (MTT) (target article). For this reason it is important to analyze the meaning of time in EM and MTT and to consider psychological research about memory for time and ways of thinking about the future. Among the different types of temporal information that humans and animals could process are: when an event occurred (or is expected to occur) within some time pattern ("temporal locations"), how long ago an event occurred ("temporal distances"), and before-after relations and other relations of the order of events (see Friedman 1993). Multiple representations and processes are involved in humans' memory for the times of past events and in their thinking about the times of future events (Friedman 2001; 2003). The impression we sometimes have that time is a seamless, linear continuum is at odds with the findings of research on temporal abilities and the processes that underlie them (Friedman 1993). For example, memory for time is often inaccurate, systematically distorted, and even inconsistent with remembering time in an integral way - we sometimes remember the time of day of an event but not the month or year.

Adults' sense of the times of past events depends mainly on inferring when the event must have happened by relating the content of the memory to one's general knowledge of personal, conventional, and natural time patterns. But adults, as well as children as young as 4 years, also have available impressions of the ages of events that provide limited information about their distances in the past (Friedman 1996; 2001). A differentiated sense of the future depends on mental representations of time patterns, probably supplemented from early childhood onward with propositions that are active in memory (e.g., that particular events are coming soon or won't happen for a long time) (Friedman 2003). This patchwork of processes, and the fact that humans remember and anticipate times separately on multiple time scales, reveals the complexity of memory for and anticipation of the times of events.

What temporal abilities are necessary to possess EM? At various stages in the development of his theory, Tulving referred to the following as critical features: temporally dated events (though not in conventional time units; Tulving 1972), coding the temporal relations among experienced events (Tulving
1972 ; 1984), and having a subjective sense of time (Tulving $2002 \mathrm{~b})$. Others have pointed to different time-related criteria (e.g., the ability to discriminate recent from remote events [de Kort et al. 2005] or the capacity to replay the flow of experience [Eichenbaumet al. 2005]), or they have maintained that temporal information is not necessary (Suddendorf \& Corballis [S\&C] in the target article; Zentall 2005). In light of research on memory for the times of events, S\&C's and Zentall's positions may be the wisest, at least for describing human memory. There is no evidence that events are automatically coded by the times of their occurrence or that memory is temporally organized (Friedman 1993; 2004); many older events are difficult to discriminate by their ages (e.g., Friedman \& Huttenlocher 1997) but are still presumably EMs; and it seems likely that we are poor at remembering the internal order of some EMs. It might be best to think of the relevant quality of EM as experiences that are remembered as occurring on a particular occasion.

In MTT, what does it mean to say that one is traveling through time? The metaphor can unintentionally imply a unity and continuity of time that is quite at odds with the fragmentary, manifold way humans experience it. The finding, mentioned earlier and cited by $\mathrm{S} \& \mathrm{C}$, that adults sometimes remember the time of day of an event but not its time on longer scales, is difficult to reconcile with the metaphor of "traveling through time." The limitations of this metaphor may be even clearer when one considers related developmental research. From about 4 years of age onward, many children are able to recall unique happenings when asked about events such as "your last birthday" or "last Christmas," but this ability appears years before children are aware of when these events had occurred relative to one another (Friedman 1992). What appear to be genuine EMs are more like "islands in time" than memories one reaches by mentally traveling through some temporally organized representation. Similarly, children anticipate particular events (and plan for them) before they have a clear understanding of when in the future the events will occur (Friedman 2003). Finally, 5-year-olds, who can remember specific past events and anticipate specific events to come, sometimes confuse the pastfuture status of these events (Friedman 2003).

Research on humans' memory for times and on thinking about the times of future events shows that there are some common processes (e.g., the use of representations of time patterns) and some differences (using impressions of the ages of memories). In my view, it remains an open question to what extent common processes underlie EM and future-directed thinking in general. The developmental and neurological evidence that $\mathrm{S} \& \mathrm{C}$ cite is suggestive, yet developmental changes can co-occur but be rooted in different processes, and the deficits that hippocampal patients show in EM and anticipation could be due in part to problems other than the capacity to engage in MTT (such as ones related to constructing spatially coherent representations; Hassabis et al. 2007). Even if temporal information is not a defining feature of $\mathrm{EM}$, as I and others have suggested, it is not clear that the remaining criterion - autonoetic consciousness of particular autobiographical episodes - is necessary to flexibly plan specific future events. Throughout development, planning may rely to a greater extent on information abstracted from repeated episodes (the commonalities of which are more relevant to the future than the particularities) and from semantic memory. S\&C have raised interesting questions which merit further research.

\section{Mental time travel sickness and a Bayesian remedy}

DOI: $10.1017 /$ S0140525X07002099

\section{Jay Hegdé}

Department of Psychology, University of Minnesota, Minneapolis, MN 55455.

hegde@umn.edu http://www.hegde.us 
Abstract: Mental time travel is a principled, but a narrow and computationally limiting, implementation of foresight. Future events can be predicted with sufficient specificity without having to have episodic memory of specific past events. Bayesian estimation theory provides a framework by which one can make predictions about specific future events by combining information about various generic patterns in the past experience.

Suddendorf \& Corballis argue persuasively that the ability to foresee future "situations" is likely to depend on many different mental faculties, including memory of the past. But despite recognizing the complexity of the prediction process, the authors focus on a surprisingly narrow and problematic mechanism for it, namely, mental time travel.

As the authors formulate it, mental time travel essentially treats future as a version of the past: What one is able to "prelive" about future events are those that one can relive about past events (target article, sect. 1, para. 1). The authors suggest that episodic memory helps "pre-live" future events, because it is this type memory that one needs for reliving the past. The key assumption here is that one can mentally create only those future events that one has specifically experienced in the past.

I contend that this is an unnecessarily narrow formulation of foresight, because one can obviously mentally create events that are sufficiently different from any that one has experienced before. The authors' formulation is also severely limiting because, if it were strictly true, it would mean that one would be able to foresee only those events that one has episodic memory of.

From the computational standpoint, it is clear that specific predictions about future events can, in principle, be made by using generic prior knowledge in a combinatorial fashion (see Glymour 2002). Information about the particularities of specific past events, such as that provided by episodic memory, is not needed. To cite a qualitative example, in order to foresee the possibility that I may be mugged if I walk through certain blocks of the city at night, I do not need the actual experience of having been mugged there at night. A general knowledge of risky time periods and risky neighborhoods is enough. This is because one can easily generalize and extrapolate, with arbitrary specificity and detail, from past experience. Thus, in the above example, one can not only foresee the possibility of being mugged, but also envisage the mugging event itself in arbitrary detail. Indeed, one can also vividly imagine events that one is certain never to have experienced in the past, such as a boulder rolling up a hill on its own. The point is that the authors' formulation of foresight ultimately amounts to placing patently untenable limitations on one's very ability to imagine.

Extending the authors' formulation of foresight to its logical limits, while perhaps not altogether fair to the authors, is nonetheless a useful exercise, because it reveals an instructive conundrum. To the extent that one can only foresee those future events that one has experienced in the past, and to the extent that events never repeat themselves exactly, one can never apply the memory of any past event to a future situation. Presumably, the authors would address this conundrum by allowing for some level of generalization and extrapolation, so that the future event does not have to be an exact replica of the past one. But that is precisely my point, too: Some degree of generalization and extrapolation is a prerequisite for predicting future events. But why limit it as severely and arbitrarily as the authors do?

The aforementioned logical exercise reveals another related, but more severe, computational limitation of the authors' formulation. Without the ability to extrapolate from generalities, the amount of particularities the brain would have to store would be subject to a combinatorial explosion. For every prediction of a future event, the memory of a corresponding past event would be needed. Conversely, what one can predict about the future will be limited by one's episodic memory. In the aforementioned mugging example, in order to foresee a mugging event, I would have to have the memory of having been previously mugged by the same person, and in the same city block, and so forth.

Again, the authors would presumably address this handicap by allowing some generalization across, and extrapolation from, past experiences. Doing so would, among other things, recognize that the various types of memory are not quite as distinct, and independent, from each other as one might think. That is, different forms of memory might interact with each other and with other mental faculties to help foresee the future. Although the authors allude to this possibility initially, they move away from it later, especially in rejecting several possible instances of foresight in nonhuman animals simply because they do not appear to involve episodic memory (target article, sect. 3).

Note that in terms of its amenability to generalization and extrapolation, episodic memory is the least suitable form of memory. That is, episodic memory by itself is a computational bottleneck. Therefore, other types of memory must play a major role, and mental time travel must play a correspondingly smaller role, in foresight.

The Bayesian estimation theory encapsulates the aforementioned general computational principles into a powerful and flexible framework for making predictions. Briefly, in this framework, prediction is a fairly straightforward extension of parameter estimation. The future value of a given parameter can be estimated by combining the relevant probabilistic information about the past and present values of the parameter (for more rigorous expositions, see Davidson \& Wolpert 2005; Glymour 2002; Krauth 1983). Three features of the Bayesian framework are especially worth highlighting in this context. First, this framework is clearly biologically plausible. Second, in many cases, Bayesian prediction can be shown to be ideal. Third, the Bayesian framework is versatile, in that it can use all available relevant information, including different forms of memory, to arrive at a prediction. Thus, the Bayesian framework can utilize episodic memory, but is not dependent on it. In this sense, the Bayesian framework subsumes, and greatly extends, the authors' framework for foresight.

Of course, the Bayesian framework for prediction has its faults and limitations (see, e.g., Krauth 1983). But it represents, at a minimum, a substantive counterexample to the framework suggested by the authors.

\section{ACKNOWLEDGMENT}

Preparation of this commentary was supported by ONR grant N00014-05-1-0124 to Dr. Daniel Kersten.

\section{Past and future, human and nonhuman, semantic/procedural and episodic}

DOI: 10.1017/S0140525X07002105

\author{
James R. Hurford, Molly Flaherty, and Giorgis Argyropoulos \\ Language Evolution and Computation Research Unit, School of Philosophy, \\ Psychology and Language Sciences, University of Edinburgh, Edinburgh, EH8 \\ 9LL, United Kingdom. \\ jim@ling.ed.ac.uk http://www.ling.ed.ac.uk \\ s0672076@ling.ed.ac.uk s0677134@ling.ed.ac.uk
}

Abstract: The overlap of representations of past and future is not a completely new idea. Suddendorf \& Corballis (S\&C) usefully discuss the problems of testing the existence of such representations. Our taxonomy of memory differs from theirs, emphasizing the late evolutionary emergence of notions of time in memory.

The target article makes a useful contribution. We offer some reservations that do not undermine its central purpose. 
Suddendorf \& Corballis (S\&C) join company with the literature from the past three decades casting doubt on a radical psychological past/future (memory/planning) distinction. Whereas memory was once thought of as exclusively retrospective, it is now recognized that "prospective memory" (a term introduced by Meacham \& Singer 1977) shares features with retrospective memory. Cook et al. (1983) also demonstrated the overlap between retrospective memory and prospective memory in rats. Tulving (2005) identifies episodic memory closely with both past and future (i.e., planned) events.

We are not so convinced that nonhumans lack any retrospective episodic memory. Sue Savage-Rumbaugh (personal communication) tells of promising Kanzi, the well-known bonobo, a treat tomorrow and then being reminded of the promise the next day. Kanzi's specific desire may not have waned overnight, so there was not necessarily any representation of a future mental state distinct from the present, but Kanzi did recall the specific event of being promised. For retrospective cases, the question is whether, when an animal observes something happening, it stores a stripped-down "episodic" memory of the particular event, or makes some inferences about the lasting state of the world, such as "food behind tree." King, the gorilla tested by Schwartz et al. (2004), was shown events with no lasting consequence on the world, such as a man skipping. King could show that he recalled these events, as compared with distractor suggestions of other events which he might have (but had not) seen. But King was only tested 15 minutes after observing the event. The data are sparse, and we agree with $\mathrm{S} \& \mathrm{C}$ that there is a large quantitative gulf between humans and nonhumans for retrospective episodic memory.

Designing experiments to test the distinction concerned is challenging. We suggest a sense in which considerations of parsimony can conceivably be applied. Obviously no animal, human or otherwise, stores all the information from an observed event. If an animal remembers a specific episode, how much selection of its details happens? If, on the other hand, the animal does not store the episodic information, but only certain inferences relevant to its own typical needs, how many such inferences does it make? S\&C's itemization of the components of "www" is useful. Hurley (2006) describes humans as "inferentially promiscuous." Does there come a point when we have to list so many different types of "w" inferred by an animal from an event that it becomes more parsimonious to assume that the animal just stored a stripped-down episodic memory of the event itself?

We agree with S\&C that Mulcahy and Call (2006) come close to demonstrating planning by animals for anticipated needs. Mulcahy and Call (2006) showed that some bonobos in their experiment collected a specific tool for use on a specific foodretrieving task as much as 14 hours later. S\&C's scrupulous critique of this experiment is correct, and there indeed remains the possibility that the animals just got into the habit of selecting the right tool. A very similar procedure to S\&C's suggested red and green light future-planning food caching experiment has in fact been successfully completed by scrub jays (Raby et al. 2007), but S\&C would judge that this is also not sufficient, because of the limited scope and inflexibility of the behavior. If these objections are valid, then radically different criteria for thinking about mental time travel (MTT), far from any sort of www components, are in order. We commend S\&C for setting out just such criteria.

S\&C's theater metaphor is a bold departure, and it provides fruitful ground for new investigation. That said, several components show evidence of insufficient development. Most particularly, it is not clear that the "broadcaster" component is integral to their framework. Does episodic memory only become episodic when it is shared with others? It seems unlikely that S\&C would wish to make this claim, but by including the broadcaster as one of their seven components, the reader is left to think that episodic memory requires verbal sharing. This precludes possible research on episodic, or episodic-like, memory in nonhumans.

Although it plays no central role in S\&C's main discussion of MTT, we take issue with their taxonomy of memory and prospection systems in their Figure 1. We see the picture as radically different. We would bracket procedural and semantic memory together as timeless forms of memory, evolutionarily preceding the emergence of episodic memory, in both its retrospective and prospective forms. Indeed, when S\&C appear to distinguish between "semantic memory and prospection," we find this hard to interpret. Semantic memory encodes tenseless facts like "sugar be sweet." Likewise, procedural memory encodes instructions on what action to take whenever certain circumstances arise. Both procedural and semantic memories are laid down by experience, which is necessarily temporally prior to their formation, but they are not memories about particular events in the past, still less about events in the future. So we agree with S\&C's statement that "The mental reconstruction of past events and construction of future ones may have been responsible for the concept of time itself, and the understanding of a continuity between past and future" (sect. 2.1, para. 3). This entails that the emergence of past/future episodic memory was also the emergence, for the first time in evolution, of types of mental representation incorporating notions of past and future time. This is, of course, a different matter from semantic or procedural memories encoding facts about temporal order, for example, that thunder follows lightning.

There is now increasing evidence for coinvolvement of motor and sensory components in memory for objects and actions, tending to conflate the semantic/procedural distinction. Hommel et al. (2001) give an overview pointing to the conclusion that "Perceived events (perceptions) and to-be-produced events (actions) are equally represented by integrated, task-tuned networks of feature codes - cognitive structures we call event codes" (p. 849). Hurford (2007) gives a short survey of other evidence; see also Martin et al. (1996), who found that "naming tools selectively activated a left premotor area also activated by imagined hand movements." Similarly, Mecklinger et al. (2002) concluded that "visual working memory for manipulable objects is based on motor programmes associated with their use" (p. 1115). Gibson's (1979) idea of affordances depends crucially on perception-action coupling. The discovery of mirror neurons also shows overlaps between sensory and motor representations of actions. In philosophy, a school of thought known as "Enactive Perception" also emphasizes the involvement of action in perception; see Noë (2004).

Finally and quite speculatively, we mention a kind of mental representation which, though essentially semantic/procedural, encodes information about typical types of complex events brought to mind by humans in either a retrospective or a prospective sense. This is the idea of "scripts," such as the complex structure of things to do when going to a restaurant (Schank \& Abelson 1977). Such scripts form a framework for our retrospective and prospective representations of events. The ability to conjure up such scripts is a kind of general mental space integration capacity.

\section{Memory, imagination, and the asymmetry between past and future}

DOI: $10.1017 / \mathrm{S} 0140525 \mathrm{X} 07002117$

\section{Bjorn Merker \\ Gamla Kyrkvagen 44, SE-14171 Segeltorp, Sweden. \\ gyr694c@tninet.se}

Abstract: A number of difficulties encumber the Suddendorf \& Corballis $(\mathrm{S} \& \mathrm{C})$ proposal regarding mental time travel into the future. Among these are conceptual issues turning on the inherent asymmetry of time 
and causality with regard to past and future, and the bearing of such asymmetry on the uses and utility of retrospective versus prospective mental time travel, on which I comment.

Suddendorf \& Corballis (S\&C) propose that the capacity to mentally transport oneself into future situations imagined in concreto is a uniquely human cognitive adaptation that has not only played a decisive role in our evolutionary past but may also account for a large part of our current dominance on Earth. The case they make for this is, however, encumbered with difficulties, of which I will comment on a few largely conceptual matters.

Foresight - the ability to anticipate future needs and to act accordingly - is the key concept and source of utility in the authors' account. As they are well aware, foresight can be served by either direct semantic prospection or more round about mental time travel directed to the future. In fact, every single example illustrating foresight by means of mental time travel provided in the target article can be implemented by direct semantic prospection instead. But that means that those examples illustrate only the utility of foresight - which no one doubts - but not the specific utility of mental time travel for that purpose, as intended and implied by the authors. Thus, rehearsal for questions that may be posed in a forthcoming job interview (sect. 2.1) is readily accomplished through semantic prospection ("I wonder what questions I'll get? Maybe this one..." etc.). What is more, S\&C provide no concrete evidence that mental time travel ever yields information whose efficacy for success in planning for the future exceeds that provided by semantic prospection. Such information may in fact be unavailable in principle, for reasons connected with the nature of time and its relation to memory and imagination.

Occasional qualifications made in passing notwithstanding, the authors' entire argument is built upon a perfect symmetry between past and future, as strikingly illustrated in Figure 1 of the target article. Yet time itself, along with the causality of the macroscopic world, is profoundly and fundamentally asymmetric with respect to past and future. The past has actually happened, which means that it once was the present, and thus subject to memory storage by suitably equipped organisms (which is how it became "the past"). That means that in principle, at least, the possibility of veridical memory exists. There is no corresponding possibility with regard to the future, because the future has by definition not happened, being a mixture of coexisting latent possibilities as yet unresolved. Which of these is the "true future" cannot be known until it has "travelled to us," and become the present. Similarly for causality: In the macroscopic world the effect follows the cause in time, but never the reverse. That is how the present (cause) becomes a memory (effect) by the next present along future-directed causal pathways.

It is the existence of a more or less veridical memory for the past which, on occasion, lends utility to revisiting that past in the imagination through mental time travel instead of relying on the distillate of that past provided by semantic memory. Let us say new circumstances have rendered a detail that did not seem important at the time relevant to our present concerns. Occasionally, we are in fact able to recover such detail by going back and "reliving," as it were, the situation in question, though that utility is in all likelihood a rather marginal one. The veridical memory is the "destination" towards which we steer in retrospective mental time travel. There is no such destination for prospective mental time travel, because unlike the past, the future has not happened and all we can know is that all possible futures, except one, will in fact not materialize, but not which one.

That is, the great flexibility of future time travel which the authors tirelessly extol as its great advantage is to no avail as far as foresight is concerned, because the utility of anticipating the future for prudential purposes does not hinge on the number of imagined alternatives, but on being correct, and such prediction is possible only to the extent that the future is in fact foreseeable, which means being "like the present and the past" (see next paragraph). Moreover, nothing is less certain in that regard than the fine grain ("particularities") of imagined futures, the one additional advantage ascribed by $\mathrm{S} \& \mathrm{C}$ to actual mental time travel compared to semantic prospection. These points can be illustrated by the importance of correctly anticipating which questions will in fact be asked in a forthcoming job interview. Preparation for the wrong questions is wasted effort, and may even act as an impediment during the interview. Needless to say, mental time travel possesses no privileged power to pick the right questions.

What are we to make, then, of the striking parallels the authors array between past and future in human performance? In light of what has gone before, the answer is readily available. The only aspects of the future that are in fact predictable are those respects in which it continues to be like the past (at all time scales and in any number of attributes and statistical characteristics). When, therefore, we construe possible futures, they share vast domains of content with the present and its past states of variation vouchsafed by memory, whether semantic or episodic. In doing so, we are in fact in large measure projecting the past into the future, abstractly or concretely, and not "travelling" into it. The parallels listed by $\mathrm{S} \& \mathrm{C}$ follow as a matter of course.

To summarize: What the authors call mental time travel into the future is prospective fantasy and the use of imagery in scenariobuilding (for the latter in relation to the frontal lobes, see Nauta 1971). These have their uses, in various creative endeavors, say, endeavors which certainly may affect and change the future. That, however, is a matter of the extent to which those endeavors recruit workable causal channels for their implementation, and not of any special efficacy for actually anticipating the future on the part of the fantasies that inspire them, as the record of failed prospective fantasy supplied by human history reminds us.

\section{Has mental time travel really affected human culture?}

\author{
DOI: $10.1017 /$ S0140525X07002129
}

\author{
Alex Mesoudi \\ Department of Social and Developmental Psychology, University of \\ Cambridge, Cambridge CB2 3RQ, United Kingdom. \\ am786@cam.ac.uk http://amesoudi.googiepages.com
}

\begin{abstract}
Suddendorf \& Corballis $(\mathrm{S} \& \mathrm{C})$ claim that mental time travel has significantly affected human cultural change. This echoes a common criticism of theories of Darwinian cultural evolution: that, whereas evolution is blind, culture is directed by people who can foresee and plan for future events. Here I argue that such a claim is premature, and more rigorous tests of S\&C's claim are needed.
\end{abstract}

In the final section of their fascinating target article, Suddendorf \& Corballis $(\mathrm{S} \& \mathrm{C})$ propose that mental time travel has important implications for human culture: "Law, education, religion, and many other fundamental aspects of human culture are deeply dependant on our shared ability to reconstruct past and imagine future events" (sect. 6, para. 3). Yet, besides some informal speculation regarding stone tools and the use of fire (which, as the authors acknowledge, are "just-so stories"), the specific implications of mental time travel for human culture, and for research in the social sciences concerning cultural change, are left unexplored. It is important to distinguish between the capacity for culture and the contents of culture (Mesoudi et al. 2006). While mental time travel potentially has implications for both, I focus here on the latter, that is, the effect of mental time travel on changes in the contents of human culture, such as law, education, and religion.

The existence of mental time travel, specifically regarding future events (i.e., foresight), has direct relevance to an ongoing debate concerning the validity of the theory of Darwinian cultural 
evolution. Recent years have seen a burgeoning interest in cultural evolution, the idea that human culture changes according to the same fundamental principles as do biological species, and that consequently many of the same tools, methods, theories, and concepts developed by evolutionary biologists can be adapted for use by cultural scientists to analyze cultural change (Aunger 2000; Henrich \& McElreath 2003; Mesoudi et al. 2004; 2006; Richerson \& Boyd 2005). Despite this growing movement, cultural evolution is still met with much hostility and opposition from many quarters, especially the social sciences most directly involved in the study of human culture, such as cultural anthropology and sociology. One of the most oft-cited criticisms of evolutionary approaches to human culture is that, whereas biological (gene-based) evolution is "blind" and undirected (Dawkins 1996), culture is directed by conscious and intentional human agents who can use their capacity for foresight to guide cultural change towards specific goals (e.g., Benton 2000; Bryant 2004; Carneiro 1985; Chater 2005; Dasgupta 2004; Hallpike 1986). For example, Benton (2000) criticizes Darwinian models of cultural change because "human agents act intentionally to produce anticipated outcomes: They are not 'blind watchmakers"” (p. 216).

The initial reaction from advocates of Darwinian cultural evolution to such criticism was to deny that humans possessed foresight, and to argue that cultural change is just as blind as biological change (e.g., Campbell 1960; Rindos 1985; Simonton 1999). The existence of mental time travel, which, as S\&C show, is supported by a rich body of evidence from neuropsychology and developmental, cognitive, and comparative psychology, appears to make this position untenable. Mental time travel allows people to simulate potential future scenarios in order to anticipate and plan for novel future events in a way that does not appear to have any parallel in biological evolution. Biologically evolved biases in learning can "predict" the future based on past regularities (Lorenz 1969; Mayr 1982), but biological evolution cannot actively simulate novel future events.

So does the existence of mental time travel invalidate the theory of Darwinian cultural evolution? Although it is possible, such a conclusion would be premature. First, it has yet to be established empirically that mental time travel has affected cultural change in a significant manner. This must be addressed not with just-so stories or vague talk of "intentional actors," but by integrating the body of work from the behavioral and psychological sciences reviewed by $\mathrm{S} \& \mathrm{C}$ with evidence from the social sciences regarding actual cultural change. Existing studies (e.g., Basalla 1988) suggest that foresight plays little role in directing technological change, although such studies are relatively informal and do not make the important theoretical distinctions that follow from the work reviewed by S\&C, such as between "semantic foresight" (i.e., script-like expectancies generated by semantic memory) and "episodic foresight" (i.e., forward-looking mental time travel). Future studies might simulate cultural change in computer-generated agents (Epstein \& Axtell 1996) who possess varying degrees of foresight, from "no foresight" to "semantic foresight" to "episodic foresight" to "omniscience," along a "continuum of mindfulness" (Dennett \& McKay 2006), and match the resulting cultural dynamics to actual historical, archaeological and sociological data. Lab-based experimental simulations of cultural transmission and cultural evolution (Mesoudi 2007) might test the extent to which the episodic memory system is used to maintain complex cultural traditions, while ethnographers might quantify the long-term accuracy and consequences of episodic foresight in traditional societies.

Second, even if it was established that mental time travel has significantly affected cultural change, this does not automatically invalidate Darwinian approaches to culture. Even though people can simulate future scenarios, there is no guarantee that this simulation will be accurate. As S\&C note, "Mental time travel is of course not to be mistaken for clairvoyance" (sect. 4.5). The well-documented inaccuracies in episodic memory (Loftus
1996; Loftus \& Ketcham 1994) suggest that episodic foresight is similarly biased and inaccurate. Indeed, some of these biases have already been identified, such as the planning fallacy (Kahneman \& Tversky 1979) or hyperbolic discounting (Kirby 1997). Critics of cultural evolution commonly conflate this imperfect "human foresight" with the perfect "supernatural foresight" of an omniscient being. Biology is blind in the latter sense, but so is culture: There is no omniscient being with perfectly accurate supernatural foresight guiding cultural change. Unfortunately, most critics of cultural evolution do not make this distinction, and assume that any kind of foresight automatically invalidates Darwinian cultural evolution. Cultural evolution does not have to be identical in every respect to biological evolution, and evolutionary models of culture have already successfully incorporated phenomena not found in biological evolution, such as blending inheritance (Boyd \& Richerson 1985); perhaps the same can be done with mental time travel.

In summary, researchers who study culture would benefit from explicitly incorporating the theoretical distinctions identified by S\&C into their work, while S\&C's proposal would benefit from a more detailed consideration of evidence from the social sciences regarding actual cultural change.

\section{Developing past and future selves for time travel narratives}

\section{DOI: $10.1017 /$ S0140525X07002130}

\section{Katherine Nelson \\ Department of Psychology, City University of New York Graduate Center, New York, NY 10016-4309. \\ knelson@gc.cuny.edu}

Abstract: Mental time travel requires the sense of a past and future self, which is lacking in the early years of life. Research on the development of autobiographical memory and development of self sheds light on the difference between memory in other animals and its cultural narrative basis in humans.

Suddendorf \& Corballis's (S\&C's) claim that memory systems are adaptive for their contribution to future survival is consistent with Tulving's $(1983 ;$ 2005) arguments, and with my proposal for the evolution and development of memory (Nelson 1993a; 1993b; 2005). In considering the emergence of autobiographical memory in childhood, Nelson and Fivush (2004) proposed a constellation of contributions to this manifestation of time travel, similar to S\&C's proposal of a constellation of mechanisms responsible for foresight. We emphasized the development of representational language, conversational exchanges about past and future, and cultural practices, thereby placing more weight on the codevelopment of culture and biology in the emergence of episodic memory - and foresight - in both phylogeny and ontogeny than S\&C do. The neglect of culture in mind in S\&C's account is in my view a serious drawback to their account of the uniqueness of the human ability to remember the past and foresee the future.

Prominent among the achievements Nelson and Fivush (2004) identified as necessary to autobiographical memory was skill in narrative construction and understanding and its use in personal memory recounts. Narrative is a unique cultural production, as universal in human societies as language itself. It provides the structural glue that ties together the who, what, where, when, and why that S\&C recognize as necessary to complex foresight. But their theater metaphor strangely neglects the essential structure of narrative, the plot or drama that their "playwright" must produce. Instead, they imagine the playwright picking and choosing among pieces of prior specific episodic memories to make up a new scenario. But without the structure of a narrative, situated in a specific cultural setting, the play - the memory or 
foretelling - is untethered. This is well observed in the many extensive records of children's contributions to memory talk now in the literature, which begin with bits and pieces of scenes, and only with practice and the help of older conversational partners eventually come to resemble narratives of a "self" experience with a beginning, identified characters, setting, some highpoint, and ending, perhaps with an evaluation. Autobiographical memory consisting of narratives from the personal past emerges gradually over the preschool years as children gain practice in reminiscing with others about things they have experienced together or separately (Nelson \& Fivush 2004). There is much less research on children's experience in talking about the future, but there is evidence that future talk is less frequent than talk about the past and occurs more with older preschoolers (Eisenberg 2006), suggesting that personal memory is the key to the child's engagement in time travel.

From the perspective of these developments it seems clear that, as Tulving claimed, the unique time travel addition in human evolution is not in foreseeing the future, but rather, the ability to recall the self in specific past events, that is, episodic memory (EM). EM may serve the future, as does all memory, but this is not its unique function. General scripts may be more useful for predicting what will happen next, allowing for flexibility through open slots and different paths. In fact, very young children tend to focus on the general "way things are" rather than on the particularities of a specific past. This makes functional sense when the goal is to understand, anticipate, and participate in the social world. Declarative memory (facts about the world, including routines and scripts) is a collection of notnow know-how and know-about, and may also include episodic fragments and even singular episodes, without specifying that they "happened to me at a particular time in the past." In contrast, EM does preserve specific complex events of personal significance. EM is the only form of memory that is about the past; it is also the only form that is about the self (autonoetic) (Tulving 1993).

Self-memory is late in developing, and is followed by developing ideas of a self-future. The critical questions about these emerging senses of self in time - in development as in evolution - are why and how? Children begin reporting on specific past episodes (around 3 years of age), but it is several years before most children fluently compose narrative accounts of their own experience, or specific plans for the future. Traditional developmental accounts assume that egocentrism is characteristic of early childhood. Therefore, the slow development of understanding of self in time is not expected, and relatively little research has been devoted to it (Moore \& Lemmon 2001; Nelson 1996; 2001).

Early in development self-experience is the source of all memory, because one person's experience is only accessible to another through language, which infants and young children are not privy to. Thus, the only experiencer in a child's early memory is the $I$. It is not until the child has acquired sufficient skill with language to engage in conversations with others about their past and future experiences that identifying different experiencers other than the self becomes critical. Hence, only when the child is exposed (through language) to contrasting pasts and others' futures that the child's own self-in-the-past becomes salient, distinguished from a generalized "not now" held in common with others.

Relatedly, younger children remain ignorant about or indifferent to the source of their memory - or of information in general - up to the age of about 6 years. Younger children typically do not monitor whose memory is reported or where a particular bit of knowledge arrived from (Roberts \& Blades 2000). This is understandable, given that the basic memory system conserves information from the child's (or more generally, an animal's) own experience in the social and physical world. It is only humans who share information about happenings that they have individual knowledge of. Therfore, only humans must focus attention on sources, specifically between self- experience or others' report. Then the self-narratives of autobiographical memory emerge from a murky past in culturally supported forms learned through everyday social interactions during the preschool years. These complexities of the developing self in the social world strongly imply that the emergence of both personal memory and personal possible futures is attributable to the social and cultural conditions and narrative framing of human lives and experiences.

\section{Prospection or projection: Neurobiological basis of stimulus-independent mental traveling}

\author{
DOI: $10.1017 / \mathrm{S} 0140525 \mathrm{X} 07002142$
}

\section{Jiro Okuda}

Tamagawa University Brain Science Institute, Machida, Tokyo 194-8610, Japan; and Institute of Cognitive Neuroscience and Psychology Department, University College London, London WC1 N $3 A R$, United Kingdom.

\section{j.okuda@lab.tamagawa.ac.jp}

http://www.icn.ucl.ac.uk/executive_functions/People/Jiro.html

\begin{abstract}
The number of studies concerning the neurobiology of human prospection is now rapidly exploding. Recent works suggest that prospection can be better understood in a broader context of selfprojection into other times, places, or agents that can share the same cerebral basis involving medial aspects of prefrontal, parietal, and temporal cortices. Mental time travel may be extended more generally to "mental traveling," accomplished by stimulus-independent mental processes typical of human thought.
\end{abstract}

When we make a future plan or intend to organize future behavior, we consciously or unconsciously remember our past experiences or acquired knowledge and utilize them as an effective guideline to construct ideas about the future... In other words, we are unable to have a good insight into the future without reactivating past experiences or general knowledge. (Okuda et al. 2003)

In the target article, Suddendorf \& Corballis (S\&C) formally propose a framework for linking cognitive/behavioral systems concerning memory (capacity for past experiences) with prospection (future-oriented behavior/cognition). Their primary argument is that the system, both for memory and prospection, is hierarchically organized according to levels of flexibility and singularity, and that the most flexible, "episodic" level heavily depends on the uniquely human ability of "mental time travel." The argument is motivated largely by recent comparative studies of animal behavior and developmental studies in humans. Although these behavioral observations are amazing and extremely valuable for considering memory-prospection systems in humans and animals, a growing body of neurobiological studies also provides a seminal contribution to the theory. The target article refers to some of the most essential elements of such an approach, but seems to lack enough documentation on what the neurobiological data can suggest to the human ability of foresight. This approach has only recently begun to draw researchers' attention, but a rapid expansion of both empirical data and theoretical concepts has taken place recently. In the following, I make some comments on the very recent advances in the neurobiology of human prospection.

S\&C briefly introduce evolutionary change in the relative size of certain areas of the human brain, especially the prefrontal cortex, as a neurobiological index of differing capacity for mental time travel in humans and other animals. However, a more direct and informative way to understand how mental time travel functions in the brain is perhaps to seek distinguishable brain activity while participants are engaged in tasks requiring mental time travel. S\&C mention potential involvement of cortico-basal ganglia loops in anticipation of future rewards evidenced by brain imaging studies employing tasks in which 
participants made a decision through learning of a stimulusaction-reward contingency. This series of processes involved with reward-based decision making is obviously one form of prospection, but, as was defined in the target article, it is best described as "procedural future cognition" that might be less flexible and stimulus-bound. That is, we cannot be sure whether the participants vividly imagine a particular future event of "gaining reward" in its unique detail upon deciding an action, when repeatedly presented with prototypical experimental stimuli such as geometrical shapes.

Probably a more direct approach to uncover the cerebral bases of mental time travel is brain imaging during stimulus-free, episodic recall. Okuda et al. (2003) performed a simple but challenging experiment investigating brain activations while participants orally described particular events of certain time periods in the future and the past. They found that a basically similar brain network involving medial aspects of the prefrontal, temporal, and parietal cortices was activated during both thinking about the future and about past events, and that magnitude of the activity in each subregion was characteristically modulated by future/past orientation and temporal distance from the present.

Stimulated by this demonstration, several follow-up studies have recently been pursued. Addis et al. (2007) controlled more strictly for phenomenological quality of remembered/imagined events so that they were truly "episodic" and that details, emotionality, personal significance, and field/observer perspective were constant across the future and past conditions. They utilized a technique of event-related functional magnetic resonance imaging (fMRI) to dissociate brain activity patterns during event construction (search and reconstruction of autobiographical event information) and elaboration (subsequent retrieval/ imagination of supplementary details). Under such a wellcontrolled experimental setting, the common activation in the core network of the medial prefrontal-temporal-parietal cortices were observed particularly during the event elaboration, which might be considered a consequence of greater demand of the mental time travel during the event elaboration both for the future and the past. Szpunar et al. (2007) also used eventrelated $\mathrm{fMRI}$ and confirmed common engagement of this core network in remembering/envisioning of self-relevant, autobiographical events in the past and future. Apart from the functional brain imaging, Hassabis et al. (2007) have demonstrated striking evidence that amnesic patients with bilateral medial temporal damage cannot imagine new experiences in addition to their impairment in remembering past episodes. Importantly, the deficit was not restricted to personally relevant possible future events (e.g., a possible Christmas event) but was common to wide variety of commonplace scenarios (situation of standing in the main hall of a museum, etc.), with severe impairment in placing fragmented images into one coherent spatial context.

These sets of novel evidence and other related data have led Buckner and Carroll (2006) to propose that envisioning the future (prospection), remembering the past (episodic memory), conceiving the viewpoint of others (theory of mind), as well as shifting one's topographical perspective (some forms of spatial navigation) reflect the workings of the same core brain network, that is, the medial prefrontal-parietal-temporal network. Therefore, although the claim by S\&C that evolutionary significance of episodic memory primarily lies in its adaptive advantage for future survival through flexible mental time travel can still be true, available neurobiological data appear to suggest that the mechanism should be extended to a broader context of "mental traveling," not only in the time domain but also in the spatial context and others' minds.

What, then, is the fundamental mechanism by which theses various domains of mental traveling work with the same core brain network? Buckner and Carroll (2007) assume "selfprojection" as a basic facility. Burgess et al. (2005; 2006) propose a more explanatory model, although it only considers, at the least explicitly, a mechanism involving vicinity of the frontal part of the core regions. The model, based on careful reviews of both neuropsychological and functional imaging data, proposes that the anterior part of the prefrontal cortex acts as a gateway that modulates an attentional bias between stimulus-independent mental processes and stimulus-oriented immediate cognitions. It is plausible that cognitive operations, independent of environmental stimulus and motor responses to it, are the most fundamental requirement for mental travel to any other objectives that are not present in one's immediate perceptual-motor representations. This general idea is also confirmed by other brain imaging studies indicating roles of the anterior part of the prefrontal cortex in prospective memory or remembering mentally represented action plans during ongoing cognitive activities (e.g., Okuda et al. 2007), as well as involvement of medial prefrontal and parietal cortices in stimulus-independent thought or mind-wondering (Mason et al. 2007a, but see also Gilbert et al. 2007 and Mason et al. 2007b). Moreover, the argument of stimulus-independent and stimulus-oriented cognition is directly relevant to the distinction between "declarative" and "non-declarative" future prospection as discussed in the target article. Probably there is a corresponding neurobiological distinction between the two in the brain: The stimulus-oriented, non-declarative prospection may depend largely on cortico-basal ganglia loop, whereas the stimulus-independent, declarative prospection may recruit the medial prefrontal-parietal-temporal network. Then how can the two prospection systems coexist or switch one another? Again, the gateway model explains regulation between the two systems as a primary function of the anterior portion of the prefrontal cortex.

One final comment on the relation between the theater metaphor and the neurobiologically plausible mental traveling hypothesis I have raised earlier: How does the assumption of stimulus-independent mental traveling to other times, places, and individuals accommodate with the theater metaphor? It seems to me that the set, stage, and actor components roughly correspond to the time, place, and agent domains, respectively. Again, regulation between stimulus-oriented immediate cognition and stimulus-independent mental processes, possibly a role played by the executive producer, may be a critical process for well-organized mental traveling to function.

\section{ACKNOWLEDGMENTS}

Preparation of this manuscript was partly supported by 21st Century Centre of Excellence (COE) program for Tamagawa University from Japan Society for the Promotion of Science (JSPS) and by Grants-in-Aid for Scientific Research for Jiro Okuda (\#19650064 and 19330161) from the Ministry of Education, Culture, Sports, Science and Technology (MEXT).

\section{What are the evolutionary causes of mental time travel?}

\section{DOI: $10.1017 / \mathrm{S} 0140525 X 07002154$}

\section{Mathias Osvath and Peter Gärdenfors \\ Cognitive Science Department, Lund University, Lund, SE-222 22, Sweden. mathias.osvath@lucs.lu.se \\ http://www.fil.lu.se/lucs/staff/person.asp?id=155\&lang=eng peter.gardenfors@lucs.lu.se \\ http://www.fil.lu.se/lucs/staff/person.asp?id $=42$}

\begin{abstract}
We are not entirely satisfied with the evolutionary explanation provided by Suddendorf \& Corballis (S\&C) for why only humans should be capable of advanced mental time travel. General social factors do not suffice, given that other primates are also highly social. We discuss the evolutionary mechanisms that have generated mental time travel typical to humans, focusing on ecological factors.
\end{abstract}

First of all we applaud Suddendorf \& Corballis $(\mathrm{S} \& \mathrm{C})$ in their efforts to write a much needed, comprehensive review on 
mental time travel (MTT). We agree with S\&C that MTT is a complex, multi-component skill with several advanced cognitive mechanisms working in concert. However, we do not reach the same conclusion when considering the evidence for MTT in nonhumans. S\&C argue that the lack of evidence for MTT in animals makes it reasonable that it is unique to humans. We think that one should be agnostic about the human uniqueness claim. The study of mental time travel in nonhumans is so far extremely limited, and actually indicates mostly positive results (e.g., Dally et al. 2006b; Mulcahy \& Call 2006; Naqshbandi \& Roberts 2006; Raby et al. 2007). Undoubtedly MTT plays a decisive part in human life and society, at the same time as it is extremely hard to observe in nonhumans. MTT is certainly typical of humans, but in what way? It may be that humans are the most apt mental time travelers when it comes to the technical or physical aspects of the world, but that we might not be lonely travelers in the social domain.

In section 6 of the target article, $\mathrm{S} \& \mathrm{C}$ focus on social factors when discussing evolutionary pressures. A problem with this proposal is that it does not explain why advanced MTT should not exist among other apes or animals. Social interaction within many species is very strong. So, if sociality is the crucial factor behind the evolution of mental time travel, it should be expected in some other primates. In fact, we would not be surprised if some primates are proficient mental time travelers within the social domain. The problem is a methodological one: It would be very hard to isolate behaviors connected to MTT when observing the social life of nonverbal creatures; such behaviors could be explained in a leaner way than assuming MTT. If we were to observe humans, or hominins, without attention to language, we would rely on the behaviors surrounding artifacts to identify MTT. De Waal (1982) noted that the advanced social cognition apparent in great apes probably was a stepping-stone to the complex artifactual cognition in humans. He observes that humans and apes seem almost equally developed in social cognition, though differing widely in technical understanding.

The hand-to-mouth lifestyle of great apes does not apply to humans. In order to understand the typicality and the evolution of human MTT, one must also pay attention to the differences in ecology. Following Osvath and Gärdenfors (2005), we argue that the Oldowan culture, 2.6 to 1.5 million years ago, constituted an ecological niche that enhanced the ability to mentally represent a possible future. MTT might to some extent already have been in place as a result of earlier selection pressures in the social life, but changes in hominin ecology chiseled out the more advanced ability that we seem totally dependent upon nowadays. The main components of the Oldowan culture are recognized as: (1) the manufacturing and use of stone tools; (2) the transport of artifacts (at least stone tools); (3) the transport of pieces of carcasses; and (4) the use of accumulation spots (Plummer 2004). A significant advantage of this culture is that it enabled a much wider exploitation of meat resources.

Savannah conditions offered some hominins a wider variety of food sources, and these food sources were more transient and scattered than those exploited by other primates. Therefore, the day ranges of the early hominids were more extended than those of extant apes (cf. Bickerton 2002). The Oldowan life style was signified by an extension in time and space. The fitness of the hominids in this niche increased with adaptations for long ranging, as indicated by the skeletal remains. These morphological adaptations must have been related to behavioral adaptations, which could be a result of an evolving mental time travel.

One example, supporting this general argument, concerns the curated technology of the Oldowan culture. As S\&C mention, there is clear evidence that transport of the artifacts (at least the stone tools) was an important trait of the culture (Toth 1985). It is not possible to know exactly where the next fresh kill will be found; it might be several kilometers away from nearest raw material source. Without sharp-edged stone tools in the immediate vicinity, a carcass would lose much of its value. Another aspect of Oldowan culture seems to be the saving of a tool (or a core) after it has been used.

A second example concerns the division of labor. This factor could in fact be used to turn the group of hominids into a virtual Swiss army knife. Some individuals might carry throwing stones, some might carry children, and others could carry water or wooden tools; some may go scavenging or hunting while others may focus on gathering food. Division of labor in the modern human form of hunting and gathering is deeply dependent on mental time travel. The individual must in some sense be able to imagine other individuals currently outside his or her immediate sensory scope doing their part of the job.

These examples present some reasons why complex mental time travel evolved to the human level within the Oldowan culture - or earlier (for a more detailed account, see Osvath \& Gärdenfors 2005). Once the period of Acheulean tools was reached, beginning about 1.5 million years ago, mental time travel was in an advanced stage.

MTT might not be unique to us, but we have the highest physical-technical accuracy and focus in our travels. And, we are probably also the species most dependent on MTT - the world in which we can survive is a world manipulated and constructed by complex artifacts, so our mind must be highly tuned to these aspects. This might be the prime reason for why we constantly observe MTT in Homo sapiens.

\section{Empirical evaluation of mental time travel}

\section{DOI: $10.1017 /$ S0140525X07002166}

\section{Caroline Raby, Dean Alexis, Anthony Dickinson, and Nicola Clayton}

Department of Experimental Psychology, University of Cambridge, Cambridge, CB2 3EB, United Kingdom.

nsc22@cam.ac.uk ad15@cam.ac.uk dma32@cam.ac.uk

crr29@cam.ac.uk www.psychol.cam.ac.uk/ccl/

Abstract: Although the mental time travel (MTT) hypothesis provides a rich, conceptual framework, the absence of clear, empirically tractable, behavioural criteria for determining the capacity for MTT restricts its usefulness in comparative research. Examples of empirical criteria for evaluating MTT in animals are given. We also question the authors' evaluation of semantic foresight and their even-handedness in assessing human and nonhuman behaviour.

Suddendorf \& Corballis (S\&C) have done a great service to the scientific community by introducing the concept of mental time travel as an integrated process in their 1997 paper (see Suddendorf \& Corballis 1997) and in restating it now in the present article. This concept has provided a background theoretical framework for our own research on memory and future thinking in animals, which we would like to acknowledge.

Here S\&C make a comparative hypothesis between the abilities of human and nonhuman animals with respect to mental time travel. However, they do not articulate this hypothesis in terms of specific behavioural criteria; and without such criteria, it is not possible to empirically evaluate their claim that mental time travel is unique to humans, because, in the absence of language, the cognitive capacities of animals can only be inferred from their behaviour. Some of the components of mental time travel (MTT) that S\&C identify in their theatre metaphor, for example, autonoesis and a sense of subjective time, are intrinsically phenomenological and hence their presence or absence is impossible to demonstrate in a nonhuman animal. Other criteria such as recursion or secondary representation may be possible to test empirically in other contexts but not, we think, in considering behaviour demonstrating mental time travel. We also question 
whether all the criteria listed - recursion, causal understanding, and rehearsal, for example - are essential for mental time travel as opposed to being skills that humans have and use incidentally while engaged in MTT.

The type of criteria we have in mind can be illustrated by our own analysis of "episodic-like" memory. We used Tulving's original definition of episodic memory in terms of spatio-temporal relations (Tulving 1972) to investigate whether western scrub jays have "what, where, when" (www) memory. We subsequently proposed additional behavioural criteria for "episodic-like" memory; namely, that the memory must not only be shown to have www content but also to have an integrated structure and that the information can be deployed flexibly (Clayton et al. 2003). Although our term "episodic-like" acknowledges the difficulty of interpreting behaviour in another species as a manifestation of a specific phenomenological experience of remembering, the important point here is that the what, where and when must be bound together in order that the animal can discriminate one event from another. For this reason even a 'yes' in every box in Table 1 of the target article is not sufficient to establish the episodic-like character of memory in the absence of the criteria of integration and flexibility.

Importantly, S\&C do offer an empirically tractable criterion for foresight, the Bischof-Köhler hypothesis. This is the claim that an animal cannot take action for a future motivational state, and we have recently used this criterion to evaluate the MTT hypothesis by investigating whether scrub-jays who are not hungry cache more food in a place in which they are likely to experience hunger the following morning than in a place that they are not. Not only did they meet this criterion of MTT, but they also cached more of a type of food that they will not receive in a particular place the following morning than of a type of food that they will (Raby et al. 2007). In Shettleworth's view this is the first unambiguous example of future planning in an animal (Shettleworth 2007).

What we do not know, and think is not testable empirically, is whether this type of future planning depends upon episodic personal projection or semantic knowledge. S\&C are inclined to dismiss the semantic cognitive system as self-evidently inferior and more limited than the episodic cognitive system with a number of statements that there is no room to debate here. A contrasting view is that the only distinguishing difference between episodic and semantic memory is the phenomenological sensation of remembering rather than knowing about the event. The semantic memory system allows individuals to acquire information about themselves and their world through different sensory modalities and flexibly retrieve and use this information. This includes personal information and autobiographical facts. We agree with S\&C that there is evidence that the episodic and semantic memory systems are closely linked to their respective future thinking counterparts and suggest that the only significant difference between episodic and semantic future thinking is the sensation of pre-experiencing one's own personal future. Humans may always combine episodic and semantic future thinking in assessing alternatives, but this does not prove that the episodic element is essential for future thinking. Using one of their own examples, it is quite possible to semantically arrange a seating plan for a forthcoming event without needing mental time travel to envisage oneself at the table.

$\mathrm{S} \& \mathrm{C}$ argue that a demonstration of mental time travel in nonhuman animals must exclude behaviours that are species typical or domain specific, without adequately explaining why. They describe a possible test of episodic-like prospection in scrubjays and then immediately dismiss it as explicable by "predispositions and specific learning algorithms," although, in their view, the use of language by humans - surely a case of predisposition and learning if ever there was one - "provides the clearest evidence of [mental time travel]." Most behaviours in any animal, cognitive or otherwise, are likely to be species typical and a result of some form of predisposition and learning. They use a different yardstick in assessing children than in assessing animals. Caching and retrieval may develop in a predictable fashion in scrub-jays, but so does the emergence of autonoesis, theory of mind, and indeed the episodic cognitive system in children.

In relying so heavily on phenomenology, S\&C effectively define mental time travel by the exclusion of animals other than humans. If they are going to propose a comparative hypothesis it is incumbent on them to offer relevant, applicable, behavioural criteria which are empirically tractable. In the absence of such criteria, the comparative aspect of MTT is not a matter of scientific enquiry but of intellectual preference.

\section{On the constructive episodic simulation of past and future events}

\author{
DOI: $10.1017 /$ S0140525X07002178
}

\section{Daniel L. Schacter and Donna Rose Addis}

Department of Psychology, Harvard University, Cambridge, MA 02138.

dls@wjh.harvard.edu http://www.wjh.harvard.edu/ dsweb

daddis@wjh.harvard.eduｈttp://www.wjh.harvard.edu/ daddis

Abstract: We consider the relation between past and future events from the perspective of the constructive episodic simulation hypothesis, which holds that episodic simulation of future events requires a memory system that allows the flexible recombination of details from past events into novel scenarios. We discuss recent neuroimaging and behavioral evidence that support this hypothesis in relation to the theater production metaphor.

Suddendorf \& Corballis (S\&C) could hardly have asked for a better moment to focus on mental time travel. In recent months there has been a virtual explosion of relevant papers, including neuroimaging studies (Addis et al. 2007; Szpunar et al. 2007), investigations of amnesic patients (Hassabis et al. 2007) and scrub jays (Raby et al. 2007), a concept essay (Schacter $\&$ Addis 2007b), and two theoretical reviews (Buckner \& Carroll 2007; Schacter \& Addis 2007a). The future of thinking about the future appears to be now.

We focus on issues raised by $\mathrm{S} \& \mathrm{C}$ that are addressed by our recent work on past and future events. In particular, we consider two key ideas contained in S\&C's theater production metaphor: (1) memory is not a literal recording of experience; and (2) episodic future thinking - like episodic remembering - needs to be decomposed into constituent components.

S\&C emphasize, rightly, that memory is constructive rather than reproductive. Though not a new idea, its links to futurerelated thinking have been overlooked (for exceptions, see Dudai \& Carruthers 2005b; Suddendorf \& Corballis 1997). To strengthen the link, we have advanced a constructive episodic simulation hypothesis (Schacter \& Addis 2007a; 2007b). According to this hypothesis, remembering past events and imagining or simulating future events draw on similar kinds of information in episodic memory and involve many shared processes. In particular, episodic remembering and future thinking both depend critically on relational processes that link or bind together distinct elements of an experience. This latter idea is especially important because our hypothesis holds that episodic simulation of future events requires a system that allows the flexible recombination of details from past events into novel scenarios. Episodic memory possesses exactly these characteristics, which makes the system highly adaptive for simulating novel future scenarios based on past experiences, but also makes the system, when used for remembering, prone to errors and distortions that arise from miscombining stored elements - a common form of memory distortion.

This hypothesis predicts considerable overlap in the processes that support remembering the past and imagining the future. 
$\mathrm{S} \& \mathrm{C}$ review such evidence, which is bolstered further by the recent studies noted earlier. For example, two new neuroimaging studies (Addis et al. 2007; Szpunar et al. 2007) build on earlier work from Okuda et al. (2003) by demonstrating that a number of prefrontal, medial temporal, and parietal regions previously associated with episodic remembering show similar increases in activity, relative to control tasks, when imagining the future or recollecting the past. A key finding with important implications for the constructive episodic simulation hypothesis is that the hippocampus shows increased activity when people construct and elaborate on both future and past events; indeed, the right hippocampus shows greater activity during construction of future than past events (Addis et al. 2007). Given the role of the hippocampus in supporting relational processing and perhaps other aspects of episodic memory, these data suggest that episodic simulation of future events involves an even more intense constructive process than does episodic remembering of past events.

Such observations mesh nicely with the recent findings from Hassabis et al. (2007) that four of five hippocampal amnesics exhibited deficits in the ability to imagine novel events. The fMRI findings also fit with a recent behavioral study in which we demonstrated that older adults, compared with younger adults, show reduced specificity both in their recall of past autobiographical episodes and their imaginings of possible future episodes (Addis et al., in press).

S\&C's theater production metaphor highlights the need to decompose the complex activities of mental time travel into more basic components. Such componential approaches have been usefully applied to memory by numerous investigators, and should be similarly helpful when applied to future event simulation. Consider, for example, the finding from our fMRI study that several brain regions show greater activity during future versus past event construction (Addis et al. 2007). As noted earlier, one of those regions is the right hippocampus. Another such region is a medial part of right anterior prefrontal cortex (BA 10). If we think of these regions as components a network used for past and future event simulation, the next task is to characterize their respective contributions. In the context of S\&C's theater metaphor, we suggest that medial BA 10 , assisted by hippocampus, might serve as the "stage" on which the production unfolds.

S\&C discuss the stage component with respect to Baddeley's well-known tripartite model of working memory, consisting of a central executive, phonological loop, and visuo-spatial sketchpad. Note, however, that Baddeley (2000) updated the model to include a fourth component, the episodic buffer. In the updated model, the central executive is associated with strategic control of processing, whereas the episodic buffer is "a limited capacity system that provides temporary storage of information held in a multimodal code, which is capable of binding information from the subsidiary systems, and from long-term memory, into a unitary episodic representation" (Baddeley 2000, p. 417).

The function of binding multimodal information from diverse systems makes the episodic buffer well suited to serving as a stage for future event simulations. FMRI data from a working memory task that requires integration of spatial and verbal information indicate that medial BA 10 is a candidate site of the episodic buffer, because it is preferentially activated during maintenance of integrated versus unintegrated information (Prabhakaran et al. 2000). Given the proximity of this region to the one in which we documented greater activity for future imagining than remembering, we are encouraged to postulate that medial BA 10 holds together the diverse elements compromising a future event simulation, and may work closely with the hippocampus in constructing the elements that support the full-blown theater production. Exploring the interrelations of these regions constitutes a critical next step for future-oriented research.
ACKNOWLEDGMENTS

Preparation of this paper was supported by grants from the NIA and NIMH to DLS. We thank A. Wong for assistance and M. Bar for comments.

\section{Studying mental states is not a research program for comparative cognition}

\author{
DOI: $10.1017 /$ S0140525X0700218X
}

\section{Sara J. Shettleworth}

Department of Psychology, University of Toronto, Toronto, Ontario M5S 3G3, Canada.

\section{shettle@psych.utoronto.ca}

Abstract: The title of the target article suggests an agenda for research on cognitive evolution that is doubly flawed. It implies that we can learn directly about animals' mental states, and its focus on human uniqueness impels a search for an existence proof rather than for understanding what components of given cognitive processes are shared among species and why.

"What is mental time travel, and is it unique to humans?" As the focus for research on cognitive evolution, this question is doubly flawed. Not only can we never know what other species' mental experiences are like, searching for a yes or no answer to a question about human uniqueness is not a productive way to proceed with research in comparative cognition. This path leads to a quest for an existence proof (just one animal with "it" is enough), followed by endless disputes over whether "it" really was demonstrated. The history of attempts to teach forms of human language to apes (Shettleworth 1998) is evidence enough for this. Far more productive as well as consistent with evolutionary thinking is to ask something like, "What components of process $\mathrm{X}$ are and are not shared among what species and why, in evolutionary, functional, and perhaps neurological terms?" Progress in answering such questions is most likely to be made when the process being compared across species is already well understood within at least one of the species in question, human or other animal.

In various places, Suddendorf \& Corballis $(\mathrm{S} \& \mathrm{C})$ do address the difficulty of drawing conclusions about animals' mental experiences, for example, when discussing episodic-like memory in section 3.1. But this is not merely a matter of not having enough evidence yet: Because we can only observe behavior, we can never know if animals are mentally time traveling anywhere. Researchers can productively seek no more than functional similarities between human and animal behaviors in analogous situations. A pattern of brain activity ( $\& \& C$, sect. 2.3) is just another item of function similarity, and one that is a priori denied to species with very different brains from humans.

An instructive example of what is meant by documenting functional similarity of behaviors is research on metacognition in rhesus macaques. Contrary to what S\&C suggest in section 4.5, there is a now a rich body of convergent evidence from several laboratories and testing paradigms consistent with these monkeys' being able to monitor both the certainty of their perceptual judgments and the strength of their memories. For example, when memory is poor, monkeys choose to escape tests of memory more often; but when they choose to take the test, they continue to perform well, better than when forced to take it (Hampton 2001; see also Smith \& Washburn 2005). Monkeys show this functional relationship immediately when memory is taxed, further indicating that their behavior does not reflect learning to use cues like length of the retention interval. Monkeys' reports of metacognitive certainty transfer across tasks (Kornell et al. 2007), and they behave as if they are using it to control information seeking in naturalistic (Hampton et al. 2004) and operant tasks (Kornell et al. 2007). The availability of multiple convergent measures and more than one testing paradigm also 
renders meaningful repeated failures to find similar patterns of data in pigeons (Sutton \& Shettleworth, in press).

One reason this body of work is so compelling is that there is reasonable theoretical agreement on relevant procedures and predicted patterns of data. The tests for animals are also closely analogous to those used with people, and indeed data from people and monkeys have sometimes been reported in the same article (see Smith et al. 2003). As alluded to by S\&C, in some of these situations, rather than explicitly "reporting on" memory strength, the monkeys may be discriminating some self-generated correlate of memory strength such as latency to select an option; but the same may be true of people (Terrace $\&$ Metcalfe 2005). Such possibilities remain for further study. Nevertheless, among attempts to document behaviors in animals functionally similar to those accompanied by distinct kinds of awareness in humans, research on monkey metacognition is unique in richness and diversity of convergent data. Even so, it still cannot reveal the monkeys' subjective states.

S\&C do try to specify (in sect. 2.1) what nonverbal behavior would count as evidence of "future mental time travel." It should be a novel behavior or combination of behaviors, the outcome of which later satisfies a need not present at the time of performance. They then suggest an experiment very similar to one recently reported by Raby et al. (2007) with positive results. But, as S\&C say, an additional requirement that follows from the folk-psychological notion of human planning is that the ability revealed in such a test be flexible, that is, applicable in more than one context. It remains to be seen whether this new study with scrub jays has tapped a generalized ability or a hitherto unrecognized learning adaptation in the food-caching system (Shettleworth 2007).

S\&C also propose separable components of mental time travel that might be sought in developing children and/or other species. This approach to comparative research is undoubtedly useful in general. For example, the comparative study of numerical cognition has made real progress since researchers moved beyond asking, "Do animals count?" (see Shettleworth 1998) to the comparative analysis of components of numerical competence such as exact perception of small numerosities and Weber's law-based discrimination among larger ones (Feigenson et al. 2004). These may be widely shared among vertebrates, whereas exact appreciation of quantities larger than about 5 is normally confined to numerate humans (Pica et al. 2004). In contrast, the components of mental time travel proposed by S\&C seem too many and too vaguely metaphorical for similar progress in this area to be expected any time soon. It is abundantly clear (Roberts 2002; Shettleworth 1998) that all animals are superbly equipped with learning and timing mechanisms allowing them to use their own and their ancestors' past to determine future behavior. Interpreting what they do as revealing mental time travel is one example of a rather widespread return of anthropomorphism, which appeals to the public without necessarily advancing understanding (Wynne 2007). Perhaps there should be a moratorium on studies of animal "mental time travel" until its psychological and behavioral components have been better specified and more carefully dissected in developmental studies.

\section{First test, then judge future-oriented behaviour in animals}

\section{DOI: 10.1017/S0140525X07002191}

\author{
Elisabeth H. M. Sterck ${ }^{\mathrm{a}, \mathrm{b}}$ and Valérie Dufour ${ }^{\mathrm{b}, \mathrm{c}}$ \\ ${ }^{a}$ Department of Behavioural Biology, Utrecht University, $3584 \mathrm{CH}$ Utrecht, \\ The Netherlands; 'bthology Research, Animal Science Department, \\ Biomedical Primate Research Centre, 2288 GJ Rijswijk, The Netherlands; \\ 'Institut Pluridisciplinaire Hubert Curien, UMR 7178, Department Ecologie, \\ Physiologie and Ethologie, CNRS, 67087 Strasbourg, Cedex 02, France.
}

E.H.M.Sterck@bio.uu.nl http://www.bio.uu.nl/behaviour/Sterck/index.html Valerie.dufour@c-strasbourg.fr

Abstract: Suddendorf \& Corballis (S\&C) argue that animals are not capable of mental time travel (MTT) or its components. However, new results on chimpanzees suggest that they plan for the future and possess some MTT components. Moreover, future-oriented behaviour and episodic-like memory in other animals suggest that not all animals are limited to the present. Animals' capacities should not be dismissed without testing them.

"Darwinian continuity need not demand greater mental powers in nonhuman animals than is currently evident" (target article, sect. 6, para. 10). With this closing sentence, Suddendorf \& Corballis (S\&C) seem to dismiss the possibility that nonhuman species may mentally travel in time or possess any of its components. However, their approach to mental time travel (MTT), distinguishing different cognitive components, that is, the "play" (or theater) metaphor, actually allows for a Darwinian continuity without MTT in animals. Moreover, the conditions for MTT in animals are laid out, so the capacity of MTT itself can be investigated. Our research on future-oriented behaviour in chimpanzees (Dufour \& Sterck, submitted; Dufour et al. 2007), and recent studies on other apes (Mulcahy \& Call 2006) and birds (Raby et al. 2007), indicate that these animals can plan for future needs and have some of the mental capacities required for MTT.

New research indicates that flexible future-oriented behaviour in animals exists. Animals show future-oriented behaviour when they act in the present on the basis of an anticipated future need in contrast to a current one. The results of the sole study on apes showing such planning behaviour in tool use tasks (Mulcahy \& Call 2006) are not considered convincing by S\&C. They argue that the apes "only" needed to learn to always return with the same tool. Moreover, the set-up allowed for tool collection based on a current need since the apparatus was visible. Our results on chimpanzees, however, counter this interpretation. We replicated the Mulcahy and Call study with additional methodological controls: Chimpanzees had to collect the appropriate tools and bring them back while the apparatus and experimenter remained out of sight. Some individuals transported the appropriate tools (Dufour \& Sterck, submitted). Moreover, the animals had not previously succeeded in a planning-like task that required them bring in a tool, and also never had to bring the tool during training, so a previously learned rule cannot account for their behaviour. Therefore, our study indicates that chimpanzees can collect tools without direct indications of future use (Dufour \& Sterck, submitted).

Similarly, scrub jays can store their breakfast in the evening at a location where they occasionally are forced to spend the night, but are never provided with breakfast, suggesting future-oriented behaviour not driven by current need (Raby et al. 2007). Although more controls are needed, these results are first indications that animals, too, may possess capacities related to MTT. In addition, planning for the future and recollection of past experiences are probably linked (e.g., Suddendorf 2006). An increasing body of research concerns recollection of past events, or episodic-like memory, in animals, reviewed by S\&C (sect. 3.1). Although they conclude that animals lack MTT, their review shows that rodents, monkeys, great apes, and scrub jays can associate two or more elements (what, where, when, who) from a past experience and combine them to direct current behaviour. Altogether, these results indicate that not all animals are strictly bound to the present.

S\&C list in the "play" metaphor a challenging range of mental capacities that are components of MTT. From the capacities mentioned, the "broadcaster" is probably not required for MTT, although it may enhance joint action. In addition, not all capacities may be required to the degree proposed by $\mathrm{S} \& \mathrm{C}$.

S\&C suggest that animals must possess some "appreciation of the time dimension itself" (sect. 4.4), that is, "the set." Most tests of time understanding in animals show the limited capacities of 
monkeys, rats, and pigeons in choosing a delayed large reward over a directly available small one. However, we showed that chimpanzees may appreciate exactly this time dimension. In a "waiting to exchange task," we determined the capacity of capuchin monkeys and apes to wait (Dufour et al. 2007; Ramseyer et al. 2006). Animals were given an initial item of a desired food (a cookie) that they had to keep for some time and exchange later for a larger-sized cookie. While capuchins did not wait for more than 20 to 40 seconds, chimpanzees kept the initial cookie for at least 8 minutes. Interestingly, chimpanzees gave up waiting earlier than predicted by their general ability to wait. This suggests some appreciation of the duration of the delay, and a flexible capacity of weighing different options based on time to and size of what is to come relative to what is currently available. Chimpanzees appreciate the time dimension for a time period exceeding that of other tested animals, and their performance resembles that of children (Mischel et al. 1989).

The results of the "waiting to exchange task" are also relevant for the "executive producer" capacity. They indicate that chimpanzees can "[inhibit] a simple response in order to increase" their "total future reward" (sect. 4.6). In addition, chimpanzees had voluntary control over this inhibition, since, with the longer waiting times, they either decided to control themselves or chose not to.

In "the actors" part, the ability to project self into the past or the future, MTT requires self-awareness. S\&C imply that theory of mind (ToM) is a prerequisite to MTT. However, ToM is also considered an advanced cognitive capacity often attributed to humans only. Similar to what S\&C propose, ToM has also been proposed to require a number of components, among them the capacity to appreciate the future (Barrett et al. 2003, p. 496). This suggests that one is not part of the other, since they are two different capacities, but that both MTT and ToM depend partly on the same components.

In conclusion, our results on future-oriented behaviour in chimpanzees indicate that they possibly show future planning and have some of the mental capacities required for MTT. The search will be on for the others. As S\&C also acknowledge, before accepting the conclusion that "Darwinian continuity need not demand greater mental powers in nonhuman animals than is currently evident," we have to test animals before dismissing their MTT-related capacities.

ACKNOWLEDGMENTS

We thank Marusha Dekleva and Matt Bruce for comments on earlier versions of the paper.

\section{The medium and the message of mental time travel}

DOI: $10.1017 /$ S0140525X07002208

\section{Endel Tulving and Alice Kim \\ The Rotman Research Institute, Baycrest Hospital, Toronto, M6A 2E1, Canada. \\ tulving@psych.utoronto.ca aa.kim@utoronto.ca \\ http://rotman-baycrest.on.ca/index.php?section $=219$}

\begin{abstract}
We add one point to Suddendorf \& Corballis's (S\&C's) story of mental time travel: For the future success of this hot, new area of research, it is imperative to pay attention to the fundamental distinction between the general brain/mind capacity that makes possible conscious awareness of the past and the future (the "medium"), on the one hand, and specific expressions of this capacity in a large variety of future-related mental activities (the "message"), on the other.
\end{abstract}

"Oh, grandmother, what big teeth you have!" "All the better to eat you with, my dear."
Things always seem to go more smoothly in fairy tales than in real life. Little Red Riding Hood receives a straight answer to her simple remark. But when scientists say, "Oh, Mother Nature, what big brains you have given us," Mother Nature only smiles, "All the better for you, my dear, to figure out my ways." Perhaps.

Despite the spectacular advances that science has made, we still do not know why the human brain is so much bigger than the chimp's. Surely it has evolved to serve some adaptive functions that are not shared by our smaller-brained relatives, but, apart from language, it is not obvious what these functions might be. Interestingly, the difficulty of the problem itself may provide an important clue to the solution of the puzzle: Whatever these uniquely human functions are, they must be so subtle that even clever scientists do not see them. In other words, the clue is to look for differences between humans and nonhumans that are not obvious.

Nothing can be more subtle than the ability to reflect on what one has experienced in the past or might experience in the future. Although this ability has existed since time immemorial, until recently no one has paid much attention to it as an object of scientific interest. Now, however, mental time travel, the topic of Suddendorf \& Corballis's (S\&C's) target article, is moving to the center stage of brain/mind sciences with a vengeance. During the time alone that the $\mathrm{S} \& \mathrm{C}$ article spent in the publishing pipeline, a whole slew of prominent papers on mental time travel have appeared in leading journals (Addis et al. 2007; Buckner \& Carroll 2007; D’Argembeau \& Van der Linden 2006; Schacter \& Addis 2007b; Spreng \& Levine 2006; Szpunar et al. 2007). There is every reason to believe that it is going to be among the hot topics of the future. Who can resist the allure of the idea of a universal yet invisible human trait?

We like the general thrust of S\&C's story, as well as their novel attempt to decompose mental time travel into more basic components. This is the way of healthy science. We agree with many details of their argument. Specifically, we concur with their judgment that nonhuman animals probably do not possess the same kind of ability for mental time travel that humans do. Apart from the fact that, as yet, no convincing evidence of this ability has been shown by any creature other than humans, there are at least three other reasons why we side with S\&C on this issue: (1) It helps with the puzzle of the large human brain. (2) The view opposing it helps one understand why mental time travel, until recently, had not been singled out for study. (3) It helps provide explicit guidance in the search for the neural underpinnings of mental time travel.

If we were permitted to offer one suggestion to $\mathrm{S} \& \mathrm{C}$ - and others who are or will be studying mental time travel - it would be to pay attention to the relevant concepts and their attendant terminology. In the forthcoming debate about mental time travel, arguments should be about facts and their interpretation, and not the words used to exchange ideas about them.

What exactly is mental time travel? What do we mean when we use the term? In the S\&C story, the term "mental time travel" is used to refer to an evolved faculty or capacity, that "allows us not only to go back in time, but also to foresee, plan, and shape virtually any specific future event" (target article, Abstract) and thereby enhances flexibility in future-related behavior. At the same time, however, it is also used to refer to the kind of mental activity that it allows or enables, for example "anticipation of future needs" (sect. 3.3). Now, a capacity that allows or enables a particular activity is not the same as the activity itself. The distinction may be subtle, but it is fundamental, and it should be reflected in the terms that are used to refer to them.

The distinction between general capacity and particular behavioral/cognitive expressions of it may become especially relevant in the investigation of neural correlates of mental time travel, as illustrated by recent functional neuroimaging studies. In these studies, the temporal aspects of the mental time travel capacity (past vs. future) have been confounded with the details of the nature of the imagined activity: As a consequence of the employed tasks, what people imagine for future events is 
necessarily different from what they remember about past events. Ideally, and eventually, such confounding will be eliminated, and the neural correlates of the neurocognitive capacity that enables mental time travel (the "medium") will be separated from the neural correlates of the contents of what is imagined or remembered (the "message").

Our own terminological preference is to reserve the term "mental time travel" for the kinds of mental activity in which people engage when they remember particular past events or think about possible personal future happenings, and to use some other term, such as "chronesthesia" (Tulving 2002a), to refer to the general neurocognitive capacity that makes such remembering and thinking possible. The concept of chronesthesia was introduced as a specifically time-oriented facet of autonoetic consciousness. The concept of autonoetic consciousness was introduced as a part of the description of a brain-damaged amnesic individual who could neither remember any personal past happenings nor imagine any personal future scenarios (Tulving 1985). This patient was said to have lost his autonoetic consciousness, a kind of consciousness that endows a healthy person with the capability of mental time travel, to "roam ... at will over what has happened as readily as over what might have happened, independently of the physical laws that govern the universe" (Tulving 1985, p. 5).

If the Bischof-Köhler hypothesis survives the challenges from clever tiny-brained birds and their even cleverer keepers (e.g., Raby et al. 2007), it will make sense to look for some unique properties - cytoarchitectonic, hodological, neurochemical - in the human brain. S\&C mention the possible usefulness of this physiological criterion, and we think the optimism in this regard is well placed.

So, is the large human brain relevant to the story? Nobody knows at this stage; time will tell. But the world is full of testimony that larger, more recently evolved systems can perform feats that smaller, more ancestral ones cannot. If Mother Nature wants to hide her ultimate secrets, what better place for it than a humongous brain, the most elaborate structure known in the universe.

\section{Authors' Response}

\section{Mental time travel across the disciplines: The future looks bright}

\author{
DOI: $10.1017 /$ S0140525X0700221X
}

\section{Thomas Suddendorf $^{\mathrm{a}}$ and Michael C. Corballis ${ }^{\mathrm{b}}$}

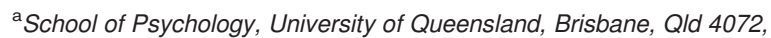
Australia; ' Department of Psychology, University of Auckland, Auckland 1142, New Zealand.

t.suddendorf@psy.uq.edu.auｍ.corballis@auckland.ac.nz

Abstract: There is a growing interest in mental time travel in cognitive psychology, neuroscience, developmental psychology, comparative psychology, and evolutionary psychology. Here we review current issues in each of these disciplines. To help move the debates forward we name and distinguish $15 \mathrm{key}$ hypotheses about mental time travel. We argue that foresight has for too long lived in the shadows of research on memory and call for further research efforts.

\section{R1. Introduction}

The fundamental human capacity to conceive of future events has received surprisingly little attention in the behavioral and brain sciences. Ten years ago we first discussed the nature, development, and evolution of mental time travel in a rather low-key monograph (Suddendorf \& Corballis 1997). That article was based on Suddendorfs masters thesis (Suddendorf 1994), and it took much persistence to get it published after initial rejections from several journals, including this one. It is thus a delight to see how far we have come, as is evident in the wide interest the target article elicited. Today, mental time travel is recognized as an important topic of inquiry with discussions in the top generalist and specialist journals. In fact, since writing this target article last year, there has been, as Schacter \& Addis, Tulving \& Kim, and Okuda note, an explosion of articles published on various aspects of the subject. We look forward to reviewing progress in another 10 years' time, though extrapolating from the recent surge in interest, that task will probably be a lot more difficult.

We thank the commentators for providing their perspectives and will reply according to the five main research areas from which they derive: cognitive psychology, neuroscience, developmental psychology, comparative psychology, and evolutionary psychology. This division should make it easier to find the relevant sections for scholars mainly interested in some of these areas.

Tulving \& Kim counsel that researchers should carefully distinguish concepts and terminology in the forthcoming debate. To avoid confusion, we therefore begin our reply by explicitly naming and distinguishing various hypotheses from the target article that attracted comment (see Table R1). Many of the commentaries take issue with only one of these and may not bear on others.

\section{R2. Mental time travel and cognitive psychology}

The study of memory has always been a cornerstone of cognitive psychology. It remains something of a mystery, though, why memory research has attracted so much more attention than the study of future-oriented processes. One reason, we suspect, has to do with the ease of testing memory accuracy and the difficulties associated with testing foresight. We have argued that an evolutionary perspective must shift the focus to what these capacities can do for the present and the future, and the commentaries offer strong support for the Future-first hypothesis (but see Nelson). In fact, it seems that the future of thinking about the future is now, as Schacter \& Addis put it. We are delighted to read that memory researchers such as Tulving \& Kim and Schacter \& Addis fundamentally agree with our proposal and endorse a fresh look at foresight and how memory may support it.

There is almost unanimous agreement on the general hypothesis that there are some fundamental links between mental time travel into the past and the future (Janus hypothesis). Several commentators substantiate this claim with further evidence (Brüne \& BrüneCohrs; Buckner; Carr \& Viskontas; D’Argembeau \& Van der Linden; Hurford, Flaherty, \& Argyropoulos (Hurford et al.); Okuda; Schacter \& Addis). Nonetheless, it is probably prudent to remain cautious about the extent of these links, as Friedman suggests. The details of the relation are still to be determined (Bar) and we 
Table R1. Key hypotheses discussed in the target article

Hypothesis Name

1. The Janus hypothesis

2. The Prospection systems hypothesis

3. The Components hypothesis

4. The Traveler hypothesis

5. The Theory-of-mind hypothesis

6. The Theater hypothesis

7. The Future-first hypothesis

8. The Constructive episodic simulation hypothesis

9. The Prime mover hypothesis

10. The Flexibility hypothesis

11. The Uniqueness hypothesis

12. The Stuck-in-time hypothesis

13. Bischof-Köhler hypothesis

14. The Multiple limits hypothesis

15. The Lower Paleolithic emergence hypothesis
Core Idea

Mental time travel into the past and future are closely linked in mind and brain.

Procedural, semantic, and episodic memory systems have direct prospective counterparts.

Mental time travel is not an isolated module, but draws on a set of other cognitive capacities.

Mental time travel depends on autonoetic awareness and concepts of self and time.

Mental time travel draws on many of the same mechanisms as theory of mind.

Successful mental time travel depends on a set of components that fill the metaphorical roles of stage, playwright, actors, set, director, producer, and broadcaster.

Episodic memory is an adaptive design feature of the human ability to conceive of future events.

Characteristic errors in episodic memory reflect a construction process designed to simulate future events.

Mental time travel was a driving force in human evolution.

The key adaptive advantage of mental time travel is greater behavioral flexibility and control over future survival and reproduction chances.

There is something about human foresight that no other species shares.

Nonhuman animals cannot travel mentally in time.

Nonhuman animals cannot anticipate future need/drive states that they currently do not experience.

Nonhuman animals' foresight is restricted by deficits in any one, or any combination, of the components of the theater hypothesis.

Mental time travel first emerged in the genus Homo.
References (other than target article itself)

(Dudai \& Carruthers 2005a; Schacter \& Addis 2007b; Suddendorf \& Busby 2003; 2005; Suddendorf \& Corballis 1997; Tulving 1985; 2005)

(Suddendorf \& Corballis 1997)

(Tulving 1985; 2005)

(Hazlitt 1805; Perner \& Ruffman 1995; Suddendorf 1999; Suddendorf \& Corballis 1997)

(Suddendorf \& Busby; 2003; 2005; Suddendorf \& Corballis 1997)

(Schacter \& Addis 2007a; Suddendorf \& Busby 2003; Suddendorf \& Corballis 1997)

(Suddendorf 2006; Suddendorf \& Corballis 1997)

(Suddendorf \& Corballis 1997)

(Suddendorf \& Corballis 1997)

(Roberts 2002; Suddendorf \& Corballis 1997; Tulving 1983)

(Bischof-Köhler 1985; Bischof 1978; Suddendorf \& Corballis 1997)

(Suddendorf \& Corballis 1997) 
suspect that both shared and unique processes for episodic memory and foresight will be established. None of the commentators made the case that mental time travel might be a separate module. Instead, in addition to episodic memory providing raw material for mental simulation of future events, the link may be based on both past and future travels drawing on the same cognitive resources (Components hypothesis). We suggested a range of components with the Theater hypothesis.

\section{R2.1. Theatrical concerns}

Although generally commending the Theater hypothesis, Hurford et al. query the role of the broadcaster (see also Sterck \& Dufour). By adding the broadcaster to the metaphor, they suggest, we preclude possible research on episodic memory in nonhumans. The broadcaster can be most clearly identified with language, but we did mention the possibility that nonhuman animals might in principle be able to communicate past episodes (e.g., by using mime). In any case, we did not mean to imply that the broadcaster was a necessary component of the theater metaphor; we wrote, "Although language is not necessary for mental time travel, it provides the clearest evidence of it" (sect. 4.7).

The causal relation, though, may run the other way. That is, mental time travel may have been critical to the evolution of the broadcaster. Indeed, as we hinted in the target article, language seems exquisitely adapted to the description of events that are remote in place and time. So it is that language comprises symbols to represent nonpresent objects, actions, emotions, and the like, and has systems of tense for signaling past and future (see Corballis \& Suddendorf, in press, for further discussion). It has become commonplace to refer to one component of memory as declarative memory; conversely, we suggest, much of language consists of memorial declaration. It also consists of declarations about the future, as evident in recent discussions about the consequences of global warming.

Dessalles, though, seems to think that the broadcaster may have come first, arguing that episodic memory evolved as an outgrowth of language (see also Nelson), providing us with worthwhile things to talk about. This seems to us the wrong way around, rather like suggesting that feet evolved to allow us to play football. It is surely more likely that language evolved to allow experiences and plans, and also knowledge, to be shared. Dessalles' position, moreover, appears to be somewhat circular: We can tell of events because they are tellable-hence episodic memory evolved in support of language performance. Although there may be individual differences in the extent that people disclose, we suspect that every clinically normal person has memories and anticipations that they do not share with others. In fact, we think it is at times very difficult to put into words what has happened or what we expect to happen. And although telling exciting stories of the unusual and surprising may have social (and perhaps sexual) payoffs, it surely does not stop people from remembering the mundane and even talking about it. I well remember getting on the bus last week, but it is not a story I would tell. We agree with Dessalles that this is so because it conveys little new or unexpected information. But why is unexpectedness important? Nelson claims that it is only humans who share events they have experienced. We concur, and this broadcasting of one's mental time travel can indeed be extremely adaptive, in that it allows people to learn from others' mistakes and successes without even having to experience the events themselves. Those who tell such useful tales may be encouraged and rewarded by those who learn. Thus, these are stories worth telling and worth listening to.

Whereas Hurford et al. think the Theater hypothesis goes too far, Ainslie suggests that it does not go far enough, since it neglects the audience. In so doing, it fails to consider the question of what motivates us to indulge in mental time travel rather than live in the present. Questions about motivation for mental time travel are perhaps separate from questions about mental time travel itself, just as the description of a theater production is not a description of who will go to see it. Nevertheless, Ainslie raises interesting points. He notes that people have little tolerance for a bad lecture - or reading a boring article, we might add - and it may be precisely in such circumstances that they will indulge in some future fantasy, or revel in some past glory. Buckner and Carroll (2007) suggest that this may in fact be the human default mode. Even so, different scenarios must compete with each other and with present needs. Ainslie suggests that they may compete in terms of entertainment value and suggests that a primary motivator may be the very uniqueness of scenarios that we can envisage; that uniqueness may in turn result from our ability to use combinatorial principles to generate novelty (see sect. R2.5), and also perhaps from what Locke and Bogin (2006) recently termed the "instinct for inventiveness" (see also Whiten \& Suddendorf [in press] on the evolution of inventiveness).

\section{R2.2. The role of emotion}

D’Argembeau \& Van der Linden offer some detail on the role of emotion in mental time travel. Their interesting work has documented links between past and future time travel (Janus hypothesis), adding depth to the study of its content. They suggest that in addition to enhancing future fitness, mental time travel serves to regulate emotion and create the sense of self. We suspect it has many other functions as well. In fact, many human emotions, such as remorse and hope, may fundamentally depend on mental time travel. Individual differences in the emotional content of imagined episodes may underlie various mental disorders (see also Brüne \& Brüne-Cohrs); thus depressed people generate fewer positive episodes; and anxious patients, more negative episodes (MacLeod \& Byrne 1996). On the other hand, they note that people with stronger visual imagery generate more detailed event representations and rate these as more meaningful (D'Argembeau \& Van der Linden 2006), and they ask whether this may also be associated with a stronger ability to make adaptive choices. There is reason to suspect that this might be so, as other work has found that individuals with stronger concern for the future show better health-preserving behavior (e.g., Mahon et al. 1997). However, this comes at a price, as they are also more prone to stress (Zimbardo \& Boyd 1999). As Ainslie points out, there are serious problems associated with being, like Walter Mitty, too "fantasy-prone." It is easy to see how the adaptive ability to concern oneself with the future confronts humans with all kinds of cognitive, motivational, and emotional 
complexities and dilemmas (e.g., Suddendorf \& Busby 2005). For example, one may struggle to stop thinking about the same events (as in posttraumatic stress syndrome), or worry about events that may never occur. Brüne \& BrüneCohrs make the interesting suggestion that obsessivecompulsive disorder might be the result of too much mental time travel activity of this latter kind (Brüne 2006).

Sometimes, though, the sense of time can be seen as a solution rather than a dilemma. Viola in Shakespeare's Twelfth Night, masquerading as a man, finds herself in an impossible predicament, and is moved to say

O Time, thou must untangle this, not I;

It is too hard a knot for me t'untie!

We often live in the hope that problems will simply go away.

According to Atance \& Meltzoff, present affective state is not so much a consequence of mental time travel as a cause of it. They suggest that mental time travel arises because we experience some desire for a future goal in the present, and work toward it by constructing future scenarios. This is similar to the point raised by Ainslie that we need to consider what motivates a person to indulge in future thinking in the present, or to think about one scenario rather than another. Emotional considerations are no doubt important (e.g., D'Argembeau \& Van der Linden), but it is not clear to us that mental time travel is always suffused with emotion, and the role of emotion in memory has a controversial history. Some of the things we remember seem banal and without obvious affect (see also our response to Dessalles), and they may serve more as part of the vocabulary from which to construct future scenarios.

\section{R2.3. About time}

Hurford et al. suggest what they call a "radically different" taxonomy from the Prospection-systems hypothesis we summarized in our Figure 1, although their alternative ideas do not really impinge on the notion of mental time travel itself. They see no distinctions between semantic memory and (semantic) prospection, or between procedural memory and procedural prospection, since both semantic and procedural memory are essentially timeless. Semantic memory is indeed timeless in the sense that it does not record and allow us to re-experience a specific event that occurred at a specific point in time, but we can of course have semantic memories that refer to time. We can know, for example, when we were born, or the date at which Columbus discovered America. Further, some semantic knowledge is clearly directed toward the future, such as knowledge of an upcoming birthday party, and influences our planning as much as the episodic constructions that may guide the specific form of the celebrations. As we noted in the discussion of the reminiscence bump in the target article, with increasing age we may rely less on mental time travel and increasingly on the semantic system. The notions of past and future can be embedded in semantic knowledge as well. We suggest, though, that the semantic representation of time itself depends on the initial emergence of mental time travel, both in evolution and development.
This possibility receives some support from Friedman, who rightly points out that our sense of the time at which events occurred in the past is fragmentary and generally based on inference rather than on direct experience (see also Eacott \& Easton). He thus suggests that episodic memory should be understood as having to do with events that have occurred on a particular occasion, rather than at a specific time. In a sense, then, our concept of time is an extrapolation from incomplete data. Nevertheless, we certainly understand time to be a continuum, running smoothly from some infinite past (or perhaps from the big bang that created the universe) to a future that is hopefully distant, global warming or not. Time is therefore woven into our semantic knowledge about the world. But such notions, we argue, can only emerge from an initial understanding of a contextualized, eventfilled time (cf. Gerrans 2007).

Merker suggests that our "entire argument is built on a perfect symmetry between past and future," despite our having written "There is a fundamental causal asymmetry [between past and future], and one simply cannot know the future as one knows the past". More seriously, perhaps, he suggests that semantic prospection is all that is needed for optimal future planning (cf. Hegdé) and asserts that we have provided no concrete evidence that mental time travel ever yields greater efficacy. As noted earlier, mental time travel may be necessary to construct such semantic prospection. Furthermore, his view of prospection seems too passive, as though mental time travel into the future is little more than a guessing game. Thus he suggests that we cannot know which one of several possible future events will actually eventuate, and that particularities gleaned from past episodes might make prediction worse by adding to the number of possible alternatives. On a more proactive view, though, those particularities can surely help us actually shape future events, as distinct from merely trying to anticipate what will happen.

\section{R2.4. Beyond past and future}

Mental time travel may be based on components that are also used to achieve other goals (the Components hypothesis). The Theory-of-mind hypothesis, for example, suggests that humans use the same mechanisms to simulate the mental states of others. This idea is not new (as Bischof-Köhler \& Bischof and Sterck \& Dufour seem to imply), but was a central argument in our original article (Suddendorf \& Corballis 1997). Other authors have also pointed to links between theory of mind and mental time travels into past (Perner \& Ruffman 1995) and future (Hazlitt 1805). Bischof-Köhler \& Bischof cite additional developmental support for this hypothesis, and make the interesting case for taking the Gestalt psychological concept of "frame-of-reference awareness" as a perspective for understanding mental time travel. Buckner and Okuda provide additional reasons why theory of mind and mental time travel (and possibly some other forms of "self-projection" such as in navigation) might draw on the same components, and none of the commentators made a case for conceiving of mental time travel as an informationally encapsulated module. In the target article, we proposed that mental time travel draws on a range of sophisticated components (the 
Theater hypothesis) that may serve a range of other cognitive functions, including theory of mind.

The relation between mental time travel and theory of mind may be quite complex. On the one hand, both may draw on basic component abilities such as metarepresentation and executive functions (e.g., Suddendorf 1999), or "frame-of-reference awareness"; on the other hand, each appears to be intimately intertwined with the other. For example, to understand what oneself and others might do in the future typically requires some appreciation of different mental states. Conversely, mental time travels are mental states, and understanding others' minds often depends on understanding what happened to them, and what their plans and goals might be. The recursion evident in theory of mind (e.g., I believe that you know what she wants) is also evident in mental time travel (e.g., I remember that I thought yesterday about what you are going to do tomorrow).

\section{R2.5. Combinations and recombinations}

Although we proposed that past episodes provide a vocabulary from which to construct possible future episodes, using recursive, combinatorial principles similar to those involved in theory of mind and language, Hegdé misinterprets us as implying that "one can mentally create only those future events that one has specifically experienced in the past." Like Merker, Hegdé also argues that the information provided by episodic memory is not needed and can even place "untenable limitations on one's ability to imagine." He suggests that prospection is most efficiently accomplished entirely through the use of Bayesian principles (which he does not specify) based on generic prior knowledge, although he does concede that the Bayesian approach can use episodic memory without being dependent on it. We agree that imagining future episodes is based on both semantic knowledge and episodic memory, but suggest that Hegdé may have underestimated the constructive, combinatorial aspect of our account. We recognize that humans can imagine whatever, wherever, and whenever (Suddendorf \& Busby 2003b), and we emphasized the generative nature of mental time travel both in the target article and in the original formulation:

One characteristic of mental time travel that distinguishes it from instinct and associative learning is its flexibility. That is, given a basic vocabulary of actors, objects, and events, we can construct unique episodes in the past and create scenarios to deal with unique contingencies in the future. (Suddendorf \& Corballis 1997, p. 147)

This generativity may be an asset for flexible foresight, but it may serve less well for accurately recording events of the past, lending support to our argument (the Future-first hypothesis) that episodic memory may in fact be a design feature of the foresight system (cf., D’Argembeau \& Van der Linden). Schacter \& Addis reinforce our claim that the error-prone nature of episodic memory is the result of a system geared towards flexible recombination of future events, rather than towards faithful reconstruction of the past - and suggest we should call this idea the Constructive episodic simulation hypothesis (Schacter \& Addis 2007a). The malleability of memory that we have known of since Bartlett's (1932) pioneering studies is often forgotten by researchers who use correct recall as the dependent variable. Several commentators (e.g., Ainslie; Schacter \& Addis) agree with us that the veracity of episodic memory cannot be used to define it. Just as visual illusions hold vital clues to studying normal vision, so errors of memory also hold clues to understanding the system (see also Brüne \& Brüne-Cohrs), as is well illustrated by Schacter's (e.g., 1999) seminal work on these "sins of memory."

In the playwright section of the theater metaphor, we focused especially on the peculiar human capacity for recursion, which drives the recombination of elements not only in mental time travel but also in other spheres such as language, theory of mind, and music (Corballis 2007). This may have distracted from other aspects of the story line. Nelson therefore argues for the importance of narrative in mental time travel. We are sympathetic to her view (see also the discussion in sect. R4) and have acknowledged previously (Suddendorf \& Corballis 1997) Freud's observation that even memories that reveal themselves as images require a story grammar if remembrance is to be distinguished from random hallucinations. We thought the use of the theater metaphor itself would have gone a long way to emphasizing the importance of the story or narrative being played out.

\section{R3. Mental time travel and neuroscience}

We are grateful to the commentators who extended and updated our coverage of evidence from brain research. These include Brüne \& Brüne-Cohrs, Buckner, Carr \& Viskontas, Okuda, and Schacter \& Addis. This evidence overwhelmingly confirms the strong overlap between brain areas involved in imagining the future and in recollecting the past, and hence the Janus hypothesis. Most human brain-imaging studies, such as those summarized by Buckner, Okuda, and Schacter \& Addis, suggest that the prefrontal and parietal regions play critical roles in both episodic memory and future thinking (e.g., Addis et al. 2007, Okuda et al. 2003, Szpunar et al. 2007).

Nevertheless, Carr \& Viskontas take us to task for overemphasizing the role of the frontal lobes at the expense of the medial temporal lobe, and more particularly the hippocampus. They challenge the Stuck-in-time hypothesis concerning nonhuman animals, since the role of the hippocampus in memory is widely documented in many species, including birds. In the target article, we suggest that the prefrontal cortex may provide at least part of the extra ingredients that permit mental time travel. Carr \& Viskontas cite a recent study (Raby et al. 2007) suggesting that birds are capable of planning, despite having no prefrontal cortex. We are skeptical of this finding for reasons elaborated below, but it is becoming clear in any case that the neopallium in birds has evolved many of the functions of the neocortex (Jarvis et al. 2005). Nevertheless, there can be no doubt, as Carr \& Viskontas document, that the hippocampus is indeed critical to declarative memories (e.g., de Haan et al. 2006) and probably to foresight (e.g., Hassabis et al. 2007), and we were perhaps remiss in not emphasizing it enough. In a recent essay, de Hoz and Wood (2006) have reviewed evidence that hippocampal "place" cells in rodents might code "when" as well as "where," perhaps 
providing a substrate for episodic-like memory, or the "stage" component of the Theater hypothesis. It is possible that the hippocampus plays a more complex role in humans than in other species. For example, Aggleton and Brown (1999) have proposed a model in which the hippocampus and other diencephalic structures are activated differently by semantic and episodic memory, at least in humans, although in both cases the prefrontal cortex is implicated as well (see also Maguire et al. 2001).

Another possibility, suggested by Schacter \& Addis, is that laterality may hold the key, with evidence that the right hippocampus shows greater activity during construction of future events compared to past ones (Addis et al. 2007). But again, this same evidence also implicates a medial part of the right anterior prefrontal cortex (BA10), which they suggest may be the seat of an "episodic buffer" (Baddeley 2000) that binds multimodal information. According to the HERA (hemispheric encoding retrieval asymmetry) model (Habib et al. 2003), the left prefrontal cortex (PFC) is more involved than the right PFC in episodic memory encoding, whereas the right PFC is more involved than the left PFC in episodic memory retrieval (but see Owen 2003, for a critique). So far as we know, this model has been applied only to humans, although the once-common belief that cerebral asymmetry is uniquely human has now been thoroughly refuted (Vallortigara \& Rogers 2005).

In the target article, we noted evidence that neural loops involving the cortex and basal ganglia might be involved in mental time travel, but Okuda suggests that these are more likely to be involved in procedural prospection rather than in mental time travel as we defined it. His own work, and that of others, implicates brain networks involving medial aspects of prefrontal, temporal, and parietal cortices. He and Buckner also suggest that the brain areas involved in mental time travel may also be involved in mental traveling in space, and into the minds of others, as well as in time (see also sect. R2.4). As Okuda points out, these extensions are understandable in terms of the Theater hypothesis we advanced, and it is also worth noting that time travel almost always takes us to different places.

Buckner finds that the Theater hypothesis and the Prospection systems hypothesis offer useful frameworks, but chides us for adopting a behavioral perspective rather than a neural one. He quotes with disapproval our statement that we are not making any claims about "simulation or any other mechanisms by which this may be instantiated in the brain". Our disclaimers with respect to the Theater hypothesis were partly intended to avoid any impression that we were supporting the so-called Cartesian theater so effectively debunked by Dennett (1995), but also to disavow any expertise as to precisely how the components of mental time travel might be implemented. To make no claims is not to disapprove of such claims, and elsewhere we noted that, in the absence of phenomenological evidence from nonhuman animals, we might in future be in a position to use neurophysiological evidence as a proxy - a prospect Tulving $\boldsymbol{\&}$ Kim are also optimistic about. We are grateful, especially to Buckner, Okuda, and Schacter \& Addis, for their insights as to how the components of mental time travel suggested by the Theater hypothesis might indeed be instantiated in the brain.
Brüne \& Brüne-Cohrs make the additional point that studies of dementias and other psychopathological conditions may hold important clues. They contrast Alzheimer's disease and Korsakoff's syndrome, and suggest also that aspects of schizophrenia might be understood in terms of the Theater hypothesis. Failure in any one of the components may lead to no mental time travel, or to disturbances that may indeed be, as Lorenz suggested, more informative about the physiology than studying the intact system. Double dissociations are particularly informative, and we agree that further interdisciplinary exchange is highly desirable. Clues may come from various other syndromes (e.g., autism; Suddendorf \& Corballis 1997; Williams et al. 2001) and disorders (e.g., delusions; Gerrans 2007). Our considerations, in turn, may inform different lines of research in psychopathology (as Brüne \& Brüne-Cohrs demonstrate). For example, the discussion of animal capacities is important to developing potential "animal models" for certain conditions. Putative disturbances of the capacity to travel mentally in time can only be informed by such models to the extent that there are animal parallels. If the Uniqueness hypothesis is correct, then some aspects of these disorders may be uniquely human, too. Identification of what is not shared with other species can also hold vital clues, however, as it would seem likely that these traits are related to distinct attributes of the human brain.

\section{R4. Mental time travel and developmental psychology}

Few studies have attempted to directly investigate the development of mental time travel into the past and future (e.g. Busby \& Suddendorf 2005), but there is a relatively large literature on the development of related concepts such as autobiographical memory. Autobiographical memory is generally taken to include not just episodic memory, but also semantic knowledge that one may have about one's history. Recently studies are beginning to explore the distinction (e.g., Piolino et al. 2007). We did not include a full discussion of the developmental literature bearing on mental time travel largely due to space constraints (but see Suddendorf \& Busby 2005; Suddendorf \& Corballis 1997), but we do believe that developmental studies of mental time travel are important and that they may offer vital clues to the other relevant disciplines, too (Atance \& Meltzoff; Shettleworth).

Nelson is one of the key figures in this field, and we fully agree with her, and other prominent contributors to this literature that social and cultural factors play an important role in the development of episodic memory (e.g., Fivush et al. 2006). This literature has documented how young children first begin to refer to small parts of past events and, gradually, with the help of their caregivers, turn them into full narratives. Through the social interactions with adults and the scaffolding provided by talk about past events, children slowly seem to acquire the narrative skill required to construct their autobiographies and a more mature sense of self and other (e.g., Nelson \& Fivush 2004; Reese 2002). Although we have no doubt these social processes play an important role in determining inter- and intra-individual differences in children's abilities, we caution that these factors are not necessarily the sole determinants of mental time travel. 
One of the key lines of evidence for the importance of sociocultural effects comes from the analysis of motherchild conversations. Many studies have shown that children of mothers who elaborate more in their conversations about past events score higher on measures of autobiographical memory (for a review, see Fivush et al. 2006). There is also some, though much more limited, evidence of a similar link between elaboration and children's thinking about the future (Hudson 2006). But there can be various reasons for such correlations. For example, high-elaborating mothers may have passed on the genes that are responsible for high elaboration to their offspring. However, some data, such as differential effects of the timing of adult-child conversations on memory (e.g., McGuigan \& Salmon 2004), cannot be explained in that way and suggest that the conversations themselves do influence children's memory. Nonetheless, increased parental elaboration may not so much change or cause mental time travel per se, but may teach and encourage their reporting. In terms of the Theater hypothesis, we are dealing here entirely with the role of the broadcaster. Learning how to broadcast and understanding others' broadcasts are no doubt important. But that does not mean that it induces the other abilities we propose are involved in mental time travel. If it did, then elaborate conversations should offer a cure for people who suffer from amnesia. And conversely, people deprived of sociocultural narrative interaction (e.g., the deaf-andblind Helen Keller) should not develop mental time travel. We argue that there is more to mental time travel than just talk.

Although the importance of adult-child conversation is a common theme in the literature, most developmental theorists subscribe to something like the Components hypothesis and argue that a range of emerging capacities such as language, self-concept, and theory of mind are related to the development of autobiographic memory (e.g., Howe \& Courage 1997; Levine 2004; Nelson \& Fivush 2004; Perner 2000; Reese 2002; Suddendorf \& Corballis 1997; Welch-Ross 1995). The relation between these abilities may be multidimensional and involve other factors (e.g. such as in the Theory-of-mind hypothesis). In Suddendorf \& Corballis (1997), we note, for example, that the self in the present may be a prerequisite of mental time travel, whereas a temporally extended sense of self may be its consequence. The Theater hypothesis adds to the list of implicated skills, and we encourage developmental psychologists to study these components in addition to direct measures of mental time travel.

Little is yet known about the development of children's thinking about the future (for reviews, see Haith et al. 1994; Moore \& Lemon 2001), offering fertile ground for future research (Atance \& Meltzoff). Perhaps children's mental time travel is initially more influenced by talk about the past than the future, as Nelson claims, and the Futurefirst hypothesis may hold only for evolution rather than for development. However, key developments in foresight appear to also emerge over the preschool years (e.g., Atance \& O’Neill 2005; Suddendorf \& Busby 2005), and when asked to tell something they did do yesterday and something they will do tomorrow, 3- to 5-year-old children perform similarly on both sets of questions (Busby \& Suddendorf 2005).

One area that has been studied more extensively is children's ability to differentiate between the times of events.
Friedman reviews some of his pioneering work on this development and highlights the diverse range of mechanisms humans use in determining the times of past and future events (see Friedman 2005, for a review). Children develop these skills gradually from about age 4 onwards.

Bischof-Köhler \& Bischof draw attention to a largescale German study in which children were tested on a set of innovative measures aimed at assessing mental time travel (Bischof-Köhler 2000). It remains a bit unclear what role some of the measures, such as judging which of two egg timers finishes first, play in the capacity under discussion. However, the author found associations between these tasks and standard measures of theory of mind, substantiating the Theory-of-mind hypothesis. Furthermore, they maintain the hypothesis that we named after them (Bischof-Köhler hypothesis): that nonhuman animals cannot anticipate future need states they do not directly experience (Suddendorf \& Corballis 1997). Experimental designs have been proposed to assess future need anticipation, as future-directed action could demonstrate nonverbal capacities (Suddendorf 1994; Suddendorf \& Busby 2005; Tulving 2005). Atance \& Meltzoff warn, however, that as developmental psychologists we should not focus too much on this specific ability as the litmus test of mental time travel. We agree that the full spectrum of the emerging ability deserves study, including futureneed anticipation. Very little is yet known about how children come to develop foresight, and we suspect that developments continue well into adolescence.

\section{R5. Mental time travel and comparative psychology}

Unsurprisingly, several commentators question the claim that nonhuman animals cannot travel mentally in time (the Stuck-in-time hypothesis) and cite new research that has been published since completion of the target article in support (Bar; Carr \& Viskontas; Hurford et al.; Osvath \& Gärdenfors; Raby, Alexis, Dickinson, \& Clayton (Raby et al.); Shettleworth; Sterck \& Dufour). Although we remain skeptical about some of these conclusions as outlined here next, we commend this innovative work and, unlike Shettleworth, want to encourage more of it.

\section{R5.1. Are behavioral tests possible?}

Raby et al. lament that we did not articulate our hypotheses in specific behavioral terms, and complain that we rely too heavily on phenomenology. They also argue that phenomenology may be the only difference between episodic and semantic memory. We do not think that this is the only difference. Clearly, loss of episodic memory in amnesic patients is not merely the loss of a phenomenological sensation, or quale, but has fundamental consequences for the sufferer. In any case, the charge that we are relying too heavily on phenomenology is misleading. We took pains here and elsewhere (Suddendorf \& Busby $2003 \mathrm{~b} ; 2005)$ to move beyond first-person experience and towards objective indicators. Indeed, we pointed out that natural selection needs something to get its teeth into, and an internal feel alone is inadequate for that. We 
suggest that future-directed action is the key towards mapping animals' competence and understanding the evolution of mental time travel.

Of course, what Raby et al. want are predictions that are instantly translatable into tests with nonhuman species, such as scrub jays. Clayton and colleagues (e.g., Clayton et al. 2003; Dally et al. 2006b; Raby et al. 2007) have been prolific in developing clever ways of turning mental time travel questions into experiments involving jays caching and retrieving food. The components we discuss in the Theater hypothesis offer several new avenues, but Raby et al. dismiss components such as recursion and secondary representation that may not so easily be translated into this caching context. Yet, these are behavioral criteria that lend themselves to comparative inquiry. Suddendorf and Whiten (2001), for example, have shown that there is convincing evidence for secondary representation (the stage component) in great apes, and we see no reason why scrub jays could not be tested similarly. We thus reject the charge that we do not provide enough relevant behavioral criteria and are happy to suggest more.

Raby et al. further charge that we did not adequately explain why we advocate a focus on non-species-typical behavior in attempts to demonstrate mental time travel in animals. We made the case that the key adaptive advantage of mental time travel is the flexibility it offers for acting now to increase future survival and reproduction chances (the Flexibility hypothesis), and note that the human capacity is not restricted in content in any apparent way, but instead is open-ended and domain general. We thus argued that comparative research should focus on non-species-typical behavior because it (a) provides evidence for such flexibility and (b) rules out any role of non-cognitive behavioral predispositions governing future-directed behavior (as appears to be the case, for example, in hibernators). Scrub jays, like various other bird species, develop caching in predictable stages that suggest a prominent role of innate factors. For example, they have an early tendency to place food, as well as many other objects, without recovering these caches. Raby et al. counter that human children develop mental time travel also in a predictable fashion. However, it is not yet clear whether or not this is so. It has certainly been argued that this development is not uniform, but that there are individual and cultural differences in the way children first acquire mental time travel (see Nelson). More developmental research is required to settle this, and we maintain that studies with nonhuman animals (as well as with children) are best advised to employ novel contexts to ascertain that the behavior of interest is the result of an individual's faculty for mental time travel.

\section{R5.2. New data on animal foresight}

As we acknowledged in the target article, the BischofKöhler hypothesis cannot be true in the superficial sense that food caching is directed toward future gratification. Many species secure future needs, whether in food caching, nest building, or migration. These behaviors, though, are largely instinctive, and the hypothesis may still hold for "individual, flexible situations involving noninstinctive behaviors" (sect. 3.3 of the target article).
Several commentators (Bar; Carr \& Viskontas; Hurford et al.; Osvath \& Gärdenfors; Raby et al.; Schacter \& Addis; Sterck \& Dufour) refer to the study by Raby et al. (2007) as evidence that caching by scrub jays does display sufficient flexibility to refute the Bischof-Köhler hypothesis. In this study, eight scrub jays received breakfast in one cage and no breakfast in another cage, on alternating days. When later given the opportunity to store food while currently satiated, they cached more in the place where no breakfast was to be expected. In a second experiment, they were given different kinds of food in different locations, and later they cached more food of each particular kind in the location not associated with that food. These were taken to be clear cases in which a nonhuman species, currently satiated, cached food in anticipation of a future state of hunger or food preference. An alternative, perhaps, is that the birds based the caching, not on anticipated hunger, but on a general heuristic to balance food sources, based on previous experience of imbalance.

A closer look at the procedure raises some doubt about whether the birds were in fact acting with specific future hunger in mind. Because of counterbalancing, half the jays had received breakfast the morning of the test and the other half had not. Thus, four of the birds had reason to expect no breakfast the next day, whereas the other four should have expected to receive breakfast. Yet, the results for these two groups were combined, revealing only that, overall, the jays were caching more in the cage that was associated with no breakfast. To conclude that the jays were planning for the next morning rather than having acquired a more general rule, these two conditions would need to be compared rather than amalgamated.

Nevertheless this experiment does show flexibility beyond that expected of a purely instinctive behavior. If jays can be shown to act for specific future events, it will be interesting to see how far into the future they can "plan" and if they can also discriminate between multiple future events. In all this, however, there remains also the nagging suspicion that the flexible behavior associated with caching in scrub jays is restricted to the particular speciality of food storage. We agree with Shettleworth (2007) that it remains to be seen whether this ability is available to jays in other contexts. As we noted previously, if the same animals pass different versions of such tasks (involving different contexts and needs), then this evidence for flexibility would make the Bischof-Köhler hypothesis increasingly untenable (Suddendorf \& Busby 2005).

Some of the same commentators (Bar; Hurford et al.; Osvath \& Gärdenfors; Sterck \& Dufour) also referred to a study by Mulcahy and Call (2006), who claimed to show that apes save tools for future use, thereby refuting the Bischof-Köhler hypothesis. The limitations of this study were addressed in the target article, as well as by Suddendorf (2006), and Hurford et al. praise our assessment. Sterck \& Dufour, however, misunderstood our main critique to be that tool selection was based on current needs because the apparatus was visible, and report that in their own unpublished work, chimpanzees managed to select the right tool even when the apparatus was out of sight. But we already noted in the target article that Mulcahy and Call's "third experiment showed that the 
apes could pass the task even when they could not see the apparatus during tool selection". The issue of need in this context is not about seeing the apparatus, but about the internal motivational state (see also Bischof-Köhler \& Bischof), and we merely observed that these were neither controlled nor manipulated (and that it is likely that the apes desired grapes throughout).

Osvath \& Gärdenfors point to another recent test of the Bischof-Köhler hypothesis in which Naqshbandi and Roberts (2006) manipulated the supply of drinking water available to their subjects. They found that over repeated trials two squirrel monkeys (but not rats) gradually reversed their natural preference for four pieces of dates over one piece, when choosing four pieces resulted in provision of water after a long delay ( 3 hours), and choosing one piece was followed by water supply after a short delay (1/2 hour). Eating dates induces thirst, and subsequently not having access to something to drink for a while causes discomfort. Thus, one explanation for the gradual reversal of choice from four pieces of dates to one piece would be that the monkeys took their future thirst into account (i.e., evidence against the BischofKöhler hypothesis). As Shettleworth (2007) noted, however, the repeated exposure to the conditions means that associative learning could possibly explain the results. We would add that gradual change in preference from four pieces of dates to one piece would not necessarily be the behavior we would predict from mental time travel. If the monkeys did think ahead about the best outcome, why did they not continue to select four date pieces and simply choose to eat one or two and keep the others until enough water was available?

Eacott \& Easton report on clever experiments with rats in which they compare behavioral measures thought to tap recall with those based on familiarity. Fornixdissected rats fail on the "recall" task, yet still succeed on a "recognition" task. This innovative approach is a nice illustration of a comparative neuroscientific approach. However, the distinction between recall and recognition is not equivalent to the distinction between episodic and semantic memory (e.g., Wheeler et al. 1997). We can recall that Wellington is the capital of New Zealand, but that is because we know it, not because we can remember being told it. Thus, although the task may tap an important difference between procedural and declarative memory, no mental time travel may be involved. Nonetheless, comparing the neuronal implementation of analog behavior such as that between rats and humans, as Buckner submits, may hold vital information to clarifying the component processes of the Theater hypothesis (cf. our response to Brüne \& Brüne-Cohrs in sect. R3).

\section{R5.3. Continuity in animal competence}

Hurford et al., in spite of agreeing that there is a large gulf between animal and human mental time travel, are not convinced that animals lack it entirely. They point to an anecdote from Kanzi and to the studies with the gorilla King, which they interpret appropriately cautiously. We are of course also not convinced and acknowledge that we cannot prove the Stuck-in-time hypothesis. We believe that there is something unique about human mental time travel (the Uniqueness hypothesis) and suggest that only extensive further study can determine what, if anything, that might be. The Traveler hypothesis and the Bischof-Köhler hypothesis are two proposals. In the target article we put forward the theater metaphor and suggest that various deficits in the components we discuss could limit mental time travel (Multiple limits hypothesis). For example, foresight may be severely constraint without a capacity for recursive thought to generate content (Corballis 2007; Suddendorf \& Corballis 1997).

Bar suggests that we should consider foresight as a continuum, with nonhuman animals having as much foresight as their environment and life require, and since animal lives are less complex, they need less foresight than humans. In response, we note first that the causality may well be the other way around: Mental time travel makes human lives more complex than those of animals. Second, the idea that foresight is based on a continuum runs counter to the case we made for the Prospection systems hypothesis. Bar's notion of foresight is equivalent to what we called "future-directed capacities." However, there are reasons to distinguish between mental time travel and the various other kinds of future-directed capacities, such as instinctual, procedural, and semantic prospection. It may be possible to make a case for a unitary model of foresight, just as some scholars have done for a unitary model of memory. But such models have to explain why there are dissociations between different kinds of prospection and how they come about. For example, why does an amnesic patient, such as D.B. (Klein et al. 2002b), have selective impairment of mental time travel, but little problem providing semantic information about past and future?

Nonetheless, a case for quantitative differences in mental time travel could still be made (e.g., Carr \& Viskontas; Hurford et al.). In fact, some of the proposals we discuss could be regarded as setting quantitative limits. The Bischof-Köhler hypothesis, for example, suggests a limit to the extent of foresight (i.e., anticipation of future needs). The difference between being and not being limited in such a way could of course amount to a qualitative shift - without future need anticipation, there is little point in considering any genuinely remote future events. The Multiple limits hypothesis suggests that species might be limited by a variety of other deficits that may have both quantitative and qualitative effects.

The clever experiments we discussed in the previous section suggest that nonhuman animals may have some competence, and future research may well whittle away at some suggested limits. There are perhaps two questions here (see also sect. R5.1). One is whether such phenomena remain tied to narrow specialties, such as food-caching in birds, or whether they can cover the broad range of contexts that characterizes our own ability to travel forward and backwards in time. The second is whether examples from nonhuman animals can display the generativity of our own ability to conjure imagined events. Bar suggests that even human mental time travel may reduce to associations, but this again neglects the inventive component of human imagination, just as animal communication fails to display the generativity of human language.

Sterck \& Dufour provide further evidence about future-oriented capacities in chimpanzees, who can learn to hold a desired cookie for up to 8 minutes in order to 
exchange it for a larger one. Research with children (e.g., Mischel \& Mischel 1983) suggests that they can delay gratification for longer when the salience of the reward is decreased (e.g., when looking at a photo of the reward rather than at the actual reward), suggesting that the extent of the delay reflects inhibition or executive control, rather than the capacity to travel mentally to the future event per se. Still, this is a fairly impressive performance for chimpanzees compared to other animals. But these data do not challenge the Bischof-Köhler hypothesis, and it remains unclear how such relatively brief delay of gratification relates to the flexible capacity of humans to travel mentally to any future scenarios, be they a year or only a few minutes away. We agree of course with Sterck \& Dufour that we should not prejudge animal competence, and we acknowledged that "it remains to be established what exactly the nature and extent of modern apes' foresight (and that of other animals) is" (target article, sect. 3.3, para. 7). We also stated that "a substantial body of comparative data is required before it can be concluded that any trait is uniquely human" (target article, sect. 3.3, para. 6).

We do, however, need to start with the null hypothesis that nonhuman animals do not have this faculty or the necessary components, and we encourage researchers to attempt to falsify these statements one by one. We think it remains important, though, to stay cautious and not be too quickly impressed by apparent positive results. We recommend keeping Occam's razor sharp. Note that negative findings are seldom published, and hence Osvath \& Gärdenfors' observation that most studies have reported positive results for animal foresight may not be surprising. There are, of course, good epistemological reasons not to emphasize failures to reject the null hypothesis, but we might be missing out on instructive information about the systematic failures of nonhuman animals. We would therefore like to encourage comparative researchers to also investigate and report the limits of their subjects' capacities.

\section{R5.4. The future of animal studies of mental time travel}

In stark contrast to our call for more studies, Shettleworth thinks this is all heading in the wrong direction and suggests a moratorium on animal studies of mental time travel (at least until developmental psychology has made some progress). Shettleworth thinks our questions are flawed because we can never know what is on an animal's mind, and because it moves the field in non-productive directions. The first charge is easily dispelled. We can also never know for certain what even another human's mental experience is like (language or not), but that does not stop us studying the mind (though it did during the behaviorist era). With respect to the second charge, quite contrary to Shettleworth's assertion, recent work on mental time travel in animals has been very productive indeed (in addition to our review, see also Dere et al. 2006; Roberts 2006; Zentall 2006). In fact, Shettleworth (2007) herself proceeds to demonstrate this when discussing Raby et al.'s (2007) recent study. If these researchers had followed her suggestion, that paper would not exist. As Raby et al. acknowledge at the beginning of their commentary, our analyses have provided a theoretical framework for their work. Whether one agrees or disagrees with our proposals and interpretations, there can be little argument that the questions we have raised have sparked very productive research programs.

We agree with Shettleworth that questions like "what components of process $\mathrm{X}$ are and are not shared among what species and why, in evolutionary, functional, and perhaps neurological terms?" are productive, and indeed our target article raised many more questions than the Stuck-in-time hypothesis (e.g., Table 1). Since humans can travel mentally in time, the natural question of whether nonhuman animals can do so, too, can inspire the pursuit of more detailed analyses of animal capacities. Raising such big questions about consciousness and its evolution can be very useful. Consider the case of theory of mind, a field instigated by Premack and Woodruff's (1978) seminal Behavioral and Brain Sciences article in which they searched for an existence proof. The article sparked a flurry of comparative research that has led to real progress. In the process, it kick-started perhaps one of the most productive research areas in developmental psychology and galvanized research in neuroscience, philosophy, and other disciplines. Would it be more productive to retreat to questions that dare not mention mind or consciousness for fear of a return to anthropomorphism?

Of course, we advocate that comparative psychologists be cautious with their interpretation - after all, we have been promoting such caution in regard to claims about mental time travel. But that does not mean we would want to abandon these efforts. There are various reasons why such questions are popular, as Shettleworth (2007) herself noted: "These include Donald Griffin's exhortations to ethologists to tackle animal consciousness, legitimisation of the study of consciousness in the cognitive sciences generally, and the prospect of understanding the neural and genetic bases of conscious processes using 'animal models"' (p. 826) Whereas the first point merely offers a glimpse at the author's position, the latter two are very compelling reasons to pursue these research questions, we think. We may learn a lot about cognition and the brain in the process (see Buckner; Carr \& Viskontas; Eacott \& Easton; Hurford et al.; Tulving \& Kim).

Another good reason to study what is unique about the human mind, and what is not, is that this can in various ways inform us about the evolution of the human mind (Suddendorf 2004). In this context, convergent evolution of some capacities (as perhaps in jays) may be less relevant than the potentially homologous abilities of our closest living primate relatives.

\section{R6. Mental time travel and evolutionary psychology}

The Prime mover hypothesis has not attracted much controversy. Several commentators acknowledge that humans have an extraordinary capacity for foresight (e.g., Ainslie; Bischof-Köhler \& Bischof; Buckner; Osvath \& Gärdenfors) whether or not they argued for some abilities in other primates. We certainly think that foresight has played a major role in human survival, providing us with unparalleled flexibility in adaptive and proactive behavior (the Flexibility hypothesis). Consider, for example, the long history of humans carrying with them 
diverse sets of tools in preparation for future happenings (e.g., Suddendorf 2006). The capacity for mental time travel would on the face of it appear to be necessary for the emergence of civilizations. Yet, as Mesoudi points out, it still needs to be established how specifically, and to what extent, mental time travel has in fact influenced the evolution of human culture.

Some versions of Darwinian cultural evolution suggest that cultural change is just as blind as biological change. Thus, the very existence of mental time travel can be seen as a potential threat to the notion of Darwinian cultural evolution, as Mesoudi considers so thoughtfully. We agree that the evidence we present makes simple denial that humans have foresight an untenable escape. We also agree that this does not imply that Darwinian cultural evolution is a lost cause. As Mesoudi et al. (2006) have shown, there are many parallels between cultural and biological evolution that are worth considering, even if there are also important differences. In modern history there are certainly cases of explicit social engineering where, say, Marxism was implemented based on plans for future change. Although this demonstrates that cultural change can be induced by foresight, the failures of these attempts also illustrate that foresight is not fortune telling. Just as Mesoudi makes a distinction between the capacity for culture and the content of culture, then, we need to distinguish the capacity for mental time travel and its content (cf. Tulving \& Kim). We suspect that many aspects of human culture are not top-down, but bottom-up related to our shared ability to reconstruct past and imagine future events. Discussion of these effects goes beyond the scope of the current treatment, but we would like to support Mesoudi's call for researchers to investigate how mental time travel might influence cultural change.

In turn, of course, culture and language may also influence the way people travel mentally in time, as Nelson points out. For example, cultures may determine what it is that is important to remember or to anticipate. Some cultures may value the future more than the past, or vice versa, and some may emphasize the here-and-now (Nelson \& Fivush 2004). For example, in one study Chinese autobiographical memories were more social than those of U.S. participants who recalled more events oriented towards the self (Conway et al. 2005). Nonetheless, the reminiscence bump was evident across the cultural groups tested. Mental time travel seems to be a cross-cultural universal (cf. Tulving \& Kim). People of all cultures, with the possible but controversial exception of an Amazonian tribe, the Piraha (Everett 2005), can plan for next week and can remember what happened last week.

Osvath \& Gärdenfors prudently advise that one should be agnostic about whether animals have some mental time travel capacity or not. They nonetheless suspect that primates may have some proficiency in the social realm. Although we agree that primate social intelligence is very impressive, we do not think it is "almost equally developed" as that of humans (e.g., consider the scale of human cooperation). Nonetheless, we agree with Osvath \& Gärdenfors that social factors alone cannot explain why humans but not other primates may have evolved mental time travel. In fact, we pointed to this problem for social accounts of the evolution of human intelligence more generally when we wrote: "One problem for such an account is to explain why the cognitive arms race seems to have persisted longer in humans than in other primates, resulting in apparently unique cognitive skills, including, perhaps, mental time travel" and we pointed to a way out of the conundrum:

A potential explanation, suggested by Alexander and colleagues (Alexander 1989; Flinn et al. 2005), is that once early hominins obtained a certain level of "ecological dominance," ... they were faced with increased competition from their own species.

This "ecological dominance" may have led our ancestors down a different path of intergroup competition, resulting in the emergence of language, theory of mind, mental time travel, and so forth.

Note, however, that we did not really advocate the socialintelligence account, even in this modified form. Instead we discussed two alternative (and potentially complementary) accounts, one based on fire and one on throwing. Indeed, we refer readers to Osvath and Gärdenfors' (2005) own excellent analysis in this context. As explicitly stated in the target article, however, we remain agnostic about which, if any, of these accounts is correct, and instead discuss them to show that there are viable explanations that do not demand that we ascribe higher mental capacity to modern apes, and to our common ancestor with apes, than is currently accepted.

We suggest that mental time travel evolved in the genus Homo (the Lower Paleolithic emergence hypothesis). The archaeological record indicates to us that by 1.5 million years ago our ancestors already had some mental time travel ability. Osvath \& Gärdenfors agree that "advanced" mental time travel was evident then and provide reasons to suggest that some capacity was already present in the Oldowan culture. Though the details of its effect still need be established, as Mesoudi warns, we maintain that only with the capacity for mental time travel could human cultures have invented such fundamental characteristics of civilization as trade, law, and religion.

\section{R7. Conclusion}

In conclusion, the commentaries signal an increased multidisciplinary interest in mental time travel. We hope this treatment will galvanize these fledging developments, regardless of whether or not our specific proposals stand the test of time.

\section{References}

[The letters " $a$ " and " $r$ " before author's initials stand for target article and response references, respectively.]

Addis, D. R., Wong, A. T. \& Schacter, D. L. (2007) Remembering the past and imagining the future: Common and distinct neural substrates during event construction and elaboration. Neuropsychologia 45(7):1363-77. [RLB, VAC, JO, DLS, rTS, ET]

(in press) Age-related changes in the episodic simulation of future events. Psychological Science. [DLS]

Aggleton, J. P. \& Brown, M. W. (1999) Episodic memory, amnesia and the hippocampal-anterior thalamic axis. Behavioral and Brain Sciences 22(3):425-89. [rTS] 
Ainslie, G. (2001) Breakdown of will. Cambridge University Press. [GA] (2006) Motivation must be momentary. In: Understanding choice, explaining behaviour, ed. J. Elster, O. Gjelsvik, A. Hylland \& K. Moene, pp. 9-24. Unipub Forlag. [GA]

Albrecht, U. \& Eichele, G. (2003) The mammalian circadian clock. Current Opinion in Genetics and Development 13(3):271-77. [aTS]

Alexander, R. D. (1989) Evolution of the human psyche. In: The human revolution: Behavioral and biological perspectives on the origins of modern humans, ed. P. Mellars \& C. Stringer, pp. 455-531. Princeton University Press. [arTS

Allen, C. (2002) A skeptic’s progress. Biology and Philosophy 17(5):695-702. [aTS]

Anderson, J. R. \& Schooler, L. J. (1991) Reflections of the environment in memory. Psychological Science 2:396-408. [aTS]

Atance, C. M. \& Meltzoff, A. N. (2005) My future self: Young children's ability to anticipate and explain future states. Cognitive Development 20:34161. [CMA, aTS]

(2006) Preschoolers' current desires warp their choices for the future. Psychological Science 17(7):583-87. [CMA, aTS]

Atance, C. M. \& O'Neill, D. K. (2005) The emergence of episodic future thinking in humans. Learning and Motivation 36(2):126-44. [arTS]

Aunger, R., ed. (2000) Darwinizing culture. Oxford University Press. [AM]

Babb, S. J. \& Crystal, J. D. (2005) Discrimination of what, when, and where: Implications for episodic-like memory in rats. Learning and Motivation 36:177-89. [aTS]

(2006) Episodic-like memory in the rat. Current Biology 16:1317-21. [MBa]

Baddeley, A. D. (1992) Working memory. Science 255:556-59. [aTS]

(2000) The episodic buffer: A new component of working memory? Trends in Cognitive Sciences 4(11):417-23. [DLS, rTS]

Bar, M. (2004) Visual objects in context. Nature Reviews Neuroscience 5:61729. $[\mathrm{MBa}]$

(2007) The proactive brain: Using analogies and associations to generate predictions. Trends in Cognitive Sciences 11(7):280-89. [MBa]

Bar, M., Aminoff, E., Mason, M. \& Fenske, M. (2007) The units of thought. Hippocampus 17(6):420-28. [MBa]

Bard, K., Todd, B. K., Bernier, C., Love, J. \& Leavens, D. A. (2006) Self-awareness in human and chimpanzee infants: What is measured and what is meant by the mark and mirror test. Infancy 9:191-219. [aTS]

Barrett, L., Henzi, P. \& Dunbar, R. (2003) Primate cognition: From “what now?" to "what if?" Trends in Cognitive Sciences 7(11):494-97. [EHMS, aTS]

Bartlett, F. C. (1932) Remembering: A study in experimental and social psychology Cambridge University Press. [rTS]

Basalla, G. (1988) The evolution of technology. Cambridge University Press. [AM]

Bateson, P. (2004) Evolution and learning: The Baldwin effect reconsidered. Biology and Philosophy 19(2):283-98. [aTS]

Baumeister, R. F. (1998) The self. In: Handbook of social psychology, vol. 1, 4th edition, ed. D. T. Gilbert, S. T. Fiske \& G. Lindzey, pp. 680-740. McGrawHill. $[\mathrm{AD}]$

Bechara, A. \& Damasio, A. R. (2005) The somatic marker hypothesis: A neural theory of economic decision. Games and Economic Behavior 52:33672. $[\mathrm{AD}]$

Benton, T. (2000) Social causes and natural relations. In: Alas, poor Darwin, ed. H. Rose \& S. Rose, pp. 206-24. Harmony. [AM]

Berridge, K. C. \& Robinson, T. (1998) What is the role of dopamine in reward: Hedonic impact, reward learning, or incentive salience: Brain Research Reviews 28:309-69. [GA]

Bickerton, D. (2002) Foraging versus social intelligence in the evolution of protolanguage. In: The transition to language, ed. A. Wray, pp. 207-25. Oxford University Press. [MO]

Bischof, N. (1978) On the phylogeny of human morality. In: Morality as biological phenomenon, ed. G. S. Stent, pp. 53-74. Dahlem Konferenzen. [rTS] (1985) Das Rätzel Ödipus [The Oedipus riddle]. Piper. [aTS]

Bischof-Köhler, D. (1985) Zur Phylogenese menschlicher Motivation [On the phylogeny of human motivation]. In: Emotion und Reflexivität, ed. L. H. Eckensberger \& E. D. Lantermann, pp. 3-47. Urban \& Schwarzenberg. [arTS]

(2000) Kinder auf Zeitreise [Children on time travel. Theory of mind, time comprehension, and action control]. Huber. [DB-K, rTS]

Boaz, N. T., Ciochon, R. L., Xu, Q. \& Liu, J. (2004) Mapping and taphonomic analysis of the Homo erectus loci at Locality 1 Zhoukoudian, China. Journal of Human Evolution 46(5):519-49. Available at: http://www.sciencedirect.com/science/ article/B6WJS-4C5PTXB-1/2/4378ec5leb16b19e76acf42ee97fb6el [aTS]

Boyd, R. \& Richerson, P. J. (1985) Culture and the evolutionary process. University of Chicago Press. [AM]

Brown, R. \& Kulik J. (1977) Flashbulb memories. Cognition 5:73-99. [J-LD] Browning, R. (1896) The poetical works. Smith, Elder \& Co. [aTS]

Brüne, M. (2006) The evolutionary psychology of obsessive-compulsive disorder: The role of cognitive metarepresentation. Perspectives in Biology and Medicine 49(3):317-29. [MBr, rTS]
Brüne, M. \& Brüne-Cohrs, U. (2006) "Theory of mind" - Evolution, ontogeny, brain mechanisms and psychopathology. Neuroscience and Biobehavioral Reviews 30:437-55. [MBr]

Bryant, J. M. (2004) An evolutionary social science? A skeptic's brief, theoretical and substantive. Philosophy of the Social Sciences 34:451-92. [AM]

Buckner, R. L. \& Carroll, D. C. (2007) Self-projection and the brain. Trends in Cognitive Sciences 11(2):49-57. [RLB, JO, DLS, rTS, ET]

Bugnyar, T. \& Heinrich, B. (2006) Pilfering ravens, Corvus corax, adjust their behavior to social context and identity of competitors. Animal Cognition 9:369-76. [aTS]

Bunsey, M. \& Eichenbaum, H. (1995) Selective damage to the hippocampal region blocks long-term retention of a natural and nonspatial stimulus-stimulus association. Hippocampus 5:546-56. [VAC]

Burgess, P. W., Gilbert, S. J., Dumontheil, I. \& Simons, J. S. (2005) The gateway hypothesis of rostral prefrontal cortex (area 10) function. In: Speed, control, and age: In honour of Patrick Rabbitt, ed. J. Duncan, L. Phillips \& P. MacLeod, pp. 217-48. Oxford University Press. [JO]

Burgess, P. W., Gilbert, S. J., Okuda, J. \& Simons, J. S. (2006) Rostral prefrontal brain regions (area 10): A gateway between inner thought and the external world? In: Disorders of volition, ed. W. Prinz \& N. Sebanz, pp. 373-95. MIT Press. [JO]

Burke, D. \& Fulham, B. J. (2003) An evolved spatial memory bias in a nectarfeeding bird. Animal Behavior 66:695-701. [aTS]

Busby, J. \& Suddendorf, T. (2005) Recalling yesterday and predicting tomorrow. Cognitive Development 20(3):362-72. [arTS]

Call, J. (2001) Chimpanzee social cognition. Trends in Cognitive Sciences 5:38893. [aTS]

(2004) Inferences about the location of food in the great apes (Pan pansicus, Pan troglodytes, Gorilla gorilla, and Pongo pygmaeus). Journal of Comparative Psychology 118:232-41. [aTS]

(2005) The self and other: A missing link in comparative social cognition. In: The missing link in cognition, ed. H. S. Terrace \& J. Metcalfe, pp. 321-41. Oxford University Press. [aTS]

Call, J. \& Carpenter, M. (2001) Do chimpanzees and children know what they have seen? Animal Cognition 4:207-20. [aTS]

Call, J., Hare, B. \& Tomasello, M. (1998) Chimpanzee gaze following in an object choice task. Animal Cognition 1:89-99. [aTS]

Calvin, W. H. (1982) Did throwing stones shape hominid brain evolution? Ethology and Sociobiology 3(3):115-24. [aTS]

Campbell, D. T. (1960) Blind variation and selective retentions in creative thought as in other knowledge processes. Psychological Review 67:380-400. [AM]

Carneiro, R. L. (1985) Comment on: "Darwinian selection, symbolic variation, and the evolution of culture" by D. Rindos. Current Anthropology 26:7778. $[\mathrm{AM}]$

Chater, N. (2005) Mendelian and Darwinian views of memes and cultural change. In: Perspectives on imitation, vol. 2, ed. S. Hurley \& N. Chater, pp. 355-62. MIT Press. [AM]

Clayton, N. S., Bussey, T. J. \& Dickinson, A. (2003) Can animals recall the past and plan for the future? Nature Reviews Neuroscience 4:685-91. [CR, aTS]

Clayton, N. S., Bussey, T. J., Emery, N. J. \& Dickinson, A. (2003) Prometheus to Proust: The case for behavioural criteria for "mental time travel". Trends in Cognitive Sciences 7(10):436-37. [rTS]

Clayton, N. S. \& Dickinson, A. (1998) Episodic-like memory during cache recovery by scrub jays. Nature $395: 272-78$. [aTS]

Clayton, N. S., Yu, K. S. \& Dickinson, A. (2001) Scrub Jays (Aphelocoma coerulescens) form integrated memories of the multiple features of caching episodes. Journal of Experimental Psychology: Animal Behavior Processes 27:1729. [aTS]

Collier-Baker, E. \& Suddendorf, T. (2006) Do chimpanzees (Pan troglodytes) and 2-year-old children (Homo sapiens) understand double invisible displacement? Journal of Comparative Psychology 120:89-97. [aTS]

Conway, M. A. (2005) Memory and the self. Journal of Memory and Language 53:594-628. [AD]

Conway, M. A., Meares, K. \& Standart, S. (2004) Images and goals. Memory $12: 525-31 . \quad[\mathrm{AD}]$

Conway, M. A., Wang, Q., Hanyu, K. \& Haque, S. (2005) A cross-cultural investigation of autobiographical memory - On the universality and cultural variation of the reminiscence bump. Journal of Cross-Cultural Psychology 36(6):739_ 49. $[\mathrm{rTS}]$

Conway, M., Singer, J. A. \& Tagini, A. (2004) The self and autobiographical memory: Correspondence and coherence. Social Cognition 22:491-529. [aTS]

Cook, R., Brown, M. \& Riley, D. (1983) Flexible memory processing by rats: Use of prospective and retrospective information. Journal of Experimental Psychology: Animal Behavior Processes 11:453-69. [JRH]

Corballis, M. C. (1991) The lopsided ape. Oxford University Press. [aTS] (2003) Recursion as the key to the human mind. In: From mating to mentality: Evaluating evolutionary psychology, ed. K. Sterelny \& J. Fitness, pp. 155-71. Psychology Press. [aTS] 
(2004) The origins of modernity: Was autonomous speech the critical factor? Psychological Review 111:543-52. [aTS]

(2007) The uniqueness of human recursive thinking. American Scientist 95:24250. $\quad[\mathrm{rTS}]$

(in press) Language, recursion, and starlings. Cognitive Science. [aTS]

Corballis, M. C. \& Suddendorf, T. (in press) Memory, time and language. In: What makes us human?, ed. C. Pasternak, pp. 17-36. Oneworld. [arTS]

Cornuejols, A. \& Miclet, L. (2002) Apprentissage artificiel - Concepts et algorithmes [Machine Learning: Concepts and algorithms]. Eyrolles. [J-LD]

Dally, J. M., Clayton, N. S. \& Emery, N. J. (2006a) The behavior and evolution of cache protection and pilferage. Animal Behavior 72:13-23. [aTS]

Dally, J. M., Emery, N. J. \& Clayton, N. S. (2006b) Food-caching western scrub-jays keep track of who was watching when. Science 312(5780):1662-65. [MO, $\operatorname{arTS}]$

Damasio, A. R., Grabowski, T. J., Bechara, A., Damasio, H., Ponto, L. L. B., Parvizi, J. \& Hichwa, R. D. (2000) Subcortical and cortical brain activity during the feeling of self-generated emotions. Nature Neuroscience 3:1049-56. [AD]

D’Argembeau, A., Comblain, C. \& Van der Linden, M. (2003) Phenomenal characteristics of autobiographical memories for positive, negative, and neutral events. Applied Cognitive Psychology 17:281-94. [AD]

D'Argembeau, A. \& Van der Linden, M. (2004) Phenomenal characteristics associated with projecting oneself back into the past and forward into the future: Influence of valence and temporal distance. Consciousness and Cognition 13:844-58. [AD, aTS]

(2005) Influence of emotion on memory for temporal information. Emotion 5:503-07. [AD]

(2006) Individual differences in the phenomenology of mental time travel: The effect of vivid visual imagery and emotion regulation strategies. Consciousness and Cognition 15(2):342-50. [AD, rTS, ET]

Dasgupta, S. (2004) Is creativity a Darwinian process? Creativity Research Journal $16: 403-13 . \quad[\mathrm{AM}]$

Davachi, L. (2006) Item, context and relational episodic encoding in humans. Current Opinion in Neurobiology 16:693-700. [VAC]

Davidson, P. R. \& Wolpert, D. M. (2005) Widespread access to predictive models in the motor system: A short review. Journal of Neural Engineering 2:S31319. $[\mathrm{JH}]$

Dawkins, R. (1996) The blind watchmaker. W.W. Norton. [AM]

(2000) An open letter to Prince Charles. Available at http://www.edge.org/ 3rd_culture/prince/prince_index.html [aTS]

de Haan, M., Mishkin, M., Baldeweg, T. \& Vargha-Khadem, F. (2006) Human memory development and its dysfunction after early hippocampal injury. Trends in Neurosciences 29(7):374-81. [rTS]

de Hoz, L. \& Wood, E. R. (2006) Dissociating the past from the present in the activity of place cells. Hippocampus 16(9):704-15. [rTS]

de Kort, S. R., Dickinson, A. \& Clayton, N. S. (2005) Retrospective cognition by food-caching western scrub-jays. Learning and Motivation 36:159-76. [WJF]

de Waal, F. (1982) Chimpanzee politics - Power and sex among apes. Jonathan Cape. $[\mathrm{MO}]$

Deacon, T. (1997) The symbolic species: The co-evolution of language and the brain. W.W. Norton. [aTS]

Dennett, D. C. (1995) Darwin's dangerous idea. Simon \& Schuster. [arTS]

Dennett, D. \& McKay, R. (2006) A continuum of mindfulness. Behavioral and Brain Sciences 29:353-54. [AM]

Dere, E., Huston, J. P. \& Silva, M. A. S. (2005) Episodic-like memory in mice: Simultaneous assessment of object, place and temporal order memory. Brain Research Protocols 16(1-3):10-19. [aTS]

Dere, E., Kart-Teke, E., Huston, J. P. \& Silva, M. A. D. (2006) The case for episodic memory in animals. Neuroscience and Biobehavioral Reviews 30(8):120624. [rTS]

Dessalles, J.-L. (2006) Human language in the light of evolution. In: JEP 2006: Actes des XXVIes journées d'étude sur la parole, AFCP - IRISA - ISCA, pp. 17-23. Dinard. Available at: http://www.enst.fr/ jld/papiers/pap.evol/ Dessalles_06072301.pdf [J-LD]

(2007) Why we talk - The evolutionary origins of language. Oxford University Press. Abstract available at http://www.enst.fr/ jld/WWT/ [J-LD]

(in press) Complexité cognitive appliquée à la modélisation de l'intérêt narratif [Cognitive complexity as a determining factor of narrative interest]. Intellectica 45. [J-LD]

Dretske, F. I. (1982) The informational character of representation. Behavioral and Brain Sciences 5:376-77. [aTS]

Dudai, Y. \& Carruthers, M. (2005a) Memory: Some systems in the brain may be better equipped to handle the future than the past. Nature 434:567. [arTS] (2005b) The Janus face of mnemosyne. Nature 434:823-24. [DLS]

Dufour, V. \& Sterck, E. H. M. (submitted) Planning capacities in exchange and tool-use tasks in chimpanzees (Pan troglodytes). [EHMS]

Dufour, V., Pelé, M., Sterck, E. H. M. \& Thierry, B. (2007) Chimpanzee anticipation of food return: Coping with waiting time in an exchange task. Journal of Comparative Psychology 121(2):145-55. [EHMS]
Dunbar, R. I. M. (1998) Theory of mind and the evolution of language. In: Approaches to the evolution of language: Social and cognitive bases, ed. J. R. Hurford, M. Studdert-Kennedy \& C. Knight, pp. 92-110. Cambridge University Press. [J-LD]

(2003) The social brain: Mind, language, and society in evolutionary perspective. Annual Review of Anthropology 32:163-81. [aTS]

Eacott, M. J., Easton, A. \& Zinkivskay, A. (2005) Recollection in an episodiclike memory task in the rat. Learning and Memory 12(3):22123. [MJE, aTS $]$

Easton, A., Eacott, M. J., Zinkivskay, A. \& Jimenez-Rodriguez, M. (2006) Fornix lesions and episodic-like memories: Recollection impaired, familiarity intact. Society for Neuroscience Abstracts 66(5). [MJE]

Eggins, S. \& Slade, D. (1997) Analysing casual conversation. Equinox. [J-LD]

Eichenbaum, H. \& Fortin, N. J. (2005) Bridging the gap between brain and behavior: Cognitive and neural mechanisms of episodic memory. Journal of the Experimental Analalysis of Behaviour 84:619-29. [MBa]

Eichenbaum, H., Fortin, N. J., Ergorul, C., Wright, S. P. \& Agster, K. L. (2005) Episodic recollection in animals: "If it walks like a duck and quacks like a duck..." Learning and Motivation 36(2):190-207. [WJF, aTS]

Eisenberg, Z. W. (2006) From now to then: Parents and children talk about the past and the future. Unpublished doctoral dissertation, City University of New York Graduate Center, New York. [KN]

Eldridge, L. L., Engel, S. A., Zeineh, M. M., Bookheimer, S. Y. \& Knowlton, B. J. (2005) A dissociation of encoding and retrieval processes in the human hippocampus. Journal of Neuroscience 25:3280-86. [VAC]

Eldridge, L. L., Knowlton, B. J., Furmanski, C. S., Bookheimer, S. Y. \& Engel, S. A. (2000) Remembering episodes: A selective role for the hippocampus during retrieval. Nature Neuroscience 3:1149-52. [VAC]

Ellsworth, P. C. \& Scherer, K. R. (2003) Appraisal processes in emotion. In: Handbook of affective sciences, ed. R. J. Davidson, K. R. Scherer \& H. H. Goldsmith, pp. 572-95. Oxford University Press. [AD]

Emery, N. J. \& Clayton, N. S. (2001) Effects of experience and social context on prospective caching strategies by scrub jays. Nature 414:443-46. [aTS]

(2004) The mentality of crows: Convergent evolution of intelligence in corvids and apes. Science 306:1903-907. [aTS]

Epstein, J. M. \& Axtell, R. (1996) Growing artificial societies. MIT Press. [AM]

Etscorn, F. \& Stephens, R. (1973) Establishment of conditioned taste aversions with a 24-hour CS-US interval. Physiological Psychology 1:251-53. [aTS]

Everett, D. L. (2005) Cultural constraints on grammar and cognition in Piraha Another look at the design features of human language. Current Anthropology 46(4):621-46. [rTS]

Feigenson, L., Dehaene, S. \& Spelke, E. (2004) Core systems of number. Trends in Cognitive Sciences 8:307-14. [SJS]

Ferbinteanu, J. \& Shapiro, M. L. (2003) Prospective and retrospective memory coding in the hippocampus. Neuron 40:1227-39. [RLB]

Fitch, W. T. \& Hauser, M. D. (2004) Computational constraints on syntactic processing in a nonhuman primate. Science 303:377-80. [aTS]

Fivush, R., Haden, C. A. \& Reese, E. (2006) Elaborating on elaborations: Role of maternal reminiscing style in cognitive and socioemotional development. Child Development 77(6):1568-88. [rTS]

Flinn, M. V., Geary, D. C. \& Ward, C. V. (2005) Ecological dominance, social competition, and coalitionary arms races: Why humans evolved extraordinary intelligence. Evolution and Human Behavior 26:10-46. [arTS]

Fortin, N. J., Wright, S. P. \& Eichenbaum, H. (2004) Recollection-like memory retrieval in rats is dependent on the hippocampus. Nature 431:18891. [MJE]

Foster, D. J. \& Wilson, M. A. (2006) Reverse replay of behavioural sequences in hippocampal place cells during the awake state. Nature 440:680-83. [RLB]

Friedman, W. J. (1992) Children's time memory: The development of a differentiated past. Cognitive Development 7:171-87. [WJF]

(1993) Memory for the time of past events. Psychological Bulletin 113(1):4466. [MJE, WJF, aTS]

(1996) Distance and location processes in memory for the times of past events. In: The psychology of learning and motivation, vol. 35, ed. D. L. Medin, pp. 1-41. Academic Press. [WJF]

(2000) The development of children's knowledge of the times of future events. Child Development 71(4):913-32. [aTS]

(2001) Memory processes underlying humans' chronological sense of the past. In: Time and memory: Issues in philosophy and psychology, ed. C. Hoerl \& T. McCormack, pp. 139-67. Oxford University Press. [WJF]

(2003) The development of a differentiated sense of the past and the future. In: Advances in child development and behavior, vol. 31, ed. R. Kail, pp. 229-69. Academic Press. [WJF]

(2004) Time in autobiographical memory. Social Cognition 22:605-21. [WJF] (2005) Developmental and cognitive perspectives on humans' sense of the times of past and future events. Learning and Motivation 36:145-58. [arTS] 
Friedman, W. J. \& Huttenlocher, J. (1997) Memory for the times of "60 Minutes" stories and news events. Journal of Experimental Psychology: Learning, Memory, and Cognition 23:560-69. [WJF]

Friedman, W. J. \& Kemp, S. (1998) The effects of elapsed time and retrieval on young children's judgements of the temporal distances of past events. Cognitive Development 13:335-67. [aTS]

Gallup, G. G. (1970) Chimpanzees: Self recognition. Science 167:86-87. [aTS]

Garcia, J., Ervin, F. R. \& Koelling, R. (1966) Learning with prolonged delay of reinforcement. Psychonomic Science 5:121-22. [aTS]

Garcia, J. \& Koelling, R. (1966) Relation of cue to consequence in avoidance learning. Psychonomic Science 4:123-24. [aTS]

Gardiner, J. M., Ramponi, C. \& Richardson-Klavehn, A. (2002) Recognition of memory and decision processes: A meta-analysis of remember, know, and guess responses. Memory 10(2):83-98. [aTS]

Gentner, T. O., Fenn, K. M., Margoliash, D. \& Nusbaum, H. C. (2006) Recursive syntactic pattern learning by songbirds. Nature 440:1204-207. [aTS]

Gerrans, P. (2007) Mechanisms of madness: Evolutionary psychiatry without evolutionary psychology. Biology and Philosophy 22:36-56. [rTS]

Gibson, J. J. (1979) The ecological approach to visual perception. Houghton Mifflin. [JRH]

Gilbert, D. T. (2006) Stumbling on happiness. Knopf. [MBa, RLB, aTS]

Gilbert, D. T., Gill, M. J. \& Wilson, T. D. (2002) The future is now: Temporal correction in affective forecasting. Organizational Behavior and Human Decision Processes 88:430-44. [CMA, aTS]

Gilbert, S. J., Dumontheil, I., Simons, J. S., Frith, C. D. \& Burgess, P. W. (2007) Comment on "Wandering minds: The default network and stimulusindependent thought." Science 317:43. [JO]

Glymour, C. (2002) The mind's arrows: Bayes nets and graphical causal models. MIT Press. [JH]

Gopnik, A. \& Slaughter, V. (1991) Young children's understanding of changes in their mental states. Child Development 62(1):98-110. [aTS]

Habib, R., Nyberg, L. \& Tulving, E. (2003) Hemispheric asymmetries of memory: The HERA model revisited. Trends in Cognitive Sciences 7(6):241-45. [rTS]

Hailman, J. P. \& Ficken, M. S. (1986) Combinatorial animal communication with computable syntax: Chick-a-dee calling qualifies as "language" by structural linguistics. Animal Behavior 34:1899-901. [aTS]

Haith, M. M., Benson, J. B., Roberts, R. J., Jr. \& Pennington, B. F., eds. (1994) The development of future-oriented processes. University of Chicago Press. [rTS]

Haldane, J. B. S. (1927) A mathematical theory of natural and artificial selection, Part V: Selection and mutation. Proceedings of the Cambridge Philosophical Society 23:838-44. [aTS]

Hallos, J. (2005) "15 minutes of fame:" Exploring the temporal dimension of Middle Pleistocene lithic technology. Journal of Human Evolution 49:155-79. [aTS]

Hallpike, C. R. (1986) The principles of social evolution. Clarendon Press. [AM]

Hampton, R. R. (2001) Rhesus monkeys know when they remember. Proceedings of the National Academy of Sciences of the United States of America 98(9):5359-62. [aTS, SJS]

Hampton, R. R., Hampstead, B. M. \& Murray, E. A. (2005) Rhesus monkeys (Macaca mulatta) demonstrate robust memory for what and where, but not for when, in an open-field test of memory. Learning and Motivation 36:245-59. [aTS]

Hampton, R. R. \& Schwartz, B. L. (2004) Episodic memory in nonhumans: What and where, is when? Current Opinion in Neurobiology 14:192-97. [aTS]

Hampton, R. R., Zivin, A. \& Murray, E. A. (2004) Rhesus monkeys (Macaca mulatta) discriminate between knowing and not knowing and collect information as needed before acting. Animal Cognition 7:239-46. [SJS]

Hare, B., Call, J., Agnetta, B. \& Tomasello, M. (2000) Chimpanzees know what conspecifics do and do not see. Animal Behavior 59:771-85. [aTS]

Hare, B., Call, J. \& Tomasello, M. (2001) Do chimpanzees know what conspecifics know? Animal Behavior 61:139-51. [aTS]

Harner, L. (1975) Yesterday and tomorrow: Development of early understanding of the terms. Developmental Psychology 11(6):864-65. [aTS]

Hassabis, D., Kumaran, D., Vann, S. D. \& Maguire, E. A. (2007) Patients with hippocampal amnesia cannot imagine new experiences. Proceedings of the National Academy of Sciences USA 104(5):1726-31. [MBa, RLB, VAC, WJF, JO, DLS, rTS]

Hasselmo, M. E., Wyble, B. P. \& Wallenstein, G. V. (1996) Encoding and retrieva of episodic memories: Role of cholinergic and GABAergic modulation in the hippocampus. Hippocampus 6:693-708. [VAC]

Hauser, M. D. (2001) Folk physics for apes - The chimpanzee's theory of how the world works. Science 291(5503):440-41. [aTS]

(2003) Knowing about knowing: Dissociations between perception and action systems over evolution and during development. Annals of the New York Academy of Science 1001:79-103. [aTS]

Hauser, M. D., Chomsky, N. \& Fitch, W. T. (2002) The faculty of language: What is it, who has it, and how did it evolve? Science 298:1569-79. [aTS]

Hazlitt, W. (1805) An essay on the principles of human action. J. Johnson. [arTS]

Henderson, J., Hurly, T. A., Bateson, M. \& Healy, S. D. (2006) Timing in free-living rufous hummingbirds, Selasphorus rufus. Current Biology 16(5):512-15. [aTS]
Henrich, J. \& McElreath, R. (2003) The evolution of cultural evolution. Evolutionary Anthropology 12:123-35. [AM]

Henson, R. N. A., Rugg, M. D., Shallice, T., Josephs, O. \& Dolan, R. J. (1999) Recollection and familiarity in recognition memory: An event-related functional magnetic resonance imaging study. The Journal of Neuroscience 19:3962-72. [aTS]

Hesslow, G. (2002) Conscious thought as simulation of behavior and perception. Trends in Cognitive Sciences 6(6):242-47. [aTS]

Heyes, C. M. (1998) Theory of mind in nonhuman primates. Behavioral and Brain Sciences 21:101-34. [aTS]

Holden, C. (2005) Time's up on time travel. Science 308:1110. [aTS]

Hommel, B., Müsseler, J., Ascherslebel, G. \& Prinz, W. (2001) The theory of event coding (TEC): A framework for perception and action planning. Behavioral and Brain Sciences 24(5):849-78. [JRH]

Howe, M. L. \& Courage, M. L. (1997) The emergence and early development of autobiographical memory. Psychological Review 104(3):499-523. [rTS]

Hudson, J. A. (2006) The development of future time concepts through motherchild conversation. Merrill-Palmer Quarterly Journal of Developmental Psychology 52(1):70-95. [rTS]

Hudson, J. A., Shapiro, L. R. \& Sosa, B. B. (1995) Planning in the real world: Preschool children's scripts and plans for familiar events. Child Development 66(4):984-98. [aTS]

Humphrey, N. (1976) The social function of intellect. In: Growing points in ethology, ed. P. P. G. Bateson \& R. A. Hinde. Cambridge University Press. [aTS]

Hunt, G. (1996) Manufacture and use of hook-tools by New Caledonian crows. Nature 379:249-51. [aTS]

Hunt, G. \& Gray, R. (2003) Diversification and cumulative evolution in New Caledonian crow tool manufacture. Proceedings of the Royal Society of London, Series B - Biological Sciences 270:867-74. [aTS]

Hurford, J. R. (2007) The origins of meaning. Oxford University Press. [JRH]

Hurley, S. (2006) Making sense of animals. In: Rational animals?, ed. S. Hurley \& M. Nudds, pp. 139-71. Oxford University Press. [JRH]

Ingvar, D. H. (1979) "Hyperfrontal" distribution of the cerebral grey matter flow in resting wakefulness: On the functional anatomy of the conscious state. Acta Neurologica Scandanavica 60:12-25. [RLB]

(1985) Memory of the future: An essay on the temporal organization of conscious awareness. Human Neurobiology 4:127-36. [MBa, RLB]

Janeck, A. S., Calamari, J. E., Riemann, B. C. \& Heffelfinger, S. K. (2003) Too much thinking about thinking? Metacognitive differences in obsessive-compulsive disorder. Journal of Anxiety Disorders 17:181-95. [MBr]

Jarvis, E., Gunturkun, O., Bruce, L., Csillag, A., Karten, H., Kuenzel, W., Medina, L., Paxinos, G., Perkel, D. J., Shimizu, T., Striedter, G., Wild, J. M., Ball, G. F., Dugas-Ford, J., Durand, S. E., Hough, G. E., Husband, S., Kubikova, L., Lee, D. W., Mello, C. V., Powers, A., Siang, C., Smulders, T. V., Wada, K., White, S. A., Yamamoto, K., Yu, J., Reiner, A. \& Butler, A. B. (2005) Avian brains and a new understanding of vertebrate brain evolution. Nature Reviews Neuroscience 6(2):151-59. [rTS]

Jerison, H. J. (1973) The evolution of the brain and intelligence. Academic Press. [aTS]

Jones, S., Livner, A. \& Backman, L. (2006) Patterns of prospective and retrospective memory impairment in preclinical Alzheimer's disease. Neuropsychology 20:144-52. [MBr]

Kahneman, D. \& Tversky, A. (1979) Intuitive prediction: Biases and corrective procedures. TIMS Studies in Management Science 12:313-27. [AM]

Kali, S. \& Dayan, P. (2004) Off-line replay maintains declarative memories in a model of hippocampal-neocortical interaction. Nature Neuroscience 7:28694. [aTS]

Kensinger, E. A., Garoff-Eaton, R. J. \& Schacter, D. L. (2006) Memory for specific visual details can be enhanced by negative arousing content. Journal of Memory and Language 54:99-112. [AD]

Kenward, B., Weir, A. A. S., Rutz, C. \& Kacelnik, A. (2005) Tool manufacture by naive juvenile crows. Nature 433:121. [aTS]

Kirby, K. N. (1997) Bidding on the future: Evidence against normative discounting of delayed rewards. Journal of Experimental Psychology: General 126:5470. [AM]

Klein, S. B., Cosmides, L., Tooby, J. \& Chance, S. (2002a) Decisions and the evolution of memory: Multiple systems, multiple functions. Psychological Review 109:306-29. [RLB, aTS]

Klein, S. B., Loftus, J. \& Kihlstrom, J. F. (2002b) Memory and temporal experience: The effects of episodic memory loss on an amnesic patient's ability to remember the past and imagine the future. Social Cognition 20:35379. [MBa, arTS

Koffka, K. (1935) Principles of Gestalt psychology. Routledge. [DB-K] Kolodner, J. (1993) Case-based reasoning. Morgan Kaufmann. [J-LD]

Kornell, N., Son, L. M. \& Terrace, H. S. (2007) Are monkeys metacognitive? Psychological Science 18:64-71. [SJS]

Krauth, J. (1983) Methods and problems of prediction. Neuropsychobiology 9:14753. $[\mathrm{JH}]$ 
Kroodsma, D. E. \& Momose, H. (1991) Songs of the Japanese population of the winter wren (Troglodytes troglodytes). The Condor 93:424-32. [aTS]

Lea, S. E. G. (2001) Anticipation and memory as criteria for special welfare consideration. Animal Welfare 10:(S)195-208. [aTS]

Leaver, L. A., Hopewell, L., Cadwell, C. \& Mallarky, L. (in press) Audience effects on foodcaching in grey squirrels. Animal Cognition. [aTS]

Levine, B. (2004) Autobiographical memory and the self in time: Brain lesion effects, functional neuroanatomy, and lifespan development. Brain and Cognition 55:54-68. [arTS]

Lewin, K. (1946) Behavior and development as a function of the total situation. In: Manual of child psychology, ed. L. Carmichael, pp. 791-844. Wiley. [DB-K]

Lewis, P. A. \& Miall, R. C. (2006) Remembering the time: A continuous clock. Trends in Cognitive Sciences 10:401-406. [aTS]

Locke, J. L. \& Bogin, B. (2006) Language and life history: A new perspective on the development and evolution of human language. Behavioral and Brain Sciences 29(3):259-80. [rTS]

Loftus, E. F. (1996) Eyewitness testimony. Harvard University Press. [AM]

Loftus, E. F. \& Ketcham, K. (1994) The myth of repressed memory. St. Martin's Press. [AM, aTS]

Lorenz, K. (1969) Innate bases of learning. In: On the biology of learning, ed. K. Pribram, pp. 13-91. Harcourt. [AM]

(1973/1977) Behind the mirror. Methuen. [MBr]

(1992) Die Naturwissenschaft vom Menschen. Eine Einführung in die vergleichende Verhaltensforschung. Das "Russische Manuskript," ed. A. von Cranach. Piper. [MB]

MacLeod, A. K. \& Byrne, A. (1996) Anxiety, depression, and the anticipation of future positive and negative experiences. Journal of Abnormal Psychology 105(2):286-89. [AD, rTS]

Maguire, E. A., Vargha-Khadem, F. \& Mishkin, M. (2001) The effects of bilateral hippocampal damage on fMRI regional activations and interactions during memory retrieval. Brain 124:1156-70. [rTS]

Mahon, N. E., Yarcheski, T. J. \& Yarcheski, A. (1997) Future time perspective and positive health practices in young adults: An extension. Perceptual and Motor Skills 84(3):1299-1304. [rTS]

Martin, A., Wiggs, C. L., Ungerleider, L. G. \& Haxby, J. V. (1996) Neural correlates of category-specific knowledge. Nature 379:649-52. [JRH]

Mason, M. F., Norton, M. I., Van Horn, J. D., Wegner, D. M., Grafton, S. T. \& Macrae, C. N. (2007a) Wandering minds: The default network and stimulusindependent thought. Science 315:393-95. [JO]

Mason, M. F., Norton, M. I., Van Horn, J. D., Wegner, D. M., Grafton, S. T. \& Macrae, C. N. (2007b) Response to comment on "Wandering minds: The default network and stimulus-independent thought." Science 317:43. [JO]

Mayr, E. (1982) Teleological and teleonomic: A new analysis. In: Learning, development and culture, ed. H. Plotkin, pp. 17-38. Wiley. [AM]

McClure, S. M., Laibson, D. I., Loewenstein, G. \& Cohen, J. D. (2004) The grasshopper and the ant: Separate neural systems value immediate and delayed monetary rewards. Science 306:503-07. [GA]

McFarland, C. \& Buehler, R. (1998) The impact of negative affect on autobiographical memory: The role of self-focused attention to moods. Journal of Personality and Social Psychology 75:1424-40. [AD]

McGuigan, F. \& Salmon, K. (2004) The time to talk: The influence of the timing of adult-child talk on children's event memory. Child Development 75(3):66986. [rTS]

McKenzie, T. L. B., Bird, L. R. \& Roberts, W. A. (2005) The effects of cache modification on food caching and retrieval behavior by rats. Learning and Motivation 36(2):260-78. [aTS]

Meacham, J. A. \& Singer, J. (1977) Incentive effects in prospective remembering. Journal of Psychology 97:191-97. [JRH]

Mecklinger, A., Gruenewald, C., Besson, M., Magnie, M.-N. \& Cramon, D. Y. V. (2002) Separable neuronal circuitries for manipulable and non-manipulable objects in working memory. Cerebral Cortex 12(11):1115-23. [JRH]

Menzel, E. (2005) Progress in the study of chimpanzee recall and episodic memory In: The missing link in cognition, ed. H. S. Terrace \& J. Metcalfe, pp. 188-224. Oxford University Press. [aTS]

Mercado, E., Murray, S. O., Uyeyama, R. K., Pack, A. A. \& Herman, L. M. (1998 Memory for recent actions in the bottlenosed dolphin (Tursiops truncatus): Repetition of arbitrary behaviors using an abstract rule. Animal Learning and Behavior 26(2):210-18. [aTS]

Mesoudi, A. (2007) Using the methods of social psychology to study cultural evolution. Journal of Social, Evolutionary, and Cultural Psychology 1(2):35-58. [AM]

Mesoudi, A., Whiten, A. \& Laland, K. N. (2004) Is human cultural evolution Darwinian? Evidence reviewed from the perspective of The Origin of Species. Evolution 58:1-11. [AM]

(2006) Towards a unified science of cultural evolution. Behavioral and Brain Sciences 29:329-83. [AM, rTS]

Millar, A. \& Navarick, D. J. (1984) Self-control and choice in humans: Effects of video game playing as a positive reinforcer. Learning and Motivation 15:20318. $[\mathrm{GA}]$
Mischel, H. N. \& Mischel, W. (1983) The development of children's knowledge of self-control strategies. Child Development 54:603-19. [GA, arTS]

Mischel, W., Shoda, Y. \& Rodriguez, M. L. (1989) Delay of gratification in children. Science 244:933-38. [EHMS]

Miyashita, Y. (2004) Cognitive memory: Cellular and network machineries and their top-down control. Science 306:435-40. [aTS]

Moore, C., Barresi, J. \& Thompson, C. (1998) The cognitive basis of future-oriented prosocial behavior. Social Development 7(2):198-218. [aTS]

Moore, C. \& Lemmon, K., eds. (2001) The self in time: Developmental perspectives. Erlbaum. [KN, rTS $]$

Mulcahy, N. J. \& Call, J. (2006) Apes save tools for future use. Science 312:103840. [MBa, JRH, MO, EHMS, arTS]

Mulcahy, N. J., Call, J. \& Dunbar, R. I. M. (2005) Gorillas (Gorilla gorilla) and orangutans (Pongo pygmaeus) encode relevant problem features in a tool-using task. Journal of Comparative Psychology 119:23-32. [aTS]

Naqshbandi, M. \& Roberts, W. A. (2006) Anticipation of future events in squirrel monkeys (Saimiri sciureus) and rats (Rattus norvegicus): Tests of the BischofKöhler hypothesis. Journal of Comparative Psychology 120:345-57. [MO, rTS]

Nauta, W. J. H. (1971) The problem of the frontal lobe: A reinterpretation. Journal of Psychiatric Research 8:167-87. [BM]

Navarick, D. J. (1982) Negative reinforcement and choice in humans. Learning and Motivation 13:361-77. [GA]

Nelson, K. (1993a) Explaining the emergence of autobiographical memory in early childhood. In: Theories of memory, ed. A. Collins, M. Conway, S. Gathercole \& P. Morris. Erlbaum. [KN]

(1993b) The psychological and social origins of autobiographical memory. Psychological Science 4:1-8. [KN]

(1996) Language in cognitive development: The emergence of the mediated mind. Cambridge University Press. [KN]

(2001) Language and the self: From the "Experiencing I" to the "Continuing Me." In: The self in time: Developmental issues, ed. C. Moore \& K. Lemmon, pp. 15-34. Erlbaum. [KN]

(2005) Evolution and development of human memory systems. In: Origins of the social mind: Evolutionary psychology and child development, ed. B. Ellis \& D. Bjorklund, pp. 319-345. Guilford. [KN]

Nelson, K. D. \& Fivush, R. (2004) The emergence of autobiographical memory: A social cultural developmental theory. Psychological Review 111(2):486 511. [KN, rTS $]$

Newby-Clark, I. R. \& Ross, M. (2003) Conceiving the past and future. Personality and Social Psychology Bulletin 29:807-18. [AD]

Nielsen, M., Collier-Baker, E., Davis, J. \& Suddendorf, T. (2005) Imitation recognition in a captive chimpanzee (Pan Troglodytes). Animal Cognition 8:31-36. [aTS]

Nielsen, M., Suddendorf, T. \& Slaughter, V. (2006) Self-recognition beyond the face. Child Development 77:176-85. [aTS]

Nimchinsky, E. A., Vogt, B. A., Morrison, J. H. \& Hof, P. R. (1995) Spindle neurons of the human anterior cingulate cortex. Journal of Comparative Neurology $355: 27-37 . \quad[\mathrm{MBr}]$

Noë, A. (2004) Action in perception. MIT Press. [JRH]

Odling-Smee, F. J., Laland, K. K. \& Feldman, M. W. L. (2003) Niche construction: The neglected process in evolution. Princeton University Press. [aTS]

O'Doherty, J. P. (2004) Reward representations and reward-related learning in the human brain: Insights from neuroimaging. Current Opinion in Neurobiology 14:769-76. [aTS]

O'Doherty, J. P., Deichmann, R., Critchley, H. D. \& Dolan, R. J. (2002) Neural responses during anticipation of a primary taste reward. Neuron 33(5):81526. [aTS]

Okuda, J., Fujii, T., Ohtake, H., Tsukiura, T., Tanji, K., Suzuki, K., Kawashima, R., Fukuda, H., Itoh, M. \& Yamadori, A. (2003) Thinking of the future and past: The roles of the frontal pole and the medial temporal lobes. Neuroimage 19:1369-80. [RLB, VAC, JO, DLS, arTS]

Okuda, J., Fujii, T., Ohtake, H., Tsukiura, T., Yamadori, A., Frith, C. D. \& Burgess, P. W. (2007) Differential involvement of regions of rostral prefrontal cortex (Brodmann area 10) in time- and event-based prospective memory. International Journal of Psychophysiology 64:233-46. [JO]

O’Reilly, R. C. \& McClelland, J. L. (1994) Hippocampal conjunctive encoding, storage, and recall: Avoiding a trade-off. Hippocampus 4:661-82. [VAC]

O'Reilly, R. C. \& Rudy, J. W. (2001) Conjunctive representations in learning and memory: Principles of cortical and hippocampal function. Psychological Review 108:311-45. [VAC]

Osvath, M. \& Gärdenfors, P. (2005) Oldowan culture and the evolution of anticipatory cognition. Lund University Cognitive Studies 122:1-16. [MO, arTS]

Owen, A. M. (2003) HERA today, gone tomorrow? Trends in Cognitive Sciences $7(9): 383-84 . \quad[\mathrm{rTS}]$

Patterson, F. \& Cohen, R. H. (1994) Self-recognition and self-awareness in lowland gorillas. In: Self-awareness in animal and humans, ed. S. T. Parker, R. W. Mitchell \& M. L. Boccia, pp. 273-90. Cambridge University Press. [aTS]

Perner, J. (1991) Understanding the representational mind. MIT Press. [aTS] 
(2000) Memory and theory of mind. In: The Oxford handbook of memory, ed. E. Tulving \& F. I. M. Craik, pp. 297-312. Oxford University Press. [rTS]

Perner, J. \& Ruffman, T. (1995) Episodic memory and autonoetic consciousness: Developmental evidence and a theory of childhood amnesia. Journal of Experimental Child Psychology 59(3):516-48. [rTS]

Pica, P., Lemer, C., Izard, V. \& Dehaene, S. (2004) Exact and approximate arithmetic in an Amazonian indigene group. Science 306:499-503. [SJS]

Pinker, S. (2003) Language as an adaptation to the cognitive niche. In: Language evolution, ed. M. H. Christiansen \& S. Kirby, pp. 16-37. Oxford University Press. [aTS]

Piolino, P., Desgranges, B., Belliard, S., Matuszewski, V., Lalevee, C., de la Sayette, V. \& Eustache, F. (2003) Autobiographical memory and autonoetic consciousness: Triple dissociation in neurodegenerative diseases. Brain 126:220319. $[\mathrm{MBr}]$

Piolino, P., Hisland, M., Ruffeveille, I., Matuszewski, V., Jambaque, I. \& Eustache, F. (2007) Do school-age children remember or know the personal past? Consciousness and Cognition 16(1):84-101. [rTS]

Plummer, T. (2004) Flaked stones and old bones: Biological and cultural evolution at the dawn of the dawn of technology. Yearbook of Physical Anthropology 47:118-64. [MO]

Povinelli, D. J. (2000) Folk physics for apes. Oxford University Press. [aTS]

Povinelli, D. J. \& Vonk, J. (2003) Chimpanzee minds: Suspiciously human? Trends in Cognitive Sciences 7:157-60. [aTS]

Prabhakaran, V., Narayanan, K., Zhao, Z. \& Gabrieli, J. D. E. (2000) Integration of diverse information in working memory within the frontal lobe. Nature Neuroscience 3:85-90. [DLS]

Premack, D. \& Woodruff, G. (1978) Does the chimpanzee have a theory of mind? Behavioral and Brain Sciences 1:515-26. [arTS]

Quiroga, R. Q., Reddy, L., Kreiman, G., Koch, C. \& Fried, I. (2005) Invariant visual representation by single neurons in the human brain. Nature 435:1102107. [VAC]

Raby, C. R., Alexis, D. M., Dickinson, A. \& Clayton, N. S. (2007) Planning for the future by western scrub-jays. Nature 445(7130):919-21. [MBa, VAC, JRH, MO, CR, DLS, EHMS, SJS, rTS, ET]

Ramseyer, A., Pelé, M., Dufour, V., Chauvin, C. \& Thierry, B. (2006) Accepting loss: The temporal limits of reciprocity in brown capuchin monkeys. Proceedings of the Royal Society B 273:179-84. [EHMS]

Reebs, S. G. \& Lague, M. (2000) Daily food-anticipatory activity in golden shiners A test of endogenous timing mechanisms. Physiology and Behavior 70:3543. [MBa]

Reese, E. (2002) Social factors in the development of autobiographical memory: The state of the art. Social Development 11(1):124-42. [rTS]

Retherford, R. D. \& Sewell, W. H. (1989) How intelligence affects fertility. Intelligence 13:169-85. [GA]

Rhue, J. W. \& Lynn, S. J. (1987) Fantasy proneness: The ability to hallucinate "as real as real." British Journal of Experimental and Clinical Hypnosis 4:173-80. [GA]

Richerson, P. J. \& Boyd, R. (2005) Not by genes alone. University of Chicago Press. [AM]

Rindos, D. (1985) Darwinian selection, symbolic variation, and the evolution of culture. Current Anthropology 26:65-88. [AM]

Roberts, K. P. \& Blades, M., eds. (2000) Children's Source Monitoring. Erlbaum. [KN]

Roberts, W. A. (2002) Are animals stuck in time? Psychological Bulletin 128(3):473-89. [SJS, arTS]

(2006) Animal memory: Episodic-like memory in rats. Current Biology 16(15):R601-R603. [rTS]

Roediger, H. L., III \& McDermott, K. B. (1995) Creating false memories remembering words not presented in lists. Journal of Experimental Psychology: Learning Memory and Cognition 21:803-14. [aTS]

Rolls, E. T. \& Kesner, R. P. (2006) A computational theory of hippocampal function, and empirical tests of the theory. Progress in Neurobiology 79:1-48. [VAC]

Rubin, D. C. \& Schulkind, M. D. (1997) Distribution of important and word-cued autobiographical memories in 20-, 35-, and 70-year-old adults. Psychology and Aging 12(3):524-35. [aTS]

Sacks, O. (1985) The man who mistook his wife for a hat. Picador. [MBr]

Sanna, L. J. (2000) Mental simulation, affect, and personality: A conceptual framework. Current Directions in Psychological Science 9:168-73. [AD]

Schacter, D. L. (1996) Searching for memory: The brain, the mind, and the past. Basic Books. [aTS]

(1999) The seven sins of memory - Insights from psychology and cognitive neuroscience. American Psychologist 54(3):182-203. [rTS]

Schacter, D. L. \& Addis, D. R. (2007a) The cognitive neuroscience of constructive memory: Remembering the past and imagining the future. Philosophical Transactions of the Royal Society (B) Biological Sciences. 362(1481):77386. [RLB, AD, DLS, rTS ]

(2007b) The ghosts of past and future. Nature 445(7123):27. [DLS, rTS, ET]

Schank, R. C. \& Abelson, R. (1977) Scripts, plans, goals, and understanding. Erlbaum. [JRH]
Scheumann, M. \& Call, J. (2006) Sumatran orangutans and a yellow-cheeked crested gibbon know what is where. International Journal of Primatology 27(2):575-602. [aTS]

Schoenemann, P. T., Sheenan, M. J. \& Glotzer, L. D. (2005) Prefrontal white matter volume is disproportionately larger in humans than in other primates. Nature Neuroscience 8:242-52. [aTS]

Schultz, W. (2006) Behavioral theories and the neurophysiology of reward. Annual Review of Psychology 57:87-115. [aTS]

Schwartz, B. L. \& Evans, S. (2001) Episodic memory in primates. American Journal of Primatology 55(2):71-85. [aTS]

Schwartz, B. L., Colon, M. R., Sanchez, I. C., Rodriguez, I. A. \& Evans, S. (2002) Singletrial learning of "what" and "who" information in a gorilla (Gorilla gorilla gorilla): Implications for episodic memory. Animal Cognition 5(2):85-90. [aTS]

Schwartz, B. L., Hoffman, M. L. \& Evans, S. (2005) Episodic-like memory in a gorilla: A review and new findings. Learning and Motivation 36(2):226-44. [aTS]

Schwartz, B. L., Meissner, C. A., Hoffman, M. L., Evans, S. \& Frazier, L. D. (2004) Event memory and misinformation effects in a gorilla (Gorilla gorilla gorilla). Animal Cognition 7:93-100. [JRH]

Semendeferi, K., Armstrong, E., Schleicher, A., Zilles, K. \& van Hoesen, G. W. (1998) Limbic frontal cortex in hominoids: A comparative study of area 13. American Journal of Physical Anthropology 106:129-55. [aTS]

(2001) Prefrontal cortex in humans and apes: A comparative study of area 10. American Journal of Physical Anthropology 114:224-41. [aTS]

Semendeferi, K., Damasio, H. \& Frank, R. (1997) The evolution of the frontal lobes: A volumetric analysis based on three-dimensional reconstructions of magnetic resonance scans of human and ape brains. Journal of Human Evolution $32: 375-88 . \quad$ [aTS]

Seyfarth, R. M., Cheney, D. L. \& Bergman, T. J. (2005) Primate social cognition and the origins of language. Trends in Cognitive Sciences 9:264-66. [aTS]

Shapiro, M. A. \& Fox, J. R. (2002) The role of typical and atypical events in story memory. Human Communication Research 28(1):109-35. [J-LD]

Shefrin, H. M. \& Thaler, R. H. (1988) The behavioral life-cycle hypothesis. Economic Inquiry 26:609-43. [GA]

Shettleworth, S. J. (1998) Cognition, evolution, and behavior. Oxford University Press. [SJS]

(2007) Animal behaviour - Planning for breakfast. Nature 445(7130):82526. [CR, SJS, rTS]

Shettleworth, S. J. \& Sutton, J. E. (2006) Do animals know what they know? In: Rational animals?, ed. S. Hurley \& M. Nudds, pp. 235-46. Oxford University Press. [aTS]

Simon, J. L. (1995) Interpersonal allocation continuous with intertemporal allocation: Binding commitments, pledges, and bequests. Rationality and Society 7:367-430. [GA]

Simonton, D. K. (1999) Creativity as blind variation and selective retention: Is the creative process Darwinian? Psychological Inquiry 10:309-28. [AM]

Smith, C. C. \& Reichman, O. J. (1984) The evolution of food caching by birds and mammals. Annual Review of Ecology and Systematics 15:329-51. [aTS]

Smith, J. D., Shields, W. E. \& Washburn, D. A. (2003) The comparative psychology of uncertainty monitoring and metacognition. Behavioral and Brain Sciences 26(3):317. [SJS, aTS]

Smith, J. D. \& Washburn, D. A. (2005) Uncertainty monitoring and metacognition by animals. Current Directions in Psychological Science 14:19-24. [SJS]

Son, L. K. \& Kornell, N. (2005) Metaconfidence judgements in rhesus macaques: Explicit versus implicit mechanisms. In: The missing link in cognition, ed. H. S. Terrace \& J. Metcalfe, pp. 296-320. Oxford University Press. [aTS]

Spreng, R. N. \& Levine, B. (2006) The temporal distribution of past and future autobiographical events across the lifespan. Memory and Cognition 34:164451. [ET, aTS $]$

Squire, L. R. (1992) Declarative and nondeclarative memory: Multiple brain systems supporting learning and memory. Journal of Cognitive Neuroscience $4(3): 232-43 . \quad$ [aTS $]$

Squire, L. R. \& Zola, S. M. (1998) Episodic memory, semantic memory, and amnesia. Hippocampus 8:205-11. [MBa]

Stangor, C. \& McMillan, D. (1992) Memory for expectancy-congruent and expectancy-incongruent information: A review of the social and social developmental literatures. Psychological Bulletin 111(1):42-61. [J-LD]

Steele, M. A., Turner, G., Smallwood, P. D., Wolff, J. O. \& Radillo, J. (2001) Cache management by small mammals: Experimental evidence for the significance of acorn-embryo excision. Journal of Mammalogy 82(1):35-42. [aTS]

Sterelny, K. (2003) Thought in a hostile world. Blackwell. [aTS]

Suarez, S. \& Gallup, G. G. (1981) Self recognition in chimpanzees and orangutans, but not gorillas. Journal of Human Evolution 10:175-88. [aTS]

Suddendorf, T. (1994) Discovery of the fourth dimension: Mental time travel and human evolution. Unpublished Masters thesis, University of Waikato, Hamilton. Available at: http://cogprints.org/729/ [arTS]

(1999) The rise of the metamind. In: The descent of mind: Psychological perspectives on hominid evolution, ed. M. C. Corballis \& S. E. G. ea, pp. 218-60. Oxford University Press. Available at: http://cogprints.org/732/ [arTS] 
(2004) How primatology can inform us about the evolution of the human mind. Australian Psychologist 39(3):180-87. [arTS]

(2006) Foresight and evolution of the human mind. Science 312(5776):10061007. [EHMS, arTS]

Suddendorf, T. \& Busby, J. (2003a) Like it or not? The mental time travel debate. Trends in Cognitive Sciences 7:437-38. [aTS]

(2003b) Mental time travel in animals? Trends in Cognitive Sciences 7(9):391396 [arTS]

(2005) Making decisions with the future in mind: Developmental and comparative identification of mental time travel. Learning and Motivation 36(2):11025. [CMA, arTS]

Suddendorf, T. \& Corballis, M. C. (1997) Mental time travel and the evolution of the human mind. Genetic Social and General Psychology Monographs 123(2):133-67. Available at: http://cogprints.org/725/ [RLB, DLS, CR, arTS]

Suddendorf, T. \& Whiten, A. (2001) Mental evolution and development: Evidence for secondary representation in children, great apes and other animals. Psychological Bulletin 127(5):629-50. [arTS]

(2003) Reinterpreting the mentality of apes. In: From mating to mentality: Evaluating evolutionary psychology, ed. K. Sterelny \& J. Fitness, pp 173-96. Psychology Press. [aTS]

Sutton, J. E. \& Shettleworth, S. J. (in press) Memory without awareness: Pigeons fail to show metamemory in matching to sample. Journal of Experimental Psychology: Animal Behavior Processes. [SJS]

Suzuki, W. A. \& Amaral, D. G. (1994) Perirhinal and parahippocampal cortices of the macaque monkey: Cortical afferents. Journal of Comparative Neurology 350:497-533. [VAC]

Szagun, G. (1978) On the frequency of use of tenses in English and German children's spontaneous speech. Child Development 49:898-901. [aTS]

Szpunar, K. K., Watson, J. M. \& McDermott, K. B. (2007) Neural substrates of envisioning the future. Proceedings of the National Academy of Sciences USA 104(2):642-47. [RLB, JO, DLS, rTS, ET]

Talarico, J. M., LaBar, K. S. \& Rubin, D. C. (2004) Emotional intensity predicts autobiographical memory experience. Memory and Cognition 32:1118-32. [AD]

Tanaka, S. C., Doya, K., Okada, G., Ueda, K., Okamoto, Y. \& Yamawaki, S. (2004) Prediction of immediate and future rewards differentially recruits cortico-basal ganglia loops. Nature Neuroscience 7:887-93. [aTS]

Tannen, D. (1984) Conversational style - Analyzing talk among friends. Ablex. [J-LD]

Taylor, S. E. \& Brown, J. D. (1988) Illusion and well-being: A social psychological perspective on mental health. Psychological Bulletin 103:193210. [AD]

Terrace, H. S. \& Metcalfe, J., eds. (2005) The missing link in cognition: Origins of self-reflective consciousness. Oxford University Press. [SJS]

Thieme, H. (1997) Lower Palaeolithic hunting spears from Germany. Nature 385(6619):807-10. [aTS]

Thompson, C., Barresi, J. \& Moore, C. (1997) The development of future-oriented prudence and altruism in preschoolers. Cognitive Development 12:199_ 212. [aTS]

Thompson, N. S. (1969) Individual identification and temporal patterning in the cawing of common crows. Communications in Behavioral Biology 4:29-33. [aTS]

Thorpe, C. M., Jacova, C. \& Wilkie, D. M. (2004) Some pitfalls in measuring memory in animals. Neuroscience and Biobehavioral Reviews 28(7):711-18. [aTS]

Tolman, E. C. (1939) Prediction of vicarious trial and error by means of the schematic sowbug. Psychological Review 46:318-36. [GA]

Tomasello, M., Call, J. \& Hare, B. (2003) Chimpanzees understand psychological states - The question is which ones and to what extent. Trends in Cognitive Sciences 7:53-156. [aTS]

Tomasello, M., Carpenter, M., Call, J., Behne, T. \& Moll, H. (2005) Understanding and sharing intentions: The origins of cultural cognition. Behavioral and Brain Sciences 28:675-735. [aTS]

Tooby, J. \& DeVore, I. (1987) The reconstruction of hominid behavioral evolution through strategic modelling. In: The evolution of human behavior: Primate models, ed. W. Kinzey, pp. 183-238. State University of New York Press. [aTS]

Toth, N. (1985) The Oldowan reassessed: A close look at early stone artefacts. Journal of Archeological Science 12:101-20. [MO]

Tulving, E. (1972) Episodic and semantic memory. In: Organization of memory, ed. E. Tulving \& W. Donaldson, pp. 382-403. Academic Press. [WJF, CR, aTS] (1983) Elements of episodic memory. Clarendon Press. [KN, JO, rTS] (1984) Précis of Elements of episodic memory. Behavioral and Brain Sciences $7(2): 223-68 . \quad[\mathrm{MB}, \mathrm{WJF}, \mathrm{aTS}]$

(1985) Memory and consciousness. Canadian Journal of Psychology 26:112. [RLB, arTS, ET]

(1993) What is episodic memory? Current Directions in Psychological Science 2(3):67-70. [KN, JO]

(2002a) Chronesthesia: Awareness of subjective time. In: Principles of frontal lobe functions, ed. D. T. Stuss \& R. C. Knight, pp. 311-25. Oxford University Press. [ET] (2002b) Episodic memory: From mind to brain. Annual Review of Psychology 53:1-25. [WJF]

(2005) Episodic memory and autonoesis: Uniquely human? In: The missing link in cognition: Origins of self-reflective consciousness, ed. H. S. Terrace \& J. Metcalfe, pp. 3-56. Oxford University

Press. [JRH, KN, JO, arTS]

Uncapher, M. R. \& Rugg, M. D. (2005) Encoding and the durability of episodic memory: A functional magnetic resonance imaging study. Journal of Neuroscience 25:7260-67. [VAC]

Uylings, H. B. M. (1990) The prefrontal cortex: Its structure, function, and pathology. Elsevier. [aTS]

Vallortigara, G. \& Rogers, L. J. (2005) Survival with an asymmetrical brain: Advantages and disadvantages of cerebral lateralization. Behavioral and Brain Sciences 28(4):575-89. [rTS]

Viskontas, I. V. (2006) The contributions of medial temporal lobe subregions to declarative memory processes. Unpublished doctoral dissertation, Department of Psychology, University of California, Los Angeles. [VAC]

Viskontas, I. V., Knowlton, B. J., Steinmetz, P. N. \& Fried, I. (2006) Differences in mnemonic processing by neurons in the human hippocampus and parahippocampal regions. Journal of Cognitive Neuroscience 18:1654-62. [VAC]

Vogeley, K. \& Kupke, C. (2007) Disturbances of time consciousness from a phenomenological and a neuroscientific perspective. Schizophrenia Bulletin 33:157-65. [MBr]

Weinstein, N. D. (1980) Unrealistic optimism about future life events. Journal of Personality and Social Psychology 39:806-20. [AD]

Welch-Ross, M. K. (1995) An integrative model of the development of autobiographical memory. Developmental Review 15(3):338-65. [rTS]

Wellman, H. M., Cross, D. \& Watson, J. (2001) Meta-analysis of theory-of-mind development: The truth about false belief. Child Development 72:655-84. [aTS]

Wheeler, M. A., Stuss, D. T. \& Tulving, E. (1997) Toward a theory of episodic memory: The frontal lobes and autonoetic consciousness. Psychological Bulletin 121(3):331-54. [arTS]

Whiten, A. (1998) Imitation of the sequential structure of actions by chimpanzees (Pan troglodytes). Journal of Comparative Psychology 112:270-81. [aTS]

(2001) Folk physics for apes: The chimpanzee's theory of how the world works. Nature 409(6817):133. [aTS]

Whiten, A. \& Byrne, R. W. (1988) Tactical deception in primates. Behavioral and Brain Sciences 11:233-73. [aTS]

Whiten, A. \& Suddendorf, T. (2001) Meta-representation and secondary representation. Trends in Cognitive Sciences 5:378. [aTS]

(in press) Great ape cognition and the evolutionary roots of human imagination. In: Imaginative minds, ed. I. Roth. Oxford University Press. [arTS]

Williams, J. H. G., Whiten, A., Suddendorf, T. \& Perrett, D. I. (2001) Imitation, mirror neurons and autism. Neuroscience and Biobehavioral Reviews 25(4):287-95. [rTS]

Williams, J. M. G., Ellis, N. C., Tyers, C., Healy, H., Rose, G. \& MacLeod, A. K. (1996) The specificity of autobiographical memory and imageability of the future Memory and Cognition 24:116-25. [aTS]

Wilson, M. A. \& McNaughton, B. L. (1994) Reactivation of Hippocampal Ensemble Memories During Sleep. Science 265(5172):676-79. [aTS]

Wilson, T. D. \& Gilbert, D. T. (2005) Affective forecasting: Knowing what to want. Current Directions in Psychological Science 14:131-34. [aTS]

Woll, S. \& Graesser, A. (1982) Memory discrimination for information typical and atypical of person schemata. Social Cognition 1:287-310. [J-LD]

Wood, B. \& Collard, M. (1999) Anthropology - The human genus. Science 284(5411):65-71. [aTS]

Woodruff, C. C., Johnson, J. D., Uncapher, M. R. \& Rugg, M. D. (2005) Contentspecificity of the neural correlates of recollection. Neuropsychologia 43:102232. [VAC

Wynne, C. D. L. (2007) What are animals? Why anthropomorphism is still not a scientific approach to behavior. Comparative Cognition and Behavior Reviews 2:125-35. [SJS]

Yonelinas, A. P. (1994) Receiver-operating characteristics in recognition memory evidence for a duel-process model. Journal of Experimental Psychology Learning, Memory, and Cognition 20:1341-54. [MJE]

Yonelinas, A. P., Kroll, N. E. A., Dobbins, I., Lazzara, M. \& Knight, R. T. (1998) Recollection and familiarity deficits in amnesia: Convergence of remember/ know, process dissociation and receiver operating characteristic data. Neuropsychology 12:323-39. [MJE]

Zentall, T. R. (2005) Animals may not be stuck in time. Learning and Motivation $36: 208-25 . \quad[\mathrm{WJF}]$

(2006) Mental time travel in animals: A challenging question. Behavioural Processes 72:173-83. [arTS]

Zentall, T. R., Clement, T. S., Bhatt, R. S. \& Allen, J. (2001) Episodic-like memory in pigeons. Psychonomic Bulletin and Review 8(4):685-90. [aTS]

Zimbardo, P. G. \& Boyd, J. N. (1999) Putting time in perspective: A valid, reliable individual-differences metric. Journal of Personality and Social Psychology $77(6): 1271-88 . \quad[\mathrm{rTS}]$ 